\title{
Oblique boundary value problems for augmented Hessian equations I
}

\author{
Feida Jiang $^{1}$ - Neil S. Trudinger ${ }^{2,3}{ }_{\mathbb{D}}$
}

Received: 12 April 2018 / Accepted: 2 May 2018 / Published online: 21 May 2018

(C) The Author(s) 2018

\begin{abstract}
In this paper, we study global regularity for oblique boundary value problems of augmented Hessian equations for a class of general operators. By assuming a natural convexity condition of the domain together with appropriate convexity conditions on the matrix function in the augmented Hessian, we develop a global theory for classical elliptic solutions by establishing global a priori derivative estimates up to second order. Besides the known applications for Monge-Ampère type operators in optimal transportation and geometric optics, the general theory here embraces Neumann problems arising from prescribed mean curvature problems in conformal geometry as well as general oblique boundary value problems for augmented $k$-Hessian, Hessian quotient equations and certain degenerate equations.
\end{abstract}

Communicated by S. K. Jain.

Research supported by National Natural Science Foundation of China (No. 11771214), Australian Research Council (No. DP1094303).

Neil S. Trudinger

Neil.Trudinger@anu.edu.au

Feida Jiang

jfd2001@163.com

1 College of Mathematics and Statistics, Nanjing University of Information Science and Technology, Nanjing 210044, People's Republic of China

2 Centre for Mathematics and Its Applications, The Australian National University, Canberra, ACT 0200, Australia

3 School of Mathematics and Applied Statistics, University of Wollongong, Wollongong, NSW 2522, Australia 
Keywords Oblique boundary value problems - Augmented Hessian equations • Second derivative estimates · Gradient estimates

Mathematics Subject Classification $35 \mathrm{~J} 60 \cdot 35 \mathrm{~J} 25$

\section{Introduction}

In this paper we develop the essentials of a general theory of classical solutions of oblique boundary value problems for certain types of fully nonlinear elliptic partial differential equations, which we describe as augmented Hessian equations. Such problems arise in various applications, notably to optimal transportation, geometric optics and conformal geometry and our critical domain and augmenting matrix convexity notions are adapted from those introduced in [31,40,45] for regularity in optimal transportation. Our main concern here will be with semilinear boundary conditions but we will also cover the nonlinear case for appropriate subclasses of our general operators. The classical solvability of the Neumann problem for the MongeAmpère equation was proved by Lions et al. [27]. Not only was the approach in [27] special for the Neumann problem, but it follows from the fundamental example of Pogorelov [32] that the result cannot be extended to general linear oblique boundary value problems $[47,52]$. On the other hand, the classical Dirichlet problem for basic Hessian equations has been well studied in the wake of fundamental papers by Caffarelli et al. [1] and Ivochkina [9], with further key developments by several authors, including Krylov [20] and related papers and Trudinger [38]; (see also [8] for a recent account of the resultant theory under fairly general conditions).

Our main concerns in this paper are second derivative estimates under natural "strict regularity" conditions on the augmenting matrices, together with accompanying gradient and Hölder estimates, which then lead to classical existence theorems. Our theory embraces a wide class of examples which we also present as well as a key application to semilinear Neumann problems arising in conformal geometry, where remarkably our adaptation of optimal transportation domain convexity from $[40,45]$ enables us to replace the rather strong umbilic boundary condition for second derivative bounds, assumed in previous work [3,19], by more general natural convexity conditions, (in the prescribed positive mean curvature case). In ensuing papers we consider extensions to weaker matrix convexity conditions as well as the regularity of weak solutions and the sharpness of our domain convexity conditions. Extensions to the Dirichlet problem for our general class of equations are treated in [14]. Overall this paper provides a comprehensive framework for studying oblique boundary value problems for a large class of fully nonlinear equations, which embraces the Monge-Ampère type case in Section 4 in [16] as a special example.

Specifically we study augmented Hessian partial differential equations of the form,

$$
\mathcal{F}[u]:=F\left[D^{2} u-A(\cdot, u, D u)\right]=B(\cdot, u, D u), \quad \text { in } \Omega,
$$


subject to boundary conditions

$$
\mathcal{G}[u]:=G(\cdot, u, D u)=0, \quad \text { on } \partial \Omega,
$$

where $\Omega$ is a bounded domain in $n$ dimensional Euclidean space $\mathbb{R}^{n}$ with smooth boundary, $D u$ and $D^{2} u$ denote the gradient vector and the Hessian matrix of the solution $u \in C^{2}(\Omega), A$ is a $n \times n$ symmetric matrix function defined on $\Omega \times \mathbb{R} \times \mathbb{R}^{n}$, $B$ is a scalar valued function on $\Omega \times \mathbb{R} \times \mathbb{R}^{n}$ and $G$ is a scalar valued function defined on $\partial \Omega \times \mathbb{R} \times \mathbb{R}^{n}$. We use $x, z, p, r$ to denote the points in $\Omega, \mathbb{R}, \mathbb{R}^{n}$ and $\mathbb{R}^{n \times n}$ respectively. For $G \in C^{1}\left(\partial \Omega \times \mathbb{R} \times \mathbb{R}^{n}\right)$, the boundary condition (1.2) is said to be oblique, with respect to $u \in C^{1}(\bar{\Omega})$, if

$$
G_{p}(\cdot, u, D u) \cdot v \geq \beta_{0}, \quad \text { on } \partial \Omega,
$$

where $v$ is the unit inner normal vector field on $\partial \Omega$ and $\beta_{0}$ is a positive constant. If $G_{p} \cdot v>0$ on all of $\partial \Omega \times \mathbb{R} \times \mathbb{R}^{n}$, we will simply refer to $G$ (or $\mathcal{G}$ ) as oblique. In the context, we shall use either $\mathcal{F}$ or $F$ to denote the general operator in (1.1), and either $\mathcal{G}$ or $G$ to denote the boundary operator in (1.2).

Letting $\mathbb{S}^{n}$ denote the linear space of $n \times n$ symmetric matrices, the function $F$ in (1.1) is defined on an open, convex cone $\Gamma$ in $\mathbb{S}^{n}$, with vertex at 0 , containing the positive cone $K^{+}$. In order to consider $F$ in a very general setting, we assume that $F \in C^{2}(\Gamma)$ satisfies a subset of the following properties.

F1 $F$ is strictly increasing in $\Gamma$, namely

$$
F_{r}:=F_{r_{i j}}=\left\{\frac{\partial F}{\partial r_{i j}}\right\}>0 \text {, in } \Gamma .
$$

F2 $F$ is concave in $\Gamma$, namely

$$
\frac{\partial^{2} F}{\partial r_{i j} \partial r_{k l}} \eta_{i j} \eta_{k l} \leq 0 \text {, in } \Gamma,
$$

for all symmetric matrices $\left\{\eta_{i j}\right\} \in \mathbb{S}^{n}$.

F3 $F(\Gamma)=\left(a_{0}, \infty\right)$ for a constant $a_{0} \geq-\infty$ with

$$
\sup _{r_{0} \in \partial \Gamma} \limsup _{r \rightarrow r_{0}} F(r) \leq a_{0}
$$

F4 $\quad F(t r) \rightarrow \infty$ as $t \rightarrow \infty$, for all $r \in \Gamma$.

F5 For given constants $a, b$ satisfying $a_{0}<a<b$, there exists a constant $\delta_{0}>0$ such that $\mathscr{T}(r):=\operatorname{trace}\left(F_{r}\right) \geq \delta_{0}$ if $a<F(r)<b$.

F5 $^{+} \quad \mathscr{T}(r) \rightarrow \infty$ uniformly for $a \leq F(r) \leq b$ as $|r| \rightarrow \infty$.

We say an operator $\mathcal{F}$ satisfies the above properties, if the corresponding function $F$ satisfies them. Note that we can take the constant $a_{0}$ in F2 and F5 to be 0 or $-\infty$. When $F$ is given as a symmetric function $f$ of the eigenvalues $\lambda_{1}, \ldots, \lambda_{n}$ of the matrix 
$r$, with $\Gamma$ closed under orthogonal transformations, we will refer to $\mathcal{F}$ as orthogonally invariant. In this case the above conditions are modelled on the conditions introduced for the study of the Dirichlet problem for the basic Hessian equations, with $A=0$, by Caffarelli et al. [1] and Trudinger [38]. The standard nonlinear operators satisfying the above properties are the $k$-Hessian operators, $F_{k}=\left(S_{k}\right)^{\frac{1}{k}}, k=2, \ldots, n$, which satisfy $\mathrm{F} 1-\mathrm{F} 4, \mathrm{~F}^{+}$on $\Gamma_{k}$ with $a_{0}=0$, and their quotients $F_{k, l}=\left(\frac{S_{k}}{S_{l}}\right)^{\frac{1}{k-l}}, n \geq k>l \geq 1$, which satisfy F1-F5 on $\Gamma_{k}$ with $a_{0}=0$, where $S_{k}$ denotes the $k$-th order elementary symmetric function defined by

$$
S_{k}[r]:=S_{k}(\lambda(r))=\sum_{1 \leq i_{1}<\cdots<i_{k} \leq n} \lambda_{i_{1}} \ldots \lambda_{i_{k}}, k=1, \ldots, n,
$$

and $\Gamma_{k}$ denotes the cone defined by

$$
\Gamma_{k}=\left\{r \in \mathbb{S}^{n} \mid S_{j}[r]>0, \forall j=1, \ldots, k\right\} .
$$

As usual we set $F_{0}=1$, so that we can also write the standard $k$-Hessian $F_{k}$ as the quotient $F_{k, 0}$. It turns out that the proofs of our results and their underlying ideas are essentially just as complicated for these special cases as for the general situation so that a reader will not miss the main features of our techniques by restricting attention to them. More generally when $a_{0}=0$ and $F$ is positive homogeneous of degree one, then properties F1, F2, F3 imply F4 and F5, with $a=0$ and $b=\infty$. Clearly F4 is obvious and to show F5 we have by the concavity F2, for a positive constant $\mu$ and $r \in \Gamma$,

$$
\mathscr{T}(r) \geq \frac{1}{\mu}\left\{F(\mu I)-F(r)+r \cdot F_{r}\right\}=F(I)>0,
$$

where the equality follows from the homogeneity, which implies $r \cdot F_{r}=F(r)$. (Clearly it is enough to take $\mu=1$ here but it is convenient to use a general $\mu$ for later use).

We also note in general that F2 and F3 imply, for $r \in \Gamma$ and finite $a_{0}$,

$$
0 \leq r \cdot F_{r} \leq F(r)-a_{0}
$$

By the concavity $\mathrm{F} 2$, we have, for $t>0$,

$$
F(t r)-F(r) \leq(t-1) r \cdot F_{r},
$$

from which (1.10) follows by taking $t$ sufficiently large for the first inequality and sufficiently small for the second. If $a_{0}=-\infty$, then (1.10) clearly holds if also F4 is satisfied, (or more generally lim inf $F(t r)>-\infty$ as $t \rightarrow \infty$ ). From (1.9) and (1.10), we then obtain for $F(r) \leq b$,

$$
\mathscr{T}(r) \geq \frac{1}{\mu}\{F(\mu I)-b\} \geq \delta_{0}>0,
$$


for some constant $\delta_{0}$, depending on $F$ and $b$, by taking $\mu$ sufficiently large, so that condition F5, with $a=a_{0}$, is itself a consequence of F2 and F4. Moreover, when $a_{0}$ is finite, both F4 and F5, with $a=a_{0}$, are consequences of F1, F2 and F3; (see Sect. 4.2).

As for (1.10) and (1.11), the condition F4, (and the endpoint $\infty$ in F3), is typically more than we need in general and can be dispensed with in most of our estimates. When considering the Eq. (1.1), it will be enough to assume instead $F(t r)>B(\cdot, u, D u)$ for $r \in \Gamma$ and sufficiently large $t$, (depending on $r$ ), in accordance with [10].

In our scenario, we call $M[u]:=D^{2} u-A(\cdot, u, D u)$ the augmented Hessian matrix. Usually, we denote the elements of $M[u]$ and the matrix $F_{r}$ in F1 by $w_{i j}=D_{i j} u-A_{i j}$ and $F^{i j}$ respectively. A function $u$ is called admissible in $\Omega(\bar{\Omega})$ if

$$
M[u] \in \Gamma, \quad \text { in } \Omega,(\bar{\Omega}),
$$

so that the operator $\mathcal{F}$ satisfying F1 is elliptic with respect to $u$ in $\Omega(\bar{\Omega})$ when (1.12) holds. It is also clear that if $M[u] \in \bar{\Gamma}$ with $B \in F(\Gamma)$ in $\Omega(\bar{\Omega})$ then (1.1) is elliptic with respect to $u$ in $\Omega(\bar{\Omega})$, namely we require $B>a_{0}$ in $\Omega(\bar{\Omega})$ for $F$ satisfying F3.

An important ingredient for regularity of solutions to equations involving the augmented matrix $M[u]$ is the co-dimension one convexity (strict convexity) condition on the matrix $A$ with respect to $p$, that is

$$
A_{i j}^{k l}(x, z, p) \xi_{i} \xi_{j} \eta_{k} \eta_{l} \geq 0,(>0),
$$

for all $\xi, \eta \in \mathbb{R}^{n}, \xi \perp \eta$, where $A_{i j}^{k l}=D_{p_{k} p_{l}}^{2} A_{i j}$ and $A$ is twice differentiable at $(x, z, p) \in \Omega \times \mathbb{R} \times \mathbb{R}^{n}$. Note that we use the standard summation convention throughout this paper that repeated indices indicate summation from 1 to $n$ unless otherwise specified. As in $[17,18,40]$, we also call the matrix $A$ regular (strictly regular) at $x \in \bar{\Omega}$ if $A$ satisfies (1.13) for all $(z, p) \in \mathbb{R} \times \mathbb{R}^{n}$. These conditions were originally formulated for optimal transportation problems in the Monge-Ampère case, $k=n$, in $[31,45]$. The strictly regular condition may also be viewed as a supplementary ellipticity.

We now start to formulate the main theorems in this paper. First we state a local/global second derivative estimate which extends the Monge-Ampère case in [31] and whose global version is needed for our treatment of the boundary condition (1.2).

Theorem 1.1 Let $u \in C^{4}(\Omega)$ be an admissible solution of Eq. (1.1) in $\Omega$. Assume one of the following conditions:

(i) F1, F2, F3 and $\mathrm{F5}^{+}$hold;

(ii) F1, F2, F3, F5 hold, and $B$ is convex with respect to $p$.

Then for any domains $\Omega^{\prime} \subset \subset \Omega_{0}$ in $\mathbb{R}^{n}, A \in C^{2}\left(\bar{\Omega} \times \mathbb{R} \times \mathbb{R}^{n}\right)$ strictly regular in $\bar{\Omega} \cap \Omega_{0}, B>a_{0}, \in C^{2}\left(\bar{\Omega} \cap \Omega_{0} \times \mathbb{R} \times \mathbb{R}^{n}\right)$, we have the estimate

$$
\sup _{\Omega \cap \Omega^{\prime}}\left|D^{2} u\right| \leq \sup _{\partial \Omega \cap \Omega_{0}}\left|D^{2} u\right|+C,
$$


for $u \in C^{4}\left(\bar{\Omega} \cap \Omega_{0}\right)$, where the constant $C$ depends on $n, A, B, F, \Gamma, \Omega \cap \Omega_{0}, \Omega \cap \Omega^{\prime}$ and $|u|_{1 ; \Omega}$.

The estimate (1.14) in Theorem 1.1 in the case $\Omega^{\prime}=\Omega$ provides us a global estimate which reduces the bound for second derivatives to the boundary. When $\Omega_{0}=\Omega$ we get the usual form of the interior estimate, which is already formulated for case (i) in [40]. A more precise version involving cut-off functions will be presented in Sect. 2. For the boundary estimates we need to assume appropriate geometric assumptions on the domain $\Omega$. We consider the operator $\mathcal{F}$ in (1.1) and domains $\Omega \subset \mathbb{R}^{n}$ with $\partial \Omega \in C^{2}$ and $v$ denoting the unit inner normal to $\partial \Omega, \delta=D-(\nu \cdot D) v$ the tangential gradient in $\partial \Omega$, and $P=I-v \otimes v$ the projection matrix onto the tangent space on $\partial \Omega$, where $I$ is the $n \times n$ identity matrix. (Here the tangential gradient operator is the vector operator $\delta=\left(\delta_{1}, \ldots, \delta_{n}\right)$ with $\delta_{i}=\left(\delta_{i j}-v_{i} v_{j}\right) D_{j}$, where $\delta_{i j}$ is the usual Kronecker delta). Then, for $A \in C^{1}\left(\partial \Omega \times \mathbb{R} \times \mathbb{R}^{n}\right)$, we introduce the $A$-curvature matrix on $\partial \Omega$,

$$
K_{A}[\partial \Omega](x, z, p)=-\delta v(x)+P\left(D_{p} A(x, z, p) \cdot v(x)\right) P .
$$

We call $\partial \Omega$ uniformly ( $\Gamma, A, G$ )-convex with respect to an interval valued function $\mathcal{I}(x)$ on $\partial \Omega$, if

$$
K_{A}[\partial \Omega](x, z, p)+\mu \nu(x) \otimes v(x) \in \Gamma,
$$

for all $x \in \partial \Omega, z \in \mathcal{I}(x), G(x, z, p) \geq 0$ and some $\mu=\mu(x, z, p)>0$. For a given function $u_{0}$, if we take $\mathcal{I}=\left\{u_{0}\right\}$ in the above definition, then $\partial \Omega$ is called uniformly $(\Gamma, A, G)$-convex with respect to $u_{0}$. For the cases $\Gamma=\Gamma_{k}$, corresponding to the $k$-Hessians and their quotients, (1.16) is equivalent to $K_{A}[\partial \Omega](x, z, p) \in \Gamma_{k-1}$. Moreover in the Monge-Ampère case, $k=n$, we recover our definitions of uniform ( $A, G$ )-convexity in [16], (called "uniform $A$-convexity with respect to $G$ " there), which extend the notion of uniform $c$-convexity with respect to a target domain $\Omega^{*}$ in the optimal transportation case, as introduced in [45]. When the interval $\mathcal{I}=\mathbb{R}$, we will simply call $\partial \Omega$ uniformly $(\Gamma, A, G)$-convex. This includes the case when $A$ and $G$ are independent of $z$ as then the interval $\mathcal{I}$ becomes irrelevant.

As in [16], we will assume that the function $G \in C^{2}\left(\partial \Omega \times \mathbb{R} \times \mathbb{R}^{n}\right)$ is concave in $p$, that is $G_{p p} \leq 0$ in $\partial \Omega \times \mathbb{R} \times \mathbb{R}^{n}$. This includes the quasilinear case, when $G_{p p}=0$,

$$
G(x, z, p)=\beta(x, z) \cdot p-\varphi(x, z),
$$

where $\beta=G_{p}$ and $\varphi$ are defined on $\partial \Omega \times \mathbb{R}$. If $G_{p p}(\cdot, u, D u) \leq 0$ on $\partial \Omega$ for $u \in C^{1}(\bar{\Omega})$ then we say that $G$ is concave in $p$, with respect to $u$. Note that we define the obliqueness in (1.3) with respect to the unit inner normal $v$, so that our function $G$ keeps the same sign with those in [16] and is the negative of that in $[41,45,48]$. When $G$ is nonlinear in $p$ we will assume a further structural condition on $F$.

F6 $\mathcal{E}_{2}:=F_{r_{i j}} r_{i k} r_{j k} \leq o(|r|) \mathscr{T}$, uniformly for $a_{0}<a \leq F(r) \leq b$, as $|r| \rightarrow \infty$.

We remark that the Hessian operators $F_{k}(k=2, \ldots, n)$ and the Hessian quotients $F_{n, k}(1 \leq l \leq n-1)$ satisfy F6 in the positive cone $K^{+}[50]$. Further examples are 
given in Sects. 4.2 and 4.3. To complete our hypotheses, we will also assume for the second derivative bounds in this paper, (unless F6 is satisfied), that the cone $\Gamma$ lies strictly in a half space in the sense that $r \leq \operatorname{trace}(r) I$ for all $r \in \Gamma$, that is $\Gamma \subset \mathcal{P}_{n-1}$ in accordance with our examples in Sect. 4.2. This property is satisfied by the cones $\Gamma_{k}$ for $k \geq 2$, (but excludes the already well known quasilinear case when $k=1$ ). We now state the global second derivative bound which can be viewed as the main result of this paper.

Theorem 1.2 Let $u \in C^{4}(\Omega) \cap C^{3}(\bar{\Omega})$ be an admissible solution of the boundary value problem (1.1)-(1.2) in a $C^{3}$ domain $\Omega \subset \mathbb{R}^{n}$, with boundary $\partial \Omega$ uniformly $(\Gamma, A, G)$ convex with respect to $u$. Assume that $F$ satisfies conditions $F 1-F 5, A \in C^{2}(\bar{\Omega} \times \mathbb{R} \times$ $\left.\mathbb{R}^{n}\right)$ is strictly regular in $\bar{\Omega}, B>a_{0}, \in C^{2}\left(\bar{\Omega} \times \mathbb{R} \times \mathbb{R}^{n}\right), G \in C^{2}\left(\partial \Omega \times \mathbb{R} \times \mathbb{R}^{n}\right)$ is oblique and concave in $p$ with respect to u satisfying (1.3), and either (i) F5 $5^{+}$holds or (ii) $B$ is independent of $p$. Assume further that either $\mathcal{G}$ is quasilinear and $\Gamma \subset \mathcal{P}_{n-1}$ or F also satisfies F6. Then we have the estimate

$$
\sup _{\Omega}\left|D^{2} u\right| \leq C
$$

where $C$ is a constant depending on $F, A, B, G, \Omega, \beta_{0}$ and $|u|_{1 ; \Omega}$.

Remark 1.1 A stronger condition than regularity of the matrix function $A$ is necessary in the above hypotheses as it is known from the Monge-Ampère case that one cannot expect second derivative estimates for general oblique boundary value problems for $A \equiv 0$, which is a special case of regular $A$ but not strictly regular, see $[47,52]$. We also remark that the alternative condition that $B$ is independent of $p$ may be replaced by $D_{p} B$ sufficiently small, as well as $B$ convex with respect to $p$, and we will see from our treatment in Sect. 2 that such a condition is reasonable. Analogously, we may also replace the condition that $G$ is quasilinear by $D_{p}^{2} G$ sufficiently small.

Remark 1.2 It should be noted that the feasibility of our uniform convexity condition depends on an effective relationship between the boundary operator $\mathcal{G}$ and the curvature matrix $K_{A}[\partial \Omega]$ to ensure at least that the matrix

$$
P\left(D_{p} A(x, z, p) \cdot v(x)\right) P
$$

is uniformly bounded from below, for all $x \in \partial \Omega, z \in \mathcal{I}(x), G(x, z, p) \geq 0$. More generally we need to impose a condition on the gradient $D u$, namely that there exists a sufficiently small boundary neighbourhood $\Omega_{\rho}=\{x \in \Omega \mid d(x)<\rho\}$, where $d(x)=\operatorname{dist}(x, \partial \Omega)$ and $\rho$ is a small positive constant, such that

$$
-\delta v\left(x^{\prime}\right)+P\left(D_{p} A(x, u(x), D u(x)) \cdot v\left(x^{\prime}\right)\right) P+\mu_{0} v\left(x^{\prime}\right) \otimes v\left(x^{\prime}\right) \in \Gamma,
$$

for all $x \in \Omega_{\rho}$ and $x^{\prime} \in \partial \Omega$ satisfying $d\left(x, x^{\prime}\right)=d(x)$ and $G\left(x^{\prime}, u(x), D u(x)\right) \geq 0$, for a positive constant $\mu_{0}$. In particular we would then obtain a bound (1.18) when the curvatures of $\partial \Omega$ are sufficiently large. Furthermore since the regularity of $A$ on $\partial \Omega$ implies the curvature matrix $K_{A}[\partial \Omega](x, z, p)$ is non-decreasing in $p \cdot v$, in the 
Neumann case, (1.17) with $\beta=v$, we need only assume that (1.16) holds for $x \in \partial \Omega$, $z=u(x), p=D u(x)$ and constant $\mu=\mu_{0}$, that is $x=x^{\prime} \in \partial \Omega$ in (1.19).

Remark 1.3 We may assume more generally that the matrix function $A$ and scalar function $B$ are only defined and $C^{2}$ smooth on some open set $\mathcal{U} \subset \mathbb{R}^{n} \times \mathbb{R} \times \mathbb{R}^{n}$, with $A$ strictly regular and $B>a_{0}$ in $\mathcal{U}$. Then Theorems 1.1 and 1.2 will continue to hold provided the one jet $J_{1}=J_{1}[u](\Omega)=(\cdot, u, D u)(\Omega)$ is strictly contained in $\mathcal{U}$, with the constants $C$ in the estimates (1.14) and (1.18) depending additionally on $\operatorname{dist}\left(J_{1}, \partial \mathcal{U}\right)$. This remark is particularly pertinent to examples arising from optimal transportation or geometric optics where often the resultant Monge-Ampère type equations are subject to constraints on $J_{1}[u]$ and moreover such constraints may determine an appropriate constant $\mu_{0}$ in (1.19).

In order to apply Theorem 1.2, to the existence of smooth solutions to (1.1)(1.2), we need gradient and solution estimates. Our conditions for gradient estimates are motivated by the case when $F$ is linear and the corresponding quadratic structure conditions for gradient estimates for uniformly elliptic quasilinear equations, as originally introduced by Ladyzhenskaya and Ural'tseva [7,21]. First we need additional conditions on either $A$ or $F$ which facilitate an analogue of uniform ellipticity.

To formulate the condition on the matrix function $A$, we first express the strict regularity condition (1.13) in the equivalent form,

$$
A_{i j}^{k l} \xi_{i} \xi_{j} \eta_{k} \eta_{l} \geq \lambda|\xi|^{2}|\eta|^{2}-\bar{\lambda}(\xi \cdot \eta)^{2}
$$

for all $\xi, \eta \in \mathbb{R}^{n}$, where $\lambda$ and $\bar{\lambda}$ are positive functions in $C^{0}\left(\Omega \times \mathbb{R} \times \mathbb{R}^{n}\right)$. To derive (1.20) from (1.13), we set $\eta^{\prime}=\eta-(\xi \cdot \eta) \xi$, for $|\xi|=|\eta|=1$, and apply (1.13) to the orthogonal vectors $\xi$ and $\eta^{\prime}$. We then call $A$ uniformly regular in $\Omega$, if $A$ is strictly regular in $\Omega$ and for any $M>0$, there exist positive constants $\lambda_{0}$ and $\bar{\lambda}_{0}$ such that

$$
\lambda(x, z, p) \geq \lambda_{0}, \quad \bar{\lambda}(x, z, p) \leq \bar{\lambda}_{0},
$$

for all $x \in \Omega,|z| \leq M, p \in \mathbb{R}^{n}$. The alternative condition on $F$ extends that introduced in the orthogonally invariant case for gradient estimates for curvature equations in [2].

F7 For a given constant $a>a_{0}$, there exists constants $\delta_{0}, \delta_{1}>0$ such that $F_{r_{i j}} \xi_{i} \xi_{j} \geq$ $\delta_{0}+\delta_{1} \mathscr{T}$, if $a \leq F(r)$ and $\xi$ is a unit eigenvector of $r$ corresponding to a negative eigenvalue.

We remark that F7 implies F5, with $b=\infty$, and moreover the Hessian quotients $F_{k, l}$, for $0 \leq l<k \leq n$ satisfy F7 in the cone $\Gamma_{k}$ with constants $\delta_{0}, \delta_{1}>0$, depending only on $k, l$ and $n[2,37]$.

We formulate (almost) quadratic growth conditions on $A$ and $B$ as follows.

$$
\begin{aligned}
& D_{x} A, D_{x} B, D_{z} A, D_{z} B=O\left(|p|^{2}\right), \quad D_{z} A \geq o\left(|p|^{2}\right) I, D_{z} B \geq o\left(|p|^{2}\right), \\
& \quad D_{p} A, D_{p} B=O(|p|),
\end{aligned}
$$


as $|p| \rightarrow \infty$, uniformly for $x \in \Omega,|z| \leq M$ for any $M>0$. Note that in the analogous natural growth conditions in the uniformly elliptic theory, the " $O$ " lower bounds on $D_{z} A$ and $D_{z} B$ in (1.22) can be dispensed with as a continuity estimate is available $[7,25]$. Also these are automatically satisfied under standard uniqueness conditions, namely when $A$ and $B$ are non-decreasing in $z$, that is $D_{z} A \geq 0$ and $D_{z} B \geq 0$.

We now state a gradient estimate for oblique semilinear boundary conditions, that is when $\beta$ in (1.17) is independent of $z$ so that (1.2) may be written in the form

$$
\mathcal{G}[u]=G(\cdot, u, D u)=\beta \cdot D u-\varphi(\cdot, u)=0, \quad \text { on } \partial \Omega .
$$

Some variants and extensions, including weaker versions of conditions (1.22), local gradient estimates and extensions to nonlinear $G$ will also be considered in conjunction with our treatment in Sect. 3.

Theorem 1.3 Let $u \in C^{3}(\Omega) \cap C^{2}(\bar{\Omega})$ be an admissible solution of the boundary value problem (1.1)-(1.2) for an oblique, semilinear boundary operator $\mathcal{G}$ in a bounded $C^{3}$ domain $\Omega \subset \mathbb{R}^{n}$. Assume that $\mathcal{F}$ satisfies $F 1$ and $F 3, A, B \in C^{1}\left(\bar{\Omega} \times \mathbb{R} \times \mathbb{R}^{n}\right)$, satisfy (1.22), $b_{0}:=\inf _{\Omega} B(\cdot, u, D u)>a_{0}$ and $\beta \in C^{2}(\partial \Omega), \varphi \in C^{2}(\partial \Omega \times \mathbb{R})$. Assume also one of the following further conditions:

(i) $A$ is uniformly regular, $\mathcal{F}$ satisfies $F 2$ and $F 5$, with $b=\infty$, and $B-p \cdot D_{p} B \leq$ $o\left(|p|^{2}\right)$ in (1.22);

(ii) $\mathcal{F}$ is orthogonally invariant satisfying $F 7, A=o\left(|p|^{2}\right)$ in (1.22) and $\beta=v$.

Then we have the estimate

$$
\sup _{\Omega}|D u| \leq C
$$

where $C$ is a constant depending on $F, A, B, \Omega, b_{0}, \beta, \varphi$ and $|u|_{0 ; \Omega}$.

As we will show in Sect. 3 the concavity condition F2 in Theorem 1.3 may be removed when $F$ is positive homogeneous of degree one and more generally. Note that the condition on $B$ in case (i) is automatically satisfied when $B$ is convex in $p$. Also when $B$ is bounded, we do not need to take $b=\infty$ in F5, while if the constants $\delta_{0}$ and $\delta_{1}$ in conditions F5 and F7 are independent of $a$, the constant $C$ in the estimate (1.24) does not depend on $b_{0}$. Analogously to the situation with uniformly elliptic equations, we obtain gradient estimates in terms of moduli of continuity when the " $O$ " is weakened to " $O$ " in the hypotheses, (1.22) and case (ii), of Theorem 1.3. In particular we will also prove a Hölder estimate for admissible functions in the cones $\Gamma_{k}$ for $k>n / 2$, when $A \geq O\left(|p|^{2}\right) I$, which extends our gradient estimate in the case $k=n$ in [16], Lemma 4.1. Taking account of this, as well as Theorems 1.2 and 1.3, we have, as an example of our consequent existence results, the following existence theorem for the augmented $k$-Hessian and Hessian quotient equations. In its formulation we will assume the existence of subsolutions and supersolutions to provide the necessary solution estimates and an appropriate interval $\mathcal{I}$ in our boundary convexity conditions. For this purpose we will say that functions $\underline{u}$ and $\bar{u}$, in $C^{2}(\Omega) \cap$ $C^{1}(\bar{\Omega})$, are respectively subsolution and supersolution of the boundary value problem 
(1.1)-(1.2) if

$$
\mathcal{F}[\underline{u}] \geq B(\cdot, \underline{u}, D \underline{u}), \quad \mathcal{F}[\bar{u}] \leq B(\cdot, \bar{u}, D \bar{u}),
$$

at points in $\Omega$, where they are admissible, and

$$
\mathcal{G}[\underline{u}] \geq 0, \quad \mathcal{G}[\bar{u}] \leq 0, \quad \text { on } \partial \Omega .
$$

Theorem 1.4 Let $\mathcal{F}=\mathcal{F}_{k, l}$ for some $0 \leq l<k \leq n, \Omega$ a bounded $C^{3,1}$ domain in $\mathbb{R}^{n}, A \in C^{2}\left(\bar{\Omega} \times \mathbb{R} \times \mathbb{R}^{n}\right)$ strictly regular in $\bar{\Omega}, B>0, \in C^{2}\left(\bar{\Omega} \times \mathbb{R} \times \mathbb{R}^{n}\right), \mathcal{G}$ semilinear and oblique, with $\beta \in C^{2,1}(\partial \Omega), \varphi \in C^{2,1}(\partial \Omega \times \mathbb{R})$. Assume that $\underline{u}$ and $\bar{u}, \in C^{2}(\Omega) \cap C^{1}(\bar{\Omega})$ are respectively an admissible subsolution and a supersolution of (1.1)-(1.2) with $\partial \Omega$ uniformly $\left(\Gamma_{k}, A, G\right)$-convex with respect to the interval $\mathcal{I}=$ $[\underline{u}, \bar{u}]$. Assume also that $A, B$ and $\varphi$ are non-decreasing in $z$, with at least one of them strictly increasing, and that $A$ and $B$ satisfy the quadratic growth conditions (1.22) with $D_{p} B=0$ if $l>0$. Then if one of the following further conditions is satisfied:

(i) $A$ is uniformly regular and $B-p \cdot D_{p} B \leq o\left(|p|^{2}\right)$ in (1.22);

(ii) $\beta=v$ and either (a) $A=o\left(|p|^{2}\right.$ ) in (1.22) or (b) $k>n / 2$ and $\Omega$ is convex;

(iii) $k=n$ and $A \geq O\left(|p|^{2}\right) I$ in place of (1.22),

there exists a unique admissible solution $u \in C^{3, \alpha}(\bar{\Omega})$ of the boundary value problem (1.1)-(1.2), for any $\alpha<1$.

Under the monotonicity hypotheses of Theorem 1.4, functions of the form

$$
\bar{u}=c_{0}+c_{1} \psi
$$

will be supersolutions of (1.1)-(1.2), for sufficiently large non-negative constants $c_{0}$ and $c_{1}$, where $\psi \in C^{2}(\bar{\Omega})$ is a defining function for $\Omega$, satisfying $\psi<0$ in $\Omega, \psi=0$, $D \psi \neq 0$ on $\partial \Omega$, assuming also $c_{1}=0$ and $F\left[-A\left(\cdot, c_{0}, 0\right)\right] \leq B\left(\cdot, c_{0}, 0\right)$, whenever $-A\left(\cdot, c_{0}, 0\right) \in \Gamma$, in the case when only $\varphi$ is strictly increasing in $z$. Using (1.27), we can then replace the interval $[\underline{u}, \bar{u}]$ in the hypotheses of Theorem 1.4 by $\left[\underline{u}, c_{0}\right]$.

This paper is organised as follows. In Sect. 2, we first prove the local/global second derivative estimate, Theorem 1.1, as well as an extension to non-constant vector fields, Lemma 2.1. Then in Sect. 2.2, by delicate analysis of the second derivatives on the boundary, we complete the proof of Theorem 1.2 through Lemmas 2.2 and 2.3 which treat respectively the estimation of non-tangential and tangential second derivatives. In the proof of Lemma 2.3 the strict regularity condition is crucial. In Sect. 3, we first prove the global gradient estimate, Theorem 1.3, under various more general structural assumptions on $F, A$ and $B$. Following this, in Sect. 3.2, we prove the analogous local gradient estimates in Theorem 3.1. In Sect. 3.3 we derive a Hölder estimate for admissible functions in the cones $\Gamma_{k}$ for $k>n / 2$, from which we can infer gradient estimates under natural quadratic growth conditions. In Sect. 4 , we prove existence theorems, Theorems 4.1 and 4.2 for semilinear and nonlinear oblique boundary value problems based on the a priori derivative estimates, which include Theorem 1.4 as a special case. We then present in Sect. 4.2 various examples of operators $\mathcal{F}$, matrices 
$A$, and boundary operators $\mathcal{G}$ along with the application to conformal geometry, where we relax the umbilic boundary restriction for second derivative estimates in Yamabe problems with boundary as studied in $[3,19]$. Furthermore we show in Sect. 4.3 that our theory can be applied to degenerate elliptic equations, where $F$ is only assumed non-decreasing in F1, with resultant solutions $u \in C^{1,1}(\bar{\Omega})$; see Corollary 4.1, and provide a particular example in Corollaries 4.2 and 4.3. As indicated at the end of Section 4.3, these results also embrace degeneracies when $b=a_{0}$. In Sect. 4.4, we conclude this paper with some final remarks which also foreshadow further results.

We also point out here that in our formulations and proofs we have generally assumed for simplicity that the functions $A, B, G$ and domains $\Omega$ are $C^{k}$ smooth for appropriate $k$, although typically this can be replaced by $C^{k-1,1}$ smooth, as indicated in Remark 4.2. Since our main concern here is a priori estimates for classical solutions the reader may simply assume that all functions and domains are $C^{\infty}$ smooth.

\section{Second derivative estimates}

We introduce some notation and proceed to the second order derivative estimates for admissible solutions $u$ of (1.1)-(1.2). We denote the augmented Hessian $M[u]$ by $w=\left\{w_{i j}\right\}$, that is

$$
w_{i j}=D_{i j} u-A_{i j}(\cdot, u, D u)
$$

As usual we denote the first and second partial derivatives of $F$ at $M[u]$ by $F^{i j}$ and $F^{i j, k l}$, namely

$$
F^{i j}=\frac{\partial F}{\partial r_{i j}}(M[u]), \quad F^{i j, k l}=\frac{\partial^{2} F}{\partial r_{i j} \partial r_{k l}}(M[u]) .
$$

Then for an admissible $u$, we know from $\mathrm{F} 1$ that the matrix $\left\{F^{i j}\right\}$ is positive definite and from $\mathrm{F} 2$ that

$$
F^{i j, k l} \eta_{i j} \eta_{k l} \leq 0
$$

for all $\left\{\eta_{i j}\right\} \in \mathbb{S}^{n}$. Let us also denote $\mathscr{T}=\operatorname{trace}\left(F_{r}\right)=\sum_{i=1}^{n} F^{i i}$ so that by positivity $\left|F_{r}\right| \leq \mathscr{T}$.

It will also be convenient here to use (1.20) to express the strict regularity of $A$, with respect to $u$, in the form

$$
A_{i j}^{k l}(\cdot, u, D u) \xi_{i} \xi_{j} \eta_{k} \eta_{l} \geq c_{0}|\xi|^{2}|\eta|^{2}-c_{1}(\xi \cdot \eta)^{2},
$$

for arbitrary vectors $\xi, \eta \in \mathbb{R}^{n}$, where $c_{0}$ and $c_{1}$ are positive constants depending on $A$ and $\sup (|u|+|D u|)$. Then for any positive symmetric matrix $\left\{F^{i j}\right\}$ with eigenvalues $\lambda_{1}, \ldots, \lambda_{n}>0$ and corresponding eigenvectors, $\phi^{1}, \ldots, \phi^{n}$, we can write 


$$
\begin{aligned}
F^{i j} A_{i j}^{k l} \eta_{k} \eta_{l} & =\sum_{s=1}^{n} \lambda_{s} A_{i j}^{k l} \phi_{i}^{s} \phi_{j}^{s} \eta_{k} \eta_{l} \\
& \geq c_{0} \sum_{s=1}^{n} \lambda_{s}|\eta|^{2}-c_{1} \sum_{s=1}^{n} \lambda_{s}\left(\phi^{s} \cdot \eta\right)^{2} \\
& =c_{0} \mathscr{T}|\eta|^{2}-c_{1} F^{i j} \eta_{i} \eta_{j} .
\end{aligned}
$$

\subsection{Local/global second derivative estimates}

In this subsection, we derive the local and global second derivative estimates for admissible solutions of Eq. (1.1), and give the proof of Theorem 1.1. We will need to differentiate the Eq. (1.1), with respect to vector fields $\tau=\left(\tau^{1}, \ldots, \tau^{n}\right)$ with $\tau^{i} \in C^{2}(\bar{\Omega}), i=1, \ldots, n$. We introduce the linearized operators of the operator $\mathcal{F}$ and Eq. (1.1),

$$
L v:=F^{i j}\left[D_{i j} v-A_{i j}^{k} D_{k} v\right], \quad \mathcal{L} v:=L v-\left(D_{p_{k}} B(\cdot, u, D u)\right) D_{k} v
$$

for $v \in C^{2}(\Omega)$, where $A_{i j}^{k}=D_{p_{k}} A_{i j}(\cdot, u, D u)$. For convenience below we shall as usual denote partial derivatives of functions on $\Omega$ by subscripts, that is $u_{i}=D_{i} u, u_{\tau}=$ $D_{\tau} u=\tau^{i} u_{i}, u_{i j}=D_{i j} u, u_{i \tau}=u_{i j} \tau^{j}, u_{\tau \tau}=u_{i j} \tau^{i} \tau^{j}$, etc. Differentiating once we now obtain,

$$
\mathcal{L} u_{\tau}=F^{i j} \tilde{D}_{x_{\tau}} A_{i j}+\tilde{D}_{x_{\tau}} B+F^{i j}\left(2 \tau_{i}^{k} u_{j k}+\tau_{i j}^{k} u_{k}-A_{i j}^{k} \tau_{k}^{l} u_{l}\right)-\left(D_{p_{k}} B\right) \tau_{k}^{l} u_{l} .
$$

where $\tilde{D}_{x_{\tau}}=\tau \cdot \tilde{D}_{x}$ and $\tilde{D}_{x}=D_{x}+D u D_{z}$. Differentiating twice, we then obtain

$$
\begin{aligned}
\mathcal{L} u_{\tau \tau}= & -F^{i j, k l} D_{\tau} w_{i j} D_{\tau} w_{k l}+F^{i j}\left[\tau^{k} \tau^{l} \tilde{D}_{x_{k} x_{l}} A_{i j}+A_{i j}^{k l} u_{k \tau} u_{l \tau}+2\left(\tilde{D}_{x_{\tau}} A_{i j}^{k}\right) u_{k \tau}\right] \\
& +\left(D_{p_{k} p_{l}} B\right) u_{k \tau} u_{l \tau}+\tau^{k} \tau^{l} \tilde{D}_{x_{k} x_{l}} B+2\left(\tilde{D}_{x_{\tau}} D_{p_{k}} B\right) u_{k \tau} \\
& +F^{i j}\left[4 \tau_{i}^{k} u_{j k \tau}+\left(\tau^{k} \tau^{l}\right)_{i j} u_{k l}-2 A_{i j}^{s} \tau_{s}^{k} u_{k \tau}\right]-2\left(D_{p_{s}} B\right) \tau_{s}^{k} u_{k \tau}
\end{aligned}
$$

where $D_{p} \tilde{D}_{x}=\tilde{D}_{x} D_{p}$ is used. To derive the local and global estimates in Theorem 1.1, we only need $\tau$ to be a constant unit vector, in which case the last two terms in (2.6) and (2.7) are not present. Setting

$$
v_{1}=u_{\tau \tau}+\frac{1}{2} c_{1}\left|u_{\tau}\right|^{2}
$$

we then have from the concavity F2 and strict regularity (2.3),

$$
\mathcal{L} v_{1} \geq c_{0} \mathscr{T}\left|D u_{\tau}\right|^{2}-C(1+\mathscr{T})\left(1+\left|D u_{\tau}\right|\right)+\lambda_{B}\left|D u_{\tau}\right|^{2}
$$

where $C$ is a constant depending on $n,|A|_{C^{2}},|B|_{C^{2}}$ and $|u|_{1 ; \Omega}$ and $\lambda_{B}$ is the minimum eigenvalue of the matrix $D_{p}^{2} B$. Invoking conditions $\mathrm{F}^{+}$, or $\mathrm{F} 5$ and convexity of $B$ 
in $p$, we then have from the classical maximum principle, under the hypotheses of Theorem 1.1,

$$
\sup _{\Omega} u_{\tau \tau} \leq \sup _{\partial \Omega}\left|u_{\tau \tau}\right|+C
$$

which implies a global upper bound for $D^{2} u$, since $\tau$ can be any unit vector. To get the corresponding local estimate, we fix a function $\zeta \in C^{2}\left(\mathbb{R}^{n}\right)$, satisfying $0 \leq \zeta \leq 1$ and define,

$$
v=\zeta^{2} v_{1}=\zeta^{2}\left(u_{\tau \tau}+\frac{1}{2} c_{1}\left|u_{\tau}\right|^{2}\right)
$$

From the inequality (2.9) we obtain the corresponding inequality for $v$ at a maximum point, namely

$$
\mathcal{L} v \geq c_{0} \mathscr{T} \zeta^{2}\left|D u_{\tau}\right|^{2}-C(1+\mathscr{T})\left(1+\zeta^{2}\left|D u_{\tau}\right|+\left|u_{\tau \tau}\right|\right)+\lambda_{B} \zeta^{2}\left|D u_{\tau}\right|^{2}
$$

where $C$ depends additionally on $|\zeta|_{2 ; \Omega}$, so that extending (2.10), we have

$$
\sup _{\Omega}\left(\zeta^{2} u_{\tau \tau}\right) \leq \sup _{\partial \Omega}\left(\zeta^{2}\left|u_{\tau \tau}\right|\right)+C
$$

The lower bounds follow from the concavity F2 since for a fixed matrix $r_{0} \in \Gamma$, for example $r_{0}=I$, and positive matrix $a_{0}^{i j}=F_{r_{i j}}\left(r_{0}\right)$, we have

$$
\begin{aligned}
a_{0}^{i j} u_{i j} & =a_{0}^{i j}\left(w_{i j}+A_{i j}\right) \\
& \geq F(w)-F\left(r_{0}\right)+\sum_{i=1}^{n} a_{0}^{i i}+a_{0}^{i j} A_{i j} \geq-C
\end{aligned}
$$

by virtue of (1.1). Taking $\tau$ to be an eigenvector of $\left\{a_{0}^{i j}\right\}$, we infer the full bound from the upper bound (2.13). Hence we conclude the estimate

$$
\sup _{\Omega}\left(\zeta^{2}\left|D^{2} u\right|\right) \leq \sup _{\partial \Omega}\left(\zeta^{2}\left|D^{2} u\right|\right)+C
$$

Theorem 1.1 now follows by taking $\zeta \in C_{0}^{2}\left(\Omega_{0}\right)$ and $\zeta=1$ on $\Omega^{\prime}$.

The one-sided estimate (2.13) can be extended to non-constant vector fields $\tau$ when $\mathcal{F}$ is orthogonally invariant. Moreover the relevant calculations will be critical for us in the proof of tangential boundary estimates when $G$ is nonlinear in $p$.

Lemma 2.1 Assume in addition to the hypotheses of Theorem 1.1 that the operator $\mathcal{F}$ is orthogonally invariant. Then the estimate (2.13) holds for any vector field $\tau$ with skew symmetric Jacobian, with the constant $C$ depending additionally on $|\tau|_{1 ; \Omega \text {. }}$.

Proof First we note that the Jacobian $D \tau=\left\{\tau_{j}^{i}\right\}$ will in fact be a constant skew symmetric matrix so that $\tau$ itself is an affine mapping. Consequently the second derivatives 
of $\tau$ in (2.6) and (2.7) will vanish. Our main concern now is to control the third derivatives of $u$ in the last line of (2.7) and for this we adapt the key identities in the proof of Lemma 2.1 in [10], which follow by differentiating $F\left(P_{\alpha} r P_{\alpha}^{t}\right)$, for $r \in \Gamma$, with respect to $\alpha$ and setting $\alpha=0$, where $P_{\alpha}=\exp (\alpha D \tau)$ is orthogonal by virtue of the skew symmetry of $D \tau$. Thus taking $r_{i j}=w_{i j}$, we have

$$
F^{i j} \tau_{i}^{k} w_{j k}=0
$$

and

$$
F^{i j}\left(\tau_{i}^{k} \tau_{j}^{l} w_{k l}+\tau_{i}^{k} \tau_{k}^{l} w_{j l}\right)+2 F^{i j, k l} \tau_{i}^{s} w_{j s} \tau_{k}^{t} w_{l t}=0
$$

Differentiating (2.16) with respect to $\tau$, we have

$$
F^{i j} \tau_{i}^{k} D_{\tau} w_{j k}+F^{i j, k l} \tau_{i}^{s} w_{j s} D_{\tau} w_{k l}=0
$$

From (2.17) and (2.18), we then obtain

$$
\begin{aligned}
- & F^{i j, k l} D_{\tau} w_{i j} D_{\tau} w_{k l}+2 F^{i j}\left[2\left(\tau_{i}^{k}\right) D_{\tau} w_{j k}+\tau_{i}^{k} \tau_{j}^{l} w_{k l}\right] \\
& =-F^{i j, k l}\left(D_{\tau} w_{i j}+2 \tau_{i}^{s} w_{j s}\right)\left(D_{\tau} w_{k l}+2 \tau_{k}^{t} w_{l t}\right)-2 F^{i j} \tau_{i}^{k} \tau_{k}^{l} w_{j l} \\
& \geq-2 F^{i j} \tau_{i}^{k} \tau_{k}^{l} w_{j l}
\end{aligned}
$$

by the concavity F2. Substituting into (2.7), using the definition $w_{i j}=u_{i j}-A_{i j}$ and following our previous argument for constant $\tau$, we would obtain the upper bound (2.13), with constant $C$ replaced by $C\left(1+\sqrt{M}_{2}\right)$ where $M_{2}=\sup _{\Omega}\left|D^{2} u\right|$.

In order to get the full strength of Lemma 2.1, we need to control the last term in (2.19). Note that this term is nonnegative if $\Gamma=\Gamma_{n}$ or in the special case when $\left|D \tau^{i}\right|=\tau_{0}, i=1, \ldots, n$, for a constant $\tau_{0}$, since

$$
-F^{i j} \tau_{i}^{k} \tau_{k}^{l} w_{j l} \geq\left(\tau_{0}\right)^{2} F^{i j} w_{i j} \geq 0
$$

from (1.10). In general we proceed by calculating

$$
\mathcal{L}\left(\tau^{l} \tau_{l}^{k} u_{k}\right)=F^{i j}\left(2 \tau_{i}^{k} \tau_{k}^{l} u_{j l}-A_{i j}^{s} \tau_{s}^{k} \tau_{k}^{l} u_{l}\right)-\left(D_{p_{s}} B\right) \tau_{s}^{k} \tau_{k}^{l} u_{l}+\tau^{l} \tau_{l}^{k} \mathcal{L} u_{k} .
$$

Taking account of (2.6), (2.7), (2.19) and (2.20), we then obtain the differential inequality (2.12) with the function $u_{\tau \tau}$ in (2.11) replaced by the function

$$
\left(u_{\tau}\right)_{\tau}=u_{\tau \tau}+\tau^{l} \tau_{l}^{k} u_{k}
$$

from which Lemma 2.1 follows. Note that in the process of obtaining the inequality (2.12), there is a term $c_{1} \zeta^{2} u_{\tau} F^{i j} \tau_{i}^{k} w_{j k}$ which is identically equal to zero by using (2.16). 
We remark that for the Monge-Ampère operator, in the form $F(r)=\log (\operatorname{det} r)$, we can take $\tau$ to be any $C^{2}$ vector field in (2.13) with the constant $C$ now depending additionally on $|\tau|_{2 ; \Omega}$. This follows from the identity $F^{i k} r_{k j}=\delta_{i j}$.

\subsection{Boundary second derivative estimates}

To prove Theorem 1.2, we have to establish estimates for second derivatives on the boundary $\partial \Omega$ under the boundary condition (1.2). First we will consider the nontangential estimates and as in [16], the geometric convexity hypotheses on the domain $\Omega$ in Theorem 1.2 are crucial for this stage. We assume that the functions $G(\cdot, z, p)$ and $v$ have been extended to $\bar{\Omega}$, to be constant along normals to $\partial \Omega$ in some neighbourhood $\mathcal{N}$ of $\partial \Omega$. Differentiating the boundary condition (1.2) with respect to a tangential vector field $\tau$ we have

$$
\tilde{D}_{x_{\tau}} G+\left(D_{p_{k}} G\right) u_{k \tau}=0, \quad \text { on } \partial \Omega,
$$

and hence we have an estimate

$$
\left|u_{\tau \beta}\right| \leq C, \quad \text { on } \partial \Omega
$$

for any unit tangential vector field $\tau$, where $\beta=D_{p} G(\cdot, u, D u)$ and the constant $C$ depends on $G, \Omega$ and $|u|_{1 ; \Omega}$. The estimation of the pure second order oblique derivatives $u_{\beta \beta}$ is much more complicated. In general we can only obtain an estimate from above in terms of the tangential derivatives on the boundary. Setting

$$
M_{2}=\sup _{\Omega}\left|D^{2} u\right|, \quad M_{2}^{\prime}=\sup _{\partial \Omega|\tau|=1, \tau \cdot \nu=0} \sup _{\mid \tau \tau}\left|u_{\tau}\right|
$$

we formulate this as follows.

Lemma 2.2 Let $u \in C^{3}(\bar{\Omega})$ be an admissible solution of the boundary value problem (1.1)-(1.2) in a bounded $C^{2}$ domain $\Omega \subset \mathbb{R}^{n}$, with boundary $\partial \Omega$ uniformly $(\Gamma, A, G)$ convex with respect to $u$. Assume that $F$ satisfies conditions $F 1-F 5, A \in C^{1}(\bar{\Omega} \times \mathbb{R} \times$ $\left.\mathbb{R}^{n}\right), B>a_{0}, \in C^{1}\left(\bar{\Omega} \times \mathbb{R} \times \mathbb{R}^{n}\right), G \in C^{2}\left(\partial \Omega \times \mathbb{R} \times \mathbb{R}^{n}\right)$ is oblique with respect $u$ satisfying (1.3), either $\mathrm{F5}^{+}$holds or $B$ is independent of $p$ and either $\mathcal{G}$ is quasilinear satisfying (1.17) or F6 holds. Then for any $\epsilon>0$,

$$
\sup _{\partial \Omega} u_{\beta \beta} \leq \epsilon M_{2}+C_{\epsilon}\left(1+M_{2}^{\prime}\right)
$$

where $\beta=D_{p} G(\cdot, u, D u)$, and $C_{\epsilon}$ is a constant depending on $\epsilon, F, A, B, G, \Omega, \beta_{0}$ and $|u|_{1 ; \Omega}$. In the case when F6 holds, the estimate (2.23) holds without the dependence on $M_{2}^{\prime}$.

For any function $g \in C^{2}\left(\bar{\Omega} \times \mathbb{R} \times \mathbb{R}^{n}\right)$ and linearized operator $L$ in (2.5), by calculation we have 


$$
\begin{aligned}
L g(\cdot, u, D u)= & F^{i j}\left(D_{p_{k} p_{l}} g\right) u_{i k} u_{j l}+F^{i j}\left(D_{x_{i} x_{j}}-A_{i j}^{k} D_{x_{k}}\right) g \\
& +\left(D_{z} g\right) L u+\left(D_{p_{k}} g\right) L u_{k}+F^{i j}\left[2\left(\tilde{D}_{x_{i}} D_{p_{k}} g\right) u_{j k}\right. \\
& \left.+2\left(\tilde{D}_{x_{i}} D_{z} g\right) u_{j}-\left(D_{z z} g\right) u_{i} u_{j}\right],
\end{aligned}
$$

where

$$
L u=F^{i j} \delta_{i k} u_{j k}-F^{i j} A_{i j}^{k} u_{k},
$$

and

$$
L u_{k}=F^{i j} \tilde{D}_{x_{k}} A_{i j}+\tilde{D}_{x_{k}} B+\left(D_{p_{l}} B\right) u_{k l} .
$$

Plugging (2.25), (2.26) into (2.24), we obtain the differential inequality,

$$
L g \geq F^{i j}\left(D_{p_{k} p_{l}} g\right) u_{i k} u_{j l}-C \mathscr{T}+F^{i j} \tilde{\beta}_{i k} u_{j k}+\left(D_{p_{k}} g\right)\left(D_{p_{l}} B\right) u_{k l},
$$

in $\Omega$, with

$$
\tilde{\beta}_{i k}:=2 \tilde{D}_{x_{i}} D_{p_{k}} g+\left(D_{z} g\right) \delta_{i k}
$$

where the constant $C$ depends on $n, \Omega,|g|_{C^{2}},|A|_{C^{1}},|B|_{C^{1}}$ and $|u|_{1 ; \Omega}$. Note that when $a_{0}$ is finite, (2.25) can be estimated directly from (1.10).

Proof of Lemma 2.2. For any fixed boundary point $x_{0} \in \partial \Omega$, we consider the function

$$
\bar{v}=G(\cdot, u, D u)+\frac{a}{2}\left|D u-D u\left(x_{0}\right)\right|^{2},
$$

where $G$ is the boundary function in (1.2), and $a \leq 1$ is a positive constant. We consider the quasilinear case of $\mathcal{G},(1.17)$, namely $G_{p p}=0$. We also consider the case when $\mathrm{F}^{+}$holds. By (2.27), Cauchy's inequality and (2.26), we have

$$
\begin{aligned}
L \bar{v} \geq & -C \mathscr{T}+F^{i j} \tilde{\beta}_{i k} u_{j k}+\left(D_{p_{k}} G\right)\left(D_{p_{l}} B\right) u_{k l} \\
& +a F^{i j} u_{i k} u_{j k}+a\left(u_{k}-u_{k}\left(x_{0}\right)\right) L u_{k} \\
\geq & -C(1+a) \mathscr{T}-\frac{1}{a} F^{i j} \tilde{\beta}_{i k} \tilde{\beta}_{j k} \\
& +\left[D_{p_{k}} G+a\left(u_{k}-u_{k}\left(x_{0}\right)\right)\right]\left(D_{p_{l}} B\right) u_{k l} \\
\geq & -\left[\frac{C}{a}+\left(\epsilon_{1} M_{2}+C_{\epsilon_{1}}\right)\right] \mathscr{T}, \quad \text { in } \Omega,
\end{aligned}
$$

for any $\epsilon_{1}>0$, where $g$ is replaced by $G$ in $\tilde{\beta}_{i k}, \mathrm{~F}^{+}$is used in the last inequality, the constants $C$ depend on $n, \Omega,|G|_{C^{2}},|A|_{C^{1}},|B|_{C^{1}}$ and $|u|_{1 ; \Omega}$, and the constant $C_{\epsilon_{1}}$ depends on $\epsilon_{1}, F$ and $B$.

We shall construct a suitable upper barrier for $\bar{v}$ at the point $x_{0}$. We employ a function of the form 


$$
\bar{\phi}=\phi+\frac{b}{2}\left|x-x_{0}\right|^{2}, \quad \text { in } \Omega_{\rho}
$$

with

$$
\phi=c\left(d-t d^{2}\right)
$$

where $d=d(x)=\operatorname{dist}(x, \partial \Omega), \Omega_{\rho}=\{x \in \Omega \mid d(x)<\rho\}, b, c, t$ and $\rho$ are positive constants to be determined. Then, by calculation, we have

$$
\begin{aligned}
\frac{1}{2 t d-1} L \phi & =F^{i j}\left[c\left(-D_{i j} d+A_{i j}^{k} D_{k} d+\frac{2 t}{1-2 t d} D_{i} d D_{j} d\right)\right] \\
& \geq F^{i j}\left[c\left(-D_{i j} d+A_{i j}^{k} D_{k} d+2 t D_{i} d D_{j} d\right)\right], \quad \text { in } \Omega_{\rho},
\end{aligned}
$$

provided $t \rho \leq 1 / 4$. For convenience, we denote $h_{i j}=-D_{i j} d+A_{i j}^{k} D_{k} d+2 t D_{i} d D_{j} d$ in (2.32). By the uniform $(\Gamma, A, G)$-convexity of $\partial \Omega$, since $|u|$ and $|D u|$ are bounded in $\Omega$, there exists a small positive constant $\sigma$ such that

$$
K_{A}[\partial \Omega](x, u, D u)+\mu_{0} v(x) \otimes v(x)-2 \sigma I \in \Gamma,
$$

for all $x \in \partial \Omega$ satisfying $G(x, u(x), D u(x)) \geq 0$. Reversing the projection onto the tangent space of $\partial \Omega$, we then have

$$
-D v(x)+D_{p} A(x, u, D u) \cdot v(x)+\tilde{\mu}_{0} v(x) \otimes v(x)-2 \sigma I \in \Gamma,
$$

for all $x \in \partial \Omega$, for a larger constant $\tilde{\mu}_{0}$, which implies $\left(h_{i j}-\sigma \delta_{i j}\right) \in \Gamma$ for $x \in \partial \Omega$ provided $t \geq \tilde{\mu}_{0}$. By choosing $\rho$ sufficiently small and then $t$ sufficiently large, we have $\left(h_{i j}-\sigma \delta_{i j}\right) \in \Gamma$ in $\Omega_{\rho}^{+}=\Omega_{\rho} \cap\{G(\cdot, u, D u)>0\}$. Note that the constants $\rho$ and $t$ should be chosen under the restriction $t \rho \leq 1 / 4$. Then the constants $\rho$ and $t$ are now fixed. Then, by the concavity F2, we have from (2.32),

$$
\begin{aligned}
\frac{1}{2 t d-1} L \phi & \geq F^{i j}\left[c\left(h_{i j}\right)\right] \\
& =c \sigma \mathscr{T}+F^{i j}\left[c\left(h_{i j}-\sigma \delta_{i j}\right)\right] \\
& \geq c \sigma \mathscr{T}+F\left(c\left(h_{i j}-\sigma \delta_{i j}\right)\right)-F\left(w_{i j}\right)+F^{i j} w_{i j} \\
& \geq c \sigma \mathscr{T}+F\left(c\left(h_{i j}-\sigma \delta_{i j}\right)\right)-B(\cdot, u, D u), \quad \text { in } \Omega_{\rho}^{+},
\end{aligned}
$$

where (1.1) and (1.10) are used in the last inequality. By using F4 with sufficiently large $c$, we have from $(2.35)$

$$
L \phi \leq-\frac{1}{2} \operatorname{cog}, \quad \text { in } \Omega_{\rho}^{+},
$$

where $2 t d-1 \leq-1 / 2$ in $\Omega_{\rho}^{+}$is used. Thus, we obtain, from (2.29),

$$
L \bar{\phi} \leq\left(-\frac{1}{2} c \sigma+C b\right) \mathscr{T} \leq L \bar{v}, \quad \text { in } \Omega_{\rho}^{+},
$$

provided $c \geq 2\left[C\left(b+\frac{1}{a}\right)+\left(\epsilon_{1} M_{2}+C_{\epsilon_{1}}\right)\right] / \sigma$. 
Next, we examine $\bar{v}$ and $\bar{\phi}$ on the boundary of $\Omega_{\rho}$. For $x \in \partial \Omega$, we have

$$
\begin{aligned}
\left|D u(x)-D u\left(x_{0}\right)\right| & \leq C\left(\sup _{\partial \Omega|\tau|=1, \tau \cdot \nu=0} \sup _{\tau}\left|D_{\tau} D u\right|\right)\left|x-x_{0}\right| \\
& \leq C\left(1+M_{2}^{\prime}\right)\left|x-x_{0}\right|,
\end{aligned}
$$

where the mixed derivative estimate (2.22) and the strict obliqueness (1.3) are used in the second inequality, so the constant $C$ depends also on $\beta_{0}$. For $x \in \Omega_{\rho}$ and $x^{\prime}$ the closest point on $\partial \Omega$, we then obtain,

$$
\begin{aligned}
\left|D u(x)-D u\left(x_{0}\right)\right|^{2} & \leq 4(\sup |D u|) M_{2} d(x)+2\left|D u\left(x^{\prime}\right)-D u\left(x_{0}\right)\right|^{2} \\
& \leq C\left(1+\left(M_{2}^{\prime}\right)^{2}+M_{2}\right)\left(\left|x-x_{0}\right|^{2}+d\right),
\end{aligned}
$$

so that

$$
\bar{v} \leq \frac{1}{2} a C\left(1+\left(M_{2}^{\prime}\right)^{2}+M_{2}\right)\left(\left|x-x_{0}\right|^{2}+d\right) \leq \bar{\phi}, \quad \text { on } \bar{\Omega}_{\rho} \cap\{G(\cdot, u, D u)=0\},
$$

by choosing $b=a C\left(1+\left(M_{2}^{\prime}\right)^{2}+M_{2}\right)$ and $c \geq b$. On the inner boundary, by choosing $c \geq C / \rho$, we have

$$
\bar{v} \leq \bar{\phi}, \quad \text { on } \partial \Omega_{\rho} \cap \Omega
$$

Now from $(2.37),(2.40)$ and $(2.41)$, by the comparison principle, we have

$$
\bar{v} \leq \bar{\phi}, \quad \text { in } \Omega_{\rho}^{+} .
$$

Since $\bar{v}\left(x_{0}\right)=\bar{\phi}\left(x_{0}\right)=0$, we have

$$
D_{\beta} \bar{v}\left(x_{0}\right) \leq D_{\beta} \bar{\phi}\left(x_{0}\right)
$$

which implies

$$
u_{\beta \beta}\left(x_{0}\right) \leq \beta^{0} c+C
$$

where $\beta^{0}:=\sup _{\partial \Omega}\left(G_{p}(\cdot, u, D u) \cdot v\right) \geq \beta_{0}$. We can fix the constant $c$ so that

$$
\begin{aligned}
c & \leq \frac{2\left[C\left(b+\frac{1}{a}\right)+\left(\epsilon_{1} M_{2}+C_{\epsilon_{1}}\right)\right]}{\sigma}+b+\frac{C}{\rho} \\
& \leq C\left[\left(\epsilon_{1}+a\right) M_{2}+a\left(M_{2}^{\prime}\right)^{2}+\frac{1}{a}\right]+C_{\epsilon_{1}},
\end{aligned}
$$

where $C$ now depends on $F, A, B, G, \Omega, \beta_{0}$ and $|u|_{1 ; \Omega}$, and $C_{\epsilon_{1}}$ depends additionally on $\epsilon_{1}$. For any $\epsilon>0$, taking $a=\frac{1}{1+\epsilon_{1} M_{2}}$ and $\epsilon_{1}=\frac{\epsilon}{\beta^{0} C}$ for a further constant $C$ in (2.45), we then get 


$$
u_{\beta \beta}\left(x_{0}\right) \leq \epsilon M_{2}+C_{\epsilon}\left(1+M_{2}^{\prime}\right)
$$

from (2.44) and (2.45). Since $x_{0}$ is any boundary point, we can take the supremum of (2.46) over $\partial \Omega$ to arrive at the desired estimate (2.23). Therefore, we have proved Lemma 2.2 in the case when $\mathcal{G}$ is quasilinear and $\mathrm{F}^{+}$holds. While in the case $\mathcal{G}$ is quasilinear and only F5 holds with $B$ independent in $p$, the last term in the second line of (2.29) does not appear. So we still arrive at the same estimate (2.23) and Lemma 2.2 is thus proved in the quasilinear case.

Next, we turn to the case that $F$ satisfies F6. Here we may simply take $a=0$ in (2.28) and $b=0$ in (2.30) so that from (2.27), F6 and Cauchy's inequality

$$
\begin{aligned}
L \bar{v} & \geq F^{i j}\left(D_{p_{k} p_{l}} G\right) u_{i k} u_{j l}-C \mathscr{T}+\left(D_{p_{k}} G\right)\left(D_{p_{l}} B\right) u_{k l}+2 F^{i j} \tilde{\beta}_{i k} u_{j k} \\
& \geq-\left(\epsilon_{1} M_{2}+C_{\epsilon_{1}}\right) \mathscr{T}, \quad \text { in } \Omega,
\end{aligned}
$$

for any $\epsilon_{1}>0$, where $\epsilon_{1}$ now comes from the use of both $\mathrm{F}^{+}$and F6, the constant $C$ depends on $n, \Omega,|G|_{C^{2}},|A|_{C^{1}},|B|_{C^{1}}$ and $|u|_{1 ; \Omega}$, and the constant $C_{\epsilon_{1}}$ depends also on $\epsilon_{1}$ and $F$. We can then derive the desired estimate (2.23), without the dependence on $M_{2}^{\prime}$, for both $\mathrm{F}^{+}$and $B$ independent of $p$.

Remark 2.1 The proof of Lemma 2.2 readily gives us a local boundary estimate when we only assume $\partial \Omega \cap B$ is uniformly ( $\Gamma, A, G$ )-convex with respect to $u$ for some ball $B=B_{R}\left(x_{0}\right)$ of radius $R$, centred at $x_{0}$. Under the hypotheses of Lemma 2.2, with $\Omega$ replaced by $\Omega \cap B$ and $\partial \Omega$ replaced by $\partial \Omega \cap B$, we then obtain, in place of (2.23),

$$
u_{\beta \beta}\left(x_{0}\right) \leq \epsilon M_{2 ; \Omega \cap B}+C_{\epsilon}\left(1+R^{-2}+M_{2 ; \partial \Omega \cap B}^{\prime}\right),
$$

where now $C_{\epsilon}$ is a constant depending on $\epsilon, F, A, B, G, \Omega, \beta_{0}$ and $|u|_{1 ; \Omega \cap B}$, and

$$
M_{2 ; \Omega \cap B}=\sup _{\Omega \cap B}\left|D^{2} u\right|, \quad M_{2 ; \partial \Omega \cap B}^{\prime}=\sup _{\partial \Omega \cap B|\tau|=1, \tau \cdot \nu=0} \sup _{\tau \tau}\left|u_{\tau \tau}\right|
$$

It now remains to estimate the pure tangential derivatives on the boundary. In this part, the strictly regular condition on the matrix $A$ is crucial. We can formulate the pure tangential derivative estimates as follows.

Lemma 2.3 Let $u \in C^{2}(\bar{\Omega}) \cap C^{4}(\Omega)$ be an admissible solution of the boundary value problem (1.1)-(1.2) in a $C^{3}$ domain $\Omega \subset \mathbb{R}^{n}$. Assume that $F, A$ and $B$ satisfy the hypothesis of Theorem 1.1 and $G \in C^{2}\left(\partial \Omega \times \mathbb{R} \times \mathbb{R}^{n}\right)$, with $G$ oblique and concave in $p$ with respect to $u$ satisfying (1.3), and either $\mathcal{G}$ is quasilinear or $\mathcal{F}$ is orthogonally invariant or $F$ also satisfies condition F6. Then for any tangential vector field $\tau$, $|\tau| \leq 1$ and constant $\epsilon>0$, we have the estimate

$$
M_{2}^{+}(\tau) \leq \epsilon M_{2}+C_{\epsilon},
$$

where $M_{2}^{+}(\tau)=\sup _{\partial \Omega} u_{\tau \tau}$, and $C_{\epsilon}$ is a constant depending on $\epsilon, F, A, B, G, \Omega, \beta_{0}$ and $|u|_{1 ; \Omega}$. 
Proof As usual we extend $v$ and $G$ smoothly to all of $\bar{\Omega}$ so that $v$ and $G(\cdot, z, p)$ are constant along normals to $\partial \Omega$ in some neighbourhood of $\partial \Omega$. Suppose that the function

$$
v_{\tau}=u_{\tau \tau}+\frac{c_{1}}{2}\left|u_{\tau}\right|^{2}
$$

takes a maximum over $\partial \Omega$ and tangential vectors $\tau$, such that $|\tau| \leq 1$, at a point $x_{0} \in \partial \Omega$ and vector $\tau=\tau_{0}$, where $c_{1}$ is the constant in the strict regularity condition (2.3). Without loss of generality, we may assume $x_{0}=0$ and $\tau_{0}=e_{1}$. Setting

$$
b=\frac{v_{1}}{\beta \cdot v}, \quad \tau=e_{1}-b \beta,
$$

we then have, at any point in $\partial \Omega$,

$$
v_{1}=v_{\tau}+b\left(2 u_{\beta \tau}+c_{1} u_{\beta} u_{\tau}\right)+b^{2}\left(u_{\beta \beta}+\frac{c_{1}}{2} u_{\beta}^{2}\right)
$$

with $v_{1}(0)=v_{\tau}(0), b(0)=0$ and $\tau(0)=e_{1}$. From (2.21), $u_{\beta \tau}=-\tilde{D}_{x_{\tau}} G$ on $\partial \Omega$ so that setting

$$
g=\frac{1}{\beta \cdot v}\left(2 u_{\beta \tau}+c_{1} u_{\beta} u_{\tau}\right),
$$

we have

$$
|g-g(0)| \leq C\left(1+M_{2}\right)|x|, \quad \text { on } \partial \Omega,
$$

where $C$ is a constant depending on $G, \Omega, \beta_{0}$ and $|u|_{1 ; \Omega}$. Accordingly, there exists a further constant $C_{1}$ depending on the same quantities, such that the function,

$$
\tilde{v}_{1}=v_{1}-g(0) v_{1}-C_{1}\left(1+M_{2}\right)|x|^{2}
$$

satisfies

$$
\begin{aligned}
\tilde{v}_{1} & \leq|\tau|^{2} \tilde{v}_{1}(0) \\
& \leq f \tilde{v}_{1}(0), \quad \text { on } \partial \Omega
\end{aligned}
$$

where $f$ is any non-negative function in $C^{2}(\bar{\Omega})$ satisfying $f \geq|\tau|^{2}$ on $\partial \Omega, f(0)=1$. In the case when $\mathcal{G}$ is quasilinear, that is $\beta=\beta(\cdot, u)$, we may simply estimate

$$
\begin{aligned}
|\tau|^{2} & \leq 1-2 b \beta_{1}+b^{2} \sup |\beta|^{2} \\
& \leq 1-2 \frac{\beta_{1}}{\beta \cdot v}(0) v_{1}+C_{1}|x|^{2}:=f .
\end{aligned}
$$

Now differentiating (1.2) twice in a tangential direction $\tau$, with $\tau(0)=e_{1}$, we obtain using the concavity of $G$ and (2.22), 


$$
D_{\beta} u_{11}(0) \geq-C_{1}\left(1+M_{2}\right) .
$$

Consequently for a sufficiently large constant $K$ depending on the same quantities as $C_{1}$, the function

$$
v=\tilde{v}_{1}-f \tilde{v}_{1}(0)-K\left(1+M_{2}\right) \phi
$$

must take an interior maximum in $\Omega$, where $\phi \in C^{2}(\bar{\Omega})$ is a negative defining function for $\Omega$ satisfying $\phi=0$ on $\partial \Omega, D_{\nu} \phi=-1$ on $\partial \Omega$. This effectively reduces our argument to the proof of Theorem 1.1. In the proof of Theorem 1.1, by replacing the function $v_{1}$ in (2.8) with the function $v$ in (2.57), we obtain at a maximum point $x_{0}$, using (2.3),

$$
u_{11}\left(x_{0}\right) \leq C \sqrt{1+M_{2}},
$$

where $C$ depends additionally on $A$ and $K|\phi|_{2 ; \Omega}$. The estimate (2.49) then follows by fixing $\phi$ and the constant $C_{1}$ in (2.55) so that $\phi \geq-\frac{\epsilon}{4 K}$ and $f \geq \frac{1}{2}$ in $\Omega$. Instead of adjusting $\phi$ we can alternatively maximize $v$ in a sufficiently small strip $\Omega_{\delta_{0}}$ around $\partial \Omega$ and apply the interior estimate in Theorem 1.1.

When $G$ is nonlinear in $p$, the coefficient $\beta_{1}$ in the expansion (2.55) of $|\tau|^{2}$ depends on $D u$ and cannot be controlled by the argument above. For orthogonally invariant $\mathcal{F}$, this is overcome by using a first order approximation to the tangent vector $e_{1}$ at 0 . Fixing the $x_{n}$ coordinate in the direction of $v$ at 0 , we then replace $e_{1}$ by the vector field

$$
\xi=e_{1}+\sum_{1 \leq k<n} \delta_{k} v_{1}(0)\left(x_{n} e_{k}-x_{k} e_{n}\right) .
$$

Then in place of (2.51), we have

$$
b=\frac{\xi \cdot v}{\beta \cdot v}, \quad \tau=\xi-b \beta,
$$

so that, both $b(0)=0, \delta b(0)=0$. Accordingly we then have, in place of (2.53) and (2.54),

$$
\tilde{v}_{1}:=v_{\xi}-C_{1}\left(1+M_{2}\right)|x|^{2} \leq \tilde{v}_{1}(0)\left(1+C_{1}|x|^{2}\right), \quad \text { on } \partial \Omega,
$$

where $C_{1}$ again denotes constants depending on $G, \Omega, \beta_{0}$ and $|u|_{1 ; \Omega}$. Comparing the forms of $\tilde{v}_{1}$ in (2.53) and (2.60), since $b(0)=0$ and $\delta b(0)=0$, here we can avoid the term $g(0) v_{1}$ in (2.60). The inequality in (2.60) is obtained by estimating $|\tau|^{2}=|\xi|^{2}-2 b(\xi \cdot \beta)+b^{2}|\beta|^{2}$, with $|\xi|^{2} \leq 1+2 \delta_{1} \nu_{1}(0) x_{n}+\sum_{1 \leq k<n}\left(\delta_{k} \nu_{1}(0)\right)^{2}|x|^{2}$ on $\partial \Omega,-2 b(\xi \cdot \beta)+b^{2}|\beta|^{2} \leq C_{1}|x|^{2}$ on $\partial \Omega$, (since $b(0)=0$ and $\delta b(0)=0$ ), and using $v(0)=e_{n}$ to estimate $x_{n}$ on $\partial \Omega$. Since the vector field $\xi$ has skew symmetric Jacobian $D \xi$, we can then reduce to the argument of the proof of Lemma 2.1 when $\mathcal{F}$ is orthogonally invariant. The reduction is achieved by replacing the function $v$ in 
(2.11), (where $u_{\tau \tau}$ is replaced by $\left(u_{\tau}\right)_{\tau}$ in the proof of Lemma 2.1), with the function $v$ in (2.57), (where $\tilde{v}_{1}$ is defined in (2.60), and $f$ is any non-negative function in $C^{2}(\bar{\Omega})$ satisfying $f \geq 1+C_{1}|x|^{2}$ on $\partial \Omega$ ). Combining (2.19) with (2.6) and (2.7), we would then, following our argument above, arrive at the estimate (2.58) at an interior maximum point $x_{0}$, with $e_{1}$ replaced by $\xi$ and from there get the corresponding estimate for $u_{\xi \xi}(0)=u_{11}(0)$ and conclude (2.49), as before.

Finally if F6 is satisfied, we first obtain from the formulae (2.24), (2.25) and (2.26), the complementary inequality to (2.27)

$$
\begin{aligned}
\mathcal{L} g & \leq C\left(\mathcal{E}_{2}+\mathscr{T}+1\right) \\
& \leq C\left(\epsilon M_{2}+1\right)(1+\mathscr{T}),
\end{aligned}
$$

for any $\epsilon>0$ and provided $\left|D^{2} u\right| \geq C_{\epsilon}$, where the term $F^{i j} \tilde{\beta}_{i k} u_{j k}$ in $\mathcal{L} g$ is treated in the same way as in (2.29). Here the constant $C$ depends on $\Omega,|g|_{C^{2}},|A|_{C^{1}},|B|_{C^{1}}$ and $|u|_{1 ; \Omega}$ while $C_{\epsilon}$ depends on $\epsilon, F$ and $|u|_{1 ; \Omega}$. Now taking $f=f(\cdot, u, D u)$ satisfying $f \geq|\tau|^{2}$ on $\partial \Omega, f \geq \frac{1}{2}, \in C^{2}\left(\bar{\Omega} \times \mathbb{R} \times \mathbb{R}^{n}\right)$, we then obtain at the interior maximum point $x_{0}$ of the function $v$ in (2.57), corresponding to (2.9),

$$
\mathcal{L} v \geq c_{0} \mathscr{T}\left|D u_{1}\right|^{2}-C(1+\mathscr{T})\left(1+\left|D u_{1}\right|+\epsilon M_{2}^{2}\right)+\lambda_{B}\left|D u_{1}\right|^{2},
$$

if $\left|u_{11}\left(x_{0}\right)\right| \geq C_{\epsilon}$. A suitable function $f$ for example is obtained by taking $f=$ $|\tau|^{2}+C_{1}|x|^{2}$ in $\Omega$ with $\beta \cdot v$ in (2.51) replaced by $\zeta(\beta \cdot v)$ where $\zeta \in C^{2}(\mathbb{R})$ satisfies $\zeta^{\prime}, \zeta^{\prime \prime} \geq 0, \zeta(t)=t$ for $t \geq 3 \beta_{0} / 4, \zeta(t)=\beta_{0} / 2$ for $t \leq \beta_{0} / 4$ and $C_{1}$ is a large enough constant, depending on $G, \Omega, \beta_{0}$ and $|u|_{1 ; \Omega}$, to ensure $f \geq \frac{1}{2}$. Also using F6 in conjunction with (2.3), we only need take $c_{1}=0$ in (2.50). By suitably adjusting $\epsilon$, we then infer the estimate (2.49).

With the local/global second derivative estimate in Theorem 1.1, and the boundary estimates in (2.22), Lemmas 2.2 and 2.3, we are now ready to prove the global second derivative estimate (1.18) in Theorem 1.2.

Proof of Theorem 1.2 In the quasilinear case we have by hypothesis that the sum of any $n-1$ eigenvalues of $w=M[u]$ is nonnegative which implies that the quantities $M_{2}^{\prime}$ and $M_{2}^{+}$are equivalent. Combining the estimates (2.22), (2.23) and (2.49), we then get an estimate

$$
\sup _{\partial \Omega} u_{\xi \xi} \leq \epsilon M_{2}+C_{\epsilon},
$$

for any constant unit vector $\xi$ and constant $\epsilon>0$ where $C_{\epsilon}$ depends on $\epsilon, F, A, B, G, \Omega, \beta_{0}$ and $|u|_{1 ; \Omega}$. Then using the concavity of $F$, as in the proof of Theorem 1.1, or the above property of $w$, we get the full boundary estimate

$$
\sup _{\partial \Omega}\left|D^{2} u\right| \leq \epsilon M_{2}+C_{\epsilon}
$$

and the desired estimate (1.18) follows from the global second derivative bound in Theorem 1.1 with $\epsilon$ chosen sufficiently small. If F6 is satisfied, the term in $M_{2}^{\prime}$ does 
not occur in the estimate (2.23) and we obtain (2.64) directly from the concavity of $F$, as in the proof of Theorem 1.1. This completes the proof of Theorem 1.2.

Remark 2.2 Using the equivalence of $M_{2}^{\prime}$ and $M_{2}^{+}$as above, we may replace $M_{2}$ by $u_{11}(0)$ in (2.56) and (2.57). Taking $\epsilon=1$ in our adjustment of $\phi$ in the proof of Lemma 2.3, we then obtain, in the cases when $\mathcal{G}$ is quasilinear or $\mathcal{F}$ is orthogonally invariant, a more precise version of the tangential estimate (2.49)

$$
M_{2}^{+}(\tau) \leq C\left(1+\sqrt{M_{2}}\right),
$$

where $C$ is a constant depending on $F, A, B, G, \Omega, \beta_{0}$ and $|u|_{1 ; \Omega}$.

\section{Gradient estimates}

In this section, we prove various gradient estimates for admissible solutions $u$ of the oblique problem (1.1)-(1.2). We mainly consider the case when the oblique boundary operator $\mathcal{G}$ is semilinear and in particular give the proof of Theorem 1.3. We also derive corresponding local gradient estimates as well as an estimate for nonlinear $\mathcal{G}$. As mentioned in the introduction, our conditions on either the matrix $A$ or the operator $F$ enable an analogue of uniform ellipticity. Accordingly we will employ improvements of the methods for uniformly elliptic equations in [25] with a critical adjustment used to supplement the tangential gradient terms in [25], which is similar to that used for gradient estimates in the conformal geometry case in [19]. We also prove a Hölder estimate for $\Gamma=\Gamma_{k}$ for $k>n / 2$, from which we infer gradient estimates under natural quadratic growth conditions.

\subsection{Global gradient estimates}

We consider the case of oblique semilinear $\mathcal{G}$ in (1.23) and normalise $G$ by dividing by $\beta \cdot v \geq \beta_{0}$, so that we can write

$$
G(x, z, p)=v \cdot p+\beta^{\prime} \cdot p^{\prime}-\varphi(x, z)
$$

where now $\beta^{\prime} \in C^{2}(\partial \Omega), \varphi \in C^{2}(\partial \Omega \times \mathbb{R})$ and $p^{\prime}=p-(p \cdot v) v$. For convenience, we still use $\varphi$ to denote its normalised form here. The boundary condition (1.2) can thus be written in the form

$$
\mathcal{G}[u]=G(\cdot, u, D u)=D_{\nu} u+\beta^{\prime} \cdot \delta u-\varphi(\cdot, u)=0, \quad \text { on } \partial \Omega .
$$

We begin with a preliminary calculation to estimate $\mathcal{L} g$ from below, for $g$ given by

$$
g(x, u, D u)=a_{k l}(x) u_{k} u_{l}+b_{k}(x, u) u_{k}+c(x, u),
$$

where $\left\{a_{k l}\right\}$ is a nonnegative matrix function on $\Omega, b(x, u)=\left(b_{1}(x, u), \ldots, b_{n}(x, u)\right)$ is a vector valued function on $\Omega \times \mathbb{R}, c(x, u)$ is a scalar function on $\Omega \times \mathbb{R}, a_{k l} \in C^{2}(\bar{\Omega})$, 
$b_{k}, c \in C^{2}(\bar{\Omega} \times \mathbb{R})$, and $u \in C^{3}(\Omega)$ is an admissible solution of (1.1). For this estimation we may assume more general growth conditions than (1.22), namely

$$
\begin{aligned}
& D_{x} A, D_{x} B, D_{z} A, D_{z} B=o\left(|p|^{3}\right), \quad D_{z} A \geq o\left(|p|^{2}\right) I, D_{z} B \geq o\left(|p|^{2}\right), \\
& \quad D_{p} A, D_{p} B=o\left(|p|^{2}\right)
\end{aligned}
$$

as $|p| \rightarrow \infty$, uniformly for $x \in \Omega,|z| \leq M$ for any $M>0$.

Now we calculate, using (2.24),

$$
\begin{aligned}
\mathcal{L} g= & 2 F^{i j} a_{k l} u_{i k} u_{j l}+u_{k} u_{l} \mathcal{L} a_{k l}+u_{l}\left[F^{i j} D_{x_{i} x_{j}} b_{l}-\left(F^{i j} A_{i j}^{k}+D_{p_{k}} B\right) D_{x_{k}} b_{l}\right] \\
& +\left[F^{i j} D_{x_{i} x_{j}} c-\left(F^{i j} A_{i j}^{k}+D_{p_{k}} B\right) D_{x_{k}} c\right]+\left(u_{k} D_{z} b_{k}+D_{z} c\right) \mathcal{L} u \\
& +\left[\left(2 a_{k l} u_{l}+b_{k}\right) \mathcal{L} u_{k}+2 F^{i j}\left(2 u_{l} D_{i} a_{k l}+\tilde{D}_{x_{i}} b_{k}\right) u_{j k}\right] \\
& +F^{i j}\left[2\left(u_{k} \tilde{D}_{x_{i}} D_{z} b_{k}+\tilde{D}_{x_{i}} D_{z} c\right) u_{j}-\left(u_{k} D_{z z} b_{k}+D_{z z} c\right) u_{i} u_{j}\right]
\end{aligned}
$$

where $\tilde{D}_{x_{i}}=D_{x_{i}}+u_{i} D_{z}$ and, corresponding to (2.25) and (2.26),

$$
\mathcal{L} u=F^{i j} \delta_{i k} u_{j k}-\left(F^{i j} A_{i j}^{k}+D_{p_{k}} B\right) u_{k}
$$

and

$$
\mathcal{L} u_{k}=F^{i j} \tilde{D}_{x_{k}} A_{i j}+\tilde{D}_{x_{k}} B .
$$

From the growth conditions (3.4), we then estimate

$$
\mathcal{L} g \geq 2 F^{i j} a_{k l} u_{i k} u_{j l}+F^{i j} \tilde{\beta}_{i k} u_{j k}-C(\mathscr{T}+1)\left(\omega(|D u|)|D u|^{4}+1\right),
$$

where

$$
\tilde{\beta}_{i k}=2\left(2 u_{l} D_{i} a_{k l}+\tilde{D}_{x_{i}} b_{k}\right)+\left(u_{l} D_{z} b_{l}+D_{z} c\right) \delta_{i k}
$$

and $\omega$ is a positive decreasing function on $[0, \infty)$ tending to 0 at infinity, depending on $A, B$ and $M_{0}=\sup _{\Omega}|u|$, and $C$ is a constant depending on $a_{k l}, b_{k}, c, \Omega, A, B$ and $M_{0}$. For our approach here we will assume $\left\{a_{k l}\right\}$ is positive definite so that $\left\{a_{k l}\right\} \geq a_{1} I$ for some positive constant $a_{1}$. By Cauchy's inequality and the positivity of $\left\{a_{k l}\right\}$, we then obtain

$$
\mathcal{L} g \geq a_{1} \mathcal{E}_{2}^{\prime}-C(\mathscr{T}+1)\left(\omega|D u|^{4}+1\right)
$$

where $\mathcal{E}_{2}^{\prime}=F^{i j} u_{i k} u_{j k}$.

In our proof of Theorem 1.3, we will specifically choose

$$
g=|\delta u|^{2}+|\mathcal{G}[u]|^{2}
$$

where $\nu$ and $G$ given by (3.1) are appropriately extended to all of $\bar{\Omega}$. For our purposes a convenient extension will be as usual to take $v$ constant along normals to $\partial \Omega$ in a 
sufficiently small strip $\{d<\rho\}$ where the distance function $d$ is $C^{2}$ smooth and to extend $\beta^{\prime} \in C^{2}(\bar{\Omega})$ and $\varphi \in C^{2}(\bar{\Omega} \times \mathbb{R})$ to vanish for $d \geq \rho$ so that $g=|D u|^{2}$ for $d \geq \rho$. Using Cauchy's inequality, we can estimate here

$$
\left\{a_{k l}\right\} \geq\left(1+\beta_{0}^{\prime}\right)^{-2} I
$$

where $\beta_{0}^{\prime}=\sup \left|\beta^{\prime}\right|$, so that (3.9) holds with $a_{1}=\left(1+\beta_{0}^{\prime}\right)^{-2}$ and $C$ depending on $\beta^{\prime}, \varphi, \Omega, A, B$ and $M_{0}$, with $\Omega \in C^{3}$. By further use of Cauchy's inequality, we also obtain

$$
\frac{a_{1}}{4}|D u|^{2}-|\varphi|^{2} \leq g \leq \frac{2}{a_{1}}|D u|^{2}+2|\varphi|^{2}
$$

so that the estimation of $D u$ is equivalent to that of the function $g$.

With these preparations, we give the proofs of the global gradient estimates.

Proof of Theorem 1.3. We employ auxiliary functions of the form

$$
v:=g+M_{1}^{2}(\alpha \eta-\kappa \phi),
$$

in $\Omega$, where $g$ is given by (3.10), $\eta=e^{+(-) K\left(u_{0}-u\right)}$, for a constant $u_{0}$ and a positive constant $K$ to be determined, $\phi \in C^{2}(\bar{\Omega})$ is a negative defining function for $\Omega$ satisfying $\phi=0$ on $\partial \Omega$ and $D_{\nu} \phi=-1$ on $\partial \Omega, M_{1}=\sup _{\Omega}|D u|, \alpha$ and $\kappa$ are positive constants to be determined. We assume the function $v$ attains its maximum over $\bar{\Omega}$ at some point $x_{0}$. If $x_{0} \in \partial \Omega$, then we have $D_{\beta} v\left(x_{0}\right) \leq 0$, where $\beta=v+\beta^{\prime}$. From the construction of $g$, we have on $\partial \Omega$,

$$
g=|\delta u|^{2}, \quad \text { and } D g=D\left(|\delta u|^{2}\right) .
$$

Then we have on $\partial \Omega$,

$$
\begin{aligned}
D_{\beta} v & =D_{\beta}|\delta u|^{2}+M_{1}^{2}\left(\alpha D_{\beta} \eta-\kappa D_{\beta} \phi\right) \\
& \geq 2 \delta_{k} u D_{\beta} \delta_{k} u+\kappa M_{1}^{2}-\alpha K \eta|\varphi|(\cdot, u) M_{1}^{2},
\end{aligned}
$$

using (3.14) and the boundary condition (3.2). Next by tangential differentiation of (3.2), as in (2.21), we have

$$
D_{\beta} \delta_{k} u=\delta_{x_{k}} \varphi+\left(D_{z} \varphi\right) \delta_{k} u-\left(\delta_{k} \beta_{i}\right) D_{i} u-\beta_{i} v_{k}\left(D_{i} v_{l}\right) D_{l} u-\beta_{i}\left(D_{i} v_{k}\right) D_{\nu} u .
$$

Plugging (3.16) into (3.15), by Cauchy's inequality and the fact that $|\delta u| \leq|D u|$, we then have at $x_{0}$,

$$
\begin{aligned}
D_{\beta} v & \geq-C\left(1+|D u|^{2}\right)+M_{1}^{2}[\kappa-\alpha K \eta|\varphi|(\cdot, u)] \\
& \geq \frac{1}{2} \kappa M_{1}^{2}-C,
\end{aligned}
$$


provided $\kappa$ is chosen large enough so that $\kappa \geq C\left(1+\alpha K \sup _{\partial \Omega} \eta\right)$, where $C$ is a constant depending on $\beta, \varphi, \Omega$ and $M_{0}$. With the constant $\kappa$ fixed, from (3.17) and $D_{\beta} v\left(x_{0}\right) \leq 0$, we obtain

$$
M_{1}^{2} \leq \frac{2 C}{\kappa}
$$

We next consider the case that the maximum of $v$ occurs at a point $x_{0} \in \Omega$. We now take the constant $\alpha$ sufficiently small and fix the defining function $\phi$ such that

$$
\alpha \leq a_{1} / 16 \sup _{\Omega} \eta, \quad \phi \geq-a_{1} / 16 \kappa \text { in } \Omega .
$$

Taking (3.12) into account, these restrictions in (3.19) will enable us to proceed from estimating $D u\left(x_{0}\right)$ to an estimate for $M_{1}$. Note that these conditions ultimately depend on the independent choice of the constant $K$. Since $D v\left(x_{0}\right)=0$ and $D^{2} v\left(x_{0}\right) \leq 0$, we have

$$
\mathcal{L} v=F^{i j} D_{i j} v-\left(F^{i j} A_{i j}^{k}+D_{p_{k}} B\right) D_{k} v=F^{i j} D_{i j} v \leq 0, \quad \text { at } x_{0},
$$

where $\mathcal{L}$ is the linearized operator defined in (2.5). Our estimations then reduce to getting an appropriate lower bound for $\mathcal{L} \eta$ and for this we separate cases (i) and (ii) in Theorem 1.3.

Case (i): A uniformly regular.

Here we take the "+" sign in $\eta$, that is $\eta=e^{K\left(u_{0}-u\right)}$, and for convenience set $u_{0}=M_{0}^{+}=\sup _{\Omega} u$ so that $\eta \geq 1$. Our estimation of $L \eta$ is motivated by the barrier constructions in $[11,13,17,18]$ for regular $A$, where the constant $u_{0}$ is replaced by an admissible function $\bar{u}$. In particular the reader is referred to Section 2 in [13] for the extension to general operators. First by Taylor's formula, we have

$$
\begin{aligned}
\mathcal{L}\left(u_{0}-u\right)= & F^{i j}\left[-w_{i j}-A_{i j}(\cdot, u, 0)\right. \\
& \left.+A_{i j}(\cdot, u, 0)-A_{i j}(\cdot, u, D u)+A_{i j}^{k}(\cdot, u, D u) u_{k}\right]+\left(D_{p_{k}} B\right) u_{k} \\
= & \frac{1}{2} F^{i j} A_{i j}^{k l}(\cdot, u, \hat{p}) u_{k} u_{l}-F^{i j}\left(w_{i j}+A_{i j}(\cdot, u, 0)\right)+\left(D_{p_{k}} B\right) u_{k},
\end{aligned}
$$

where $\hat{p}=\theta D u$ with $\theta \in(0,1)$. Since $A$ is uniformly regular, we can estimate

$$
\frac{1}{2} F^{i j} A_{i j}^{k l}(\cdot, u, \hat{p}) u_{k} u_{l} \geq \frac{\lambda_{0}}{2}|D u|^{2} \mathscr{T}-\frac{\bar{\lambda}_{0}}{2} F^{i j} u_{i} u_{j} .
$$

From (3.21), (3.22), we have

$$
\begin{aligned}
\mathcal{L} \eta & =K \eta\left[\mathcal{L}\left(u_{0}-u\right)+K F^{i j} u_{i} u_{j}\right] \\
& \geq K \eta\left[\frac{\lambda_{0}}{2}|D u|^{2} \mathscr{T}+\left(K-\frac{\bar{\lambda}_{0}}{2}\right) F^{i j} u_{i} u_{j}\right.
\end{aligned}
$$




$$
\begin{aligned}
& \left.-F^{i j}\left(w_{i j}+A_{i j}(\cdot, u, 0)\right)+\left(D_{p_{k}} B\right) u_{k}\right] \\
\geq & K \eta\left[\frac{\lambda_{0}}{2}|D u|^{2} \mathscr{T}+\frac{K}{2} F^{i j} u_{i} u_{j}-F^{i j} w_{i j}-\mu \mathscr{T}+\left(D_{p_{k}} B\right) u_{k}\right],
\end{aligned}
$$

by choosing $K \geq \bar{\lambda}_{0}$, where $\mu$ is a constant depending on $A$. At this point we introduce a more general condition than the concavity F2 which also includes the homogeneous case. Namely we assume for any constant $a>a_{0}$, there exist non-negative constants $\mu_{0}$ and $\mu_{1}$ such that

$$
r \cdot F_{r} \leq F+\mu_{0}+\mu_{1} \mathscr{T}
$$

whenever $F \geq a$. From (1.9), we see that F2 implies (3.24), with $\mu_{0}=$ $\max \{0,-F(\mu I)\}$ and $\mu_{1}=\mu$ for any $\mu>0$. Note that when $a_{0}>-\infty$, then (3.24), with $\mu_{0}=\max \left\{0,-a_{0}\right\}$ and $\mu_{1}=0$, is immediate from (1.10). Using (3.24) in (3.23) we thus obtain

$$
\mathcal{L} \eta \geq K \eta\left[\frac{\lambda_{0}}{2}|D u|^{2} \mathscr{T}+\frac{K}{2} F^{i j} u_{i} u_{j}-\mu(1+\mathscr{T})-B+\left(D_{p_{k}} B\right) u_{k}\right],
$$

where now $\mu$ depends on $A, \mu_{0}$ and $\mu_{1}$.

Assuming now F5, with $b=\infty$, so that $\mathscr{T} \geq \delta_{0}$ for $B \geq b_{0}>a_{0}$ and supplementing the growth conditions (3.4) by

$$
B-p \cdot B_{p} \leq o\left(|p|^{2}\right),
$$

we then have from (3.25), with $\omega$ sufficiently small,

$$
\begin{aligned}
\mathcal{L} \eta & \geq K \eta \mathscr{T}\left[\frac{\lambda_{0}}{2}|D u|^{2}-\omega|D u|^{2}-C\right] \\
& \geq \frac{K \lambda_{0}}{4} \mathscr{T}|D u|^{2}
\end{aligned}
$$

provided $|D u| \geq C_{1}$ for some sufficiently large constant $C_{1}$, depending on $F, A, B$ and $M_{0}$. Combining (3.9), (3.27), and also choosing $K \geq \frac{4}{\lambda_{0}}$, we then obtain

$$
\mathcal{L} v \geq \alpha M_{1}^{2}|D u|^{2} \mathscr{T}-C \mathscr{T}\left(\omega|D u|^{4}+\kappa M_{1}^{2} \omega|D u|^{2}+1\right) .
$$

On the other hand if $\mathrm{F}^{+}$is satisfied, with $b=\infty$, and " $O$ " is weakened to " $O$ " in (3.26), that is

$$
B-p \cdot B_{p} \leq O\left(|p|^{2}\right)
$$

then we have from (3.25),

$$
\mathcal{L} \eta \geq K \eta\left[\left(\frac{\lambda_{0}}{2}|D u|^{2}-\mu\right) \mathscr{T}+\frac{K}{2} F^{i j} u_{i} u_{j}-C\left(1+|D u|^{2}\right)\right],
$$


and we arrive again at the inequality (3.28), with large enough $K$, by using the positivity of $F_{r}$ to estimate

$$
F^{i j} u_{i} u_{j} \geq \delta_{1}|D u|^{2}
$$

for some positive constant $\delta_{1}$ when $|r| \leq C$ and $F(r) \geq b_{0}$.

Now since $v$ is maximised at $x_{0}$ and $\omega$ can be made arbitrarily small for large enough $|D u|$, we must have $\left|D u\left(x_{0}\right)\right| \leq C$, noting that $\alpha$ and $\kappa$ are now fixed by our choice of $K$ above. From (3.12), (3.13) and our restrictions (3.19) on $\alpha$ and $\phi$, we obtain

$$
v \leq C+\frac{a_{1}}{8} M_{1}^{2}, \quad \text { in } \Omega,
$$

so that again using (3.12), we conclude $M_{1} \leq C$, where $C$ now depends on $F, A, B, \Omega, b_{0}, \beta, \varphi$ and $M_{0}$. This completes the proof of case (i) in Theorem 1.3, with conditions (1.22) and F2 weakened to (3.4) and (3.24), and also with (3.26) weakened to (3.29) if $\mathrm{F}^{+}$is satisfied, with $b=\infty$. Note though that this latter condition is incompatible with $\mathrm{F} 2$.

Case (ii): F7 holds, $\beta=v$.

We take the "-" sign in $\eta$ so that $\eta=e^{K\left(u-u_{0}\right)}$ and set $u_{0}=M_{0}^{-}=\inf _{\Omega} u$. Assuming growth conditions,

$$
p \cdot A_{p} \leq O\left(|p|^{2}\right) I, \quad p \cdot B_{p} \leq O\left(|p|^{2}\right),
$$

as $|p| \rightarrow \infty$, uniformly for $x \in \Omega,|z| \leq M$ for any $M>0$, we then have from (3.6), in place of (3.23),

$$
\begin{aligned}
\mathcal{L} \eta & =K \eta\left[\mathcal{L}\left(u-u_{0}\right)+K F^{i j} u_{i} u_{j}\right] \\
& =K \eta\left[K F^{i j} u_{i} u_{j}+F^{i j} u_{i j}-\left(F^{i j} A_{i j}^{k}+B_{p_{k}}\right) u_{k}\right] \\
& \geq K \eta\left[K F^{i j} u_{i} u_{j}+F^{i j} u_{i j}-\mu(\mathscr{T}+1)\left(1+|D u|^{2}\right)\right],
\end{aligned}
$$

where $\mu$ is a constant depending on $M_{0}$. At this stage, anticipating our use of F7, we can fix the constant $K$ so that $K \geq 2 n(1+2 \mu) / \min \left\{\delta_{0}, \delta_{1}\right\}$. In order to get a lower bound for $\mathcal{L} \eta$, similar to (3.27), at the point $x_{0} \in \Omega$ where $v$, given by (3.13), is maximised, we also need to impose our key restrictions in the hypotheses of case (ii) of Theorem 1.3 , namely $\mathcal{F}$ is orthogonally invariant, $\beta=v$, that is $\beta^{\prime}=0$, and

$$
A=o\left(|p|^{2}\right)
$$

as $|p| \rightarrow \infty$, uniformly for $x \in \Omega,|z| \leq M$ for any $M>0$.

By choosing coordinates, we can assume the augmented Hessian $M[u]=D^{2} u-$ $A=\left\{w_{i j}\right\}$ is diagonal at $x_{0}$. Then we have, at $x_{0}$,

$$
u_{i i}=w_{i i}+A_{i i}, \quad \forall i, \quad \text { and } \quad u_{i j}=A_{i j}, \quad \text { for } i \neq j .
$$

From now on, all the calculations will be made at the maximum point $x_{0}$. Since $D v\left(x_{0}\right)=0$, we have 


$$
v_{i}=g_{i}+M_{1}^{2}\left(\alpha K \eta u_{i}-\kappa \phi_{i}\right)=0, \text { for } i=1, \ldots, n,
$$

that is

$$
g_{i}=M_{1}^{2}\left(-\alpha K \eta u_{i}+\kappa \phi_{i}\right), \text { for } i=1, \ldots, n \text {. }
$$

On the other hand, we have

$$
\begin{aligned}
g_{i} & =D_{i}\left[|\delta u|^{2}+\left(D_{\nu} u-\varphi\right)^{2}\right] \\
& =D_{i}\left[|D u|^{2}-2 \varphi D_{\nu} u+\varphi^{2}\right] \\
& =2\left(u_{k}-\varphi v_{k}\right) u_{k i}-2 \varphi u_{k} D_{i} v_{k}+2\left(\varphi-D_{\nu} u\right) \tilde{D}_{x_{i}} \varphi,
\end{aligned}
$$

for $i=1, \ldots, n$, where $\tilde{D}_{x}=D_{x}+D u D_{z}$ and in accordance with our extension of $G$ to $\Omega, \varphi=0$ for $d \geq \rho$. Combining (3.36), (3.37) and (3.34), we have

$$
\begin{aligned}
\left(u_{i}-\varphi v_{i}\right) w_{i i}= & \frac{1}{2} M_{1}^{2}\left(-\alpha K \eta u_{i}+\kappa \phi_{i}\right)+\varphi u_{k} D_{i} v_{k}-\left(\varphi-D_{\nu} u\right) \tilde{D}_{x_{i}} \varphi \\
& -\left(u_{i}-\varphi v_{i}\right) A_{i i}-\sum_{k \neq i}\left(u_{k}-\varphi v_{k}\right) A_{k i},
\end{aligned}
$$

for $i=1, \ldots, n$. Without loss of generality, we can further choose our coordinates so that

$$
u_{1}\left(x_{0}\right) \geq \frac{1}{\sqrt{n}}\left|D u\left(x_{0}\right)\right| .
$$

By assuming

$$
u_{1}\left(x_{0}\right)>2 \max \left\{\sup _{\Omega}|\varphi|, \kappa \sup _{\Omega}(|D \phi| / \alpha K)\right\},
$$

we also have

$$
\frac{u_{1}}{u_{1}-\varphi v_{1}} \geq \frac{2}{3},
$$

at $x_{0}$. From (3.38), (3.40) and (3.41), we then obtain

$$
\begin{aligned}
w_{11}= & \frac{1}{u_{1}-\varphi v_{1}}\left[\frac{1}{2} M_{1}^{2}\left(-\alpha K \eta u_{1}+\kappa \phi_{1}\right)+\varphi u_{k} D_{1} v_{k}\right. \\
& \left.-\left(\varphi-D_{\nu} u\right) \tilde{D}_{x_{1}} \varphi-\sum_{k>1}\left(u_{k}-\varphi v_{k}\right) A_{k 1}\right]-A_{11} \\
\leq & \frac{1}{u_{1}-\varphi v_{1}}\left[-\frac{1}{4} \alpha K \eta M_{1}^{2} u_{1}+\left|\varphi u_{k} D_{1} v_{k}\right|\right. \\
& \left.+\left|\left(\varphi-D_{\nu} u\right) \tilde{D}_{x_{1}} \varphi\right|+\left|\sum_{k>1}\left(u_{k}-\varphi v_{k}\right) A_{k 1}\right|\right]+\left|A_{11}\right|
\end{aligned}
$$




$$
\begin{aligned}
& \leq-\frac{1}{6} \alpha K \eta M_{1}^{2}+C\left(\left|\varphi D_{1} v_{k}\right|+\left|\tilde{D}_{x_{1}} \varphi\right|+\sum_{k>1}\left|A_{k 1}\right|\right)+\left|A_{11}\right| \\
& \leq-\frac{1}{6} \alpha K M_{1}^{2}+C\left(\omega|D u|^{2}+1\right),
\end{aligned}
$$

at $x_{0}$, where $\omega=\omega(|D u|)$ approaches 0 as $|D u| \rightarrow \infty$ and the growth condition (3.33) is used in the last inequality. It then follows that $w_{11}\left(x_{0}\right)<0$ provided $\left|D u\left(x_{0}\right)\right| \geq C_{1}$ for some constant $C_{1}$, depending on $F, A, B, \Omega, \varphi$ and $M_{0}$. Since $w_{11}$ is the eigenvalue of $M[u]$ corresponding to the eigenvector $e_{1}$ and the matrix $F_{r}$ is diagonal at $M[u]$, by virtue of the orthogonal invariance of $\mathcal{F}$, we then obtain from F7 and (3.39) that

$$
F^{i j} u_{i} u_{j} \geq F^{11} u_{1}^{2} \geq \frac{1}{n}\left(\delta_{0}+\delta_{1} \mathscr{T}\right)|D u|^{2}
$$

at $x_{0}$. From our choice of $K$ we then obtain, from (3.32) and (3.43),

$$
\mathcal{L} \eta \geq K \eta\left[2(1+\mathscr{T})|D u|^{2}+F^{i j} u_{i j}\right],
$$

at $x_{0}$. Note that if $F^{i j} w_{i j} \geq 0$, as in (1.10), then we can absorb the term $F^{i j} u_{i j}$ in the last term in (3.32) so that it is not present in (3.44). Furthermore if $F$ is positive homogeneous of degree one, we can replace $p \cdot B_{p}$ in (3.31) by $p \cdot B_{p}-B$.

Assuming also the growth conditions (3.4), we now combine (3.44) with (3.9), with $a_{1}=1$, and in general, (when $F^{i j} w_{i j}$ may be unbounded from below), use Cauchy's inequality to control the term $F^{i j} u_{i j}$. Accordingly we obtain, at the maximum point $x_{0}$,

$$
\begin{aligned}
0 \geq \mathcal{L} v \geq & \alpha M_{1}^{2} K\left[2(1+\mathscr{T})|D u|^{2}-\alpha M_{1}^{2} K \eta \mathscr{T}\right] \\
& -C(1+\mathscr{T})\left(\omega|D u|^{4}+\kappa M_{1}^{2} \omega|D u|^{2}+1\right) \\
\geq & (1+\mathscr{T})\left[\alpha M_{1}^{2}|D u|^{2}-C\left(\omega|D u|^{4}+\kappa M_{1}^{2} \omega|D u|^{2}+1\right)\right]
\end{aligned}
$$

provided, taking account of (3.12) and (3.13), we further restrict $\alpha$ so that $\alpha K \eta \leq \frac{1}{16}$. As in case (i), since $\omega$ can be made arbitrarily small for large enough $|D u|$ and, $\alpha$ and $\kappa$ are fixed by our choice of $K$ above, we obtain an estimate $\left|D u\left(x_{0}\right)\right| \leq C$ and hence $M_{1} \leq C$, where $C$ depends on $F, A, B, \Omega, b_{0}, \varphi$ and $M_{0}$. This completes the proof of case (ii) in Theorem 1.3, with conditions (1.22) weakened to (3.4) and (3.31).

Remark 3.1 When $\beta=v$, so that $a_{i j}=\delta_{i j}$ in (3.3), we can further weaken the general growth conditions (3.4), in both cases of the above proof, to

$$
\begin{aligned}
& p \cdot D_{x} A+|p|^{2} D_{z} A \geq o\left(|p|^{4}\right) I, \quad p \cdot D_{x} B+|p|^{2} D_{z} B \geq o\left(|p|^{4}\right) \\
& D_{x} A, D_{x} B=o\left(|p|^{4}\right), \quad D_{z} A, D_{z} B=o\left(|p|^{3}\right), \quad D_{p} A, D_{p} B=o\left(|p|^{3}\right)
\end{aligned}
$$

as $|p| \rightarrow \infty$, uniformly for $x \in \Omega,|z| \leq M$ for any $M>0$, while if also $\varphi=0$ so that $g=|D u|^{2}$, we need only assume, in place of (3.4),

$$
p \cdot D_{x} A+|p|^{2} D_{z} A \geq o\left(|p|^{4}\right) I, \quad p \cdot D_{x} B+|p|^{2} D_{z} B \geq o\left(|p|^{4}\right) .
$$


Discarding the boundary condition (3.2) so that $g=|D u|^{2}$ and $\kappa=0$ in (3.13), we then have a global gradient estimate for admissible solutions $u \in C^{3}(\Omega) \cap C^{1}(\bar{\Omega})$ in terms of the gradient on the boundary, namely

$$
\sup _{\Omega}|D u| \leq C\left(1+\sup _{\partial \Omega}|D u|\right),
$$

where $C$ is a constant depending on $F, A, B, \Omega, b_{0}$ and $|u|_{0 ; \Omega}$. The estimate (3.48) thus holds when $F, A$ and $B$ satisfy the hypotheses of Theorem 1.3, but more generally we can replace (1.22) by (3.47) with cases (i) and (ii) replaced respectively by

(i') $A$ is uniformly regular, $F$ satisfies (3.24) and either (a) F5, with $b=\infty$, and (3.26), or (b) $\mathrm{F}^{+}$, with $b=\infty$ and (3.29);

(ii') $\mathcal{F}$ is orthogonally invariant satisfying F7, $A$ and $B$ satisfy (3.31) and (3.33).

Using our barrier constructions in Section 2 of [13] in the proof of case (i') also enables some alternative conditions to uniform regularity which would include the case when $A$ is independent of $p$.

Remark 3.2 From the estimate (3.48) in Remark 3.1, we can infer, under the same hypotheses, a global gradient bound for admissible solutions $u \in C^{3}(\Omega) \cap C^{1}(\bar{\Omega})$ of the boundary value problem (1.1)-(1.2) for nonlinear $\mathcal{G}$, when $G$ is uniformly concave in $p$, that is

$$
D_{p_{i} p_{j}} G(x, z, p) \xi_{i} \xi_{j} \leq-\sigma_{0}|\xi|^{2}
$$

for all $x \in \partial \Omega,|z| \leq M, p \in \mathbb{R}^{n}$, any unit vector $\xi$, and some positive constant $\sigma_{0}$, depending on the constant $M$. By virtue of the global bound (3.48), we only need to estimate $D u$ on $\partial \Omega$. Using Taylor's expansion, with $\theta \in(0,1)$, we have on $\partial \Omega$,

$$
\begin{aligned}
0=G(x, u, D u)= & G(x, u, 0)+D_{p_{i}} G(x, u, 0) D_{i} u \\
& +\frac{1}{2} D_{p_{i} p_{j}} G(x, u, \theta D u) D_{i} u D_{j} u \\
\leq & G(x, u, 0)+D_{p_{i}} G(x, u, 0) D_{i} u-\frac{1}{2} \sigma_{0}|D u|^{2},
\end{aligned}
$$

which leads to

$$
|D u| \leq C, \quad \text { on } \partial \Omega,
$$

and hence

$$
|D u| \leq C, \quad \text { on } \Omega,
$$

where $C$ depends on $F, A, B, \Omega, b_{0}, \sigma_{0}$ and $|u|_{0 ; \Omega}$. 
Remark 3.3 We remark that it is not necessary to restrict $\beta=v$ in case (ii) of Theorem 1.3 and we can assume more generally

$$
|\beta-v|=\left|\beta^{\prime}\right|<1 / \sqrt{n} \text {. }
$$

Replacing $v$ by $\beta$ in (3.37), we still obtain $w_{11}\left(x_{0}\right)<0$, if $\left|D u\left(x_{0}\right)\right| \geq C_{1}$, under condition (3.53), where now $C_{1}$ depends also on $\sup _{\partial \Omega}|\beta-v|$.

\subsection{Local gradient estimates}

In this subsection we prove local and interior versions of Theorem 1.3 and unlike the global gradient estimates in the previous section we will need the full strength of the growth conditions in (1.22) with respect to the $p$ variables. The local estimates will also provide us with estimates in terms of moduli of continuity of solutions under weaker growth conditions analogous to the uniformly elliptic case in [25]. For the latter estimates we also need to assume in case (ii) a complementary condition to (3.24), namely that there exist non-negative constants $\mu_{0}, \mu_{1}$ and $\mu_{2}$ such that for any $r \in \Gamma$,

$$
-r \cdot F_{r} \leq \mu_{0}+\mu_{1} \mathscr{T}(r)+\mu_{2}|F(r)|
$$

Clearly (3.54) is satisfied trivially for positive homogeneous $F$ or if F2 and either $a_{0}>-\infty$ or F4 are satisfied by (1.10).

We summarise the results in the following theorem, where for convenience we use balls rather than the domains $\Omega_{0}$ and $\Omega^{\prime}$ in Theorem 1.1.

Theorem 3.1 Let $u \in C^{3}(\Omega)$ be an admissible solution of equation (1.1) in $\Omega$ and assume that $\mathcal{F}$ satisfies $F 1$ and $F 3, A, B \in C^{1}\left(\bar{\Omega} \times \mathbb{R} \times \mathbb{R}^{n}\right)$, satisfy (1.22), $b_{0}:=$ $\inf _{\Omega} B(\cdot, u, D u)>a_{0}$ together with one of the following further conditions:

(i) A is uniformly regular, $F$ satisfies (3.24) and either (a)F5, with $b=\infty$, and (3.26), or (b) $F 5^{+}$, with $b=\infty$;

(ii) $\mathcal{F}$ is orthogonally invariant satisfying $F 7$ and A satisfies (3.33).

Then for any $y \in \bar{\Omega}, 0<R<1$ and ball $B_{R}=B_{R}(y)$, we have the estimate

$$
|D u(y)| \leq C\left(\frac{1}{R}+\sup _{\partial \Omega \cap B_{R}}|D u|\right),
$$

for $u \in C^{1}\left(\bar{\Omega} \cap B_{R}\right)$, where $C$ is a constant depending on $F, A, B, \Omega, b_{0}$ and $|u|_{0 ; \Omega \cap B_{R}}$. Furthermore if $y \in \partial \Omega, \partial \Omega \cap B_{R} \in C^{3}$ and $\mathcal{G}[u]=0$ on $\partial \Omega$, for an oblique semilinear boundary operator $\mathcal{G}$, given by (1.23), with $\varphi \in C^{2}\left(\partial \Omega \cap B_{R} \times \mathbb{R}\right)$, $\beta \in C^{2}\left(\partial \Omega \cap B_{R}\right)$ in case (i) and $\beta=v$ in case (ii), then we have the estimate

$$
|D u(y)| \leq \frac{C}{R}
$$


for $u \in C^{2}\left(\bar{\Omega} \cap B_{R}\right)$, where $C$ depends additionally on $\beta$ and $\varphi$. If " $o$ " is extended to " $O$ " in (1.22) and (3.33), then there exists a positive constant $\theta$ depending on the same quantities as $C$, such that the estimates (3.55) and (3.56) continue to hold provided osc $u<\theta$ and $\mathcal{F}$ satisfies (3.54) in case (ii) with $C$ depending additionally on $\mu_{0}$, $\Omega \cap B_{R}$ $\mu_{1}$ and $\mu_{2}$.

Proof Theorem 3.1 follows by modification of the proof of Theorem 1.3. First we fix a function $\zeta \in C^{0}\left(\bar{B}_{R}\right) \cap C^{2}(S)$, satisfying $0 \leq \zeta \leq 1$, where $S=S_{\zeta} \subset B_{R}$, denotes the support of $\zeta$. From (1.22), (3.9) and Cauchy's inequality, we now obtain in place of (3.9),

$$
\begin{aligned}
\mathcal{L}\left(\zeta^{2} g\right)= & \zeta^{2} \mathcal{L} g+\left(\mathcal{L} \zeta^{2}\right) g+2 F^{i j} D_{i} \zeta^{2} D_{j} g \\
\geq & a_{1} \zeta^{2} \mathcal{E}_{2}^{\prime}-C \zeta^{2}(\mathscr{T}+1)\left(\omega|D u|^{4}+1\right)-C(\mathscr{T}+1)\left(|D u|^{2}+1\right)\left[|D \zeta|^{2}\right. \\
& \left.+(|D u|+1) \zeta|D \zeta|+\zeta\left|D^{2} \zeta\right|\right] \\
\geq & a_{1} \zeta^{2} \mathcal{E}_{2}^{\prime}-C(\mathscr{T}+1)\left(|D u|^{2}+1\right)\left(\omega \zeta^{2}|D u|^{2}+\zeta|D u||D \zeta|\right. \\
& \left.+|D \zeta|^{2}+\left|D^{2} \zeta\right|+1\right)
\end{aligned}
$$

in $\Omega \cap S$, where $\omega=\omega(|D u|)$ is a positive decreasing function on $[0, \infty)$ tending to 0 at infinity. With $g$ defined by (3.10), we consider now in place of (3.13), auxiliary functions of the form

$$
v:=\zeta^{2} g+\tilde{M}_{1}^{2}(\alpha \eta-\kappa \phi)
$$

where $\tilde{M}_{1}=\sup _{\Omega \cap B_{R}} \zeta|D u|, \alpha, \kappa, \eta$ and $\phi$ are as before, except that $\Omega$ is replaced by $\Omega \cap B_{R}$, in the definitions of $M_{0}^{+}$and $M_{0}^{-}$. For the estimate (3.55), which is the local version of the estimate (3.48) in Remark 3.1, we take as there $g=|D u|^{2}, \kappa=0$ and choose

$$
\zeta(x)=1-\frac{|x-y|^{2}}{R^{2}}
$$

so that

$$
\zeta(y)=1,|D \zeta| \leq c / R,\left|D^{2} \zeta\right| \leq c / R^{2},
$$

for some constant $c$, and

$$
\mathcal{L}\left(\zeta^{2} g\right) \geq a_{1} \zeta^{2} \mathcal{E}_{2}^{\prime}-C(\mathscr{T}+1)\left(\omega \zeta^{2}|D u|^{4}+\frac{1}{R} \zeta|D u|^{3}\right)
$$

at the maximum point $x_{0}$ of $v$ in $\Omega \cap S$, provided $\zeta\left(x_{0}\right)\left|D u\left(x_{0}\right)\right|>1 / R$ and $\left|D u\left(x_{0}\right)\right|>$ 1. For the estimate (3.56), we need to first take $R$ sufficiently small so that there exists a cut-off function $\zeta \in C^{0}\left(\bar{B}_{R}\right) \cap C^{2}\left(S_{\zeta}\right), 0 \leq \zeta \leq 1$ satisfying (3.60) together with the boundary condition 


$$
D_{\beta} \zeta=0 \text { on } \partial \Omega \cap S_{\zeta}
$$

We show how to construct such a function $\zeta$ from the function (3.59) at the end of the proof.

From the property (3.62), we now obtain in place of (3.15),

$$
\begin{aligned}
D_{\beta} v & =2 \zeta\left(D_{\beta} \zeta\right) g+D_{\beta}|\delta u|^{2}+\tilde{M}_{1}^{2}\left(\alpha D_{\beta} \eta-\kappa D_{\beta} \phi\right) \\
& \geq 2 \zeta^{2}\left(\delta_{k} u\right) D_{\beta} \delta_{k} u+\kappa \tilde{M}_{1}^{2}-\alpha K \eta|\varphi|(\cdot, u) \tilde{M}_{1}^{2},
\end{aligned}
$$

on $\partial \Omega \cap S$. With these modifications, the estimates (3.55) and (3.56) follow from the proof of Theorem 1.3, with $M_{1}$ replaced by $\tilde{M}_{1}$. In case (ii), we obtain in place of the estimate (3.42),

$$
\zeta^{2} w_{11} \leq-\frac{1}{6} \alpha K M_{1}^{2}+C\left[\zeta^{2}\left(\omega|D u|^{2}+1\right)+\frac{1}{R} \zeta|D u|\right]
$$

so that we obtain again $w_{11}\left(x_{0}\right)<0$ provided $\zeta\left(x_{0}\right)\left|D u\left(x_{0}\right)\right|>C_{1} / R$, for some constant $C_{1}$, depending on $F, A, B, \Omega, \varphi$ and $M_{0}$.

If we replace " $O$ " by " $O$ " in the structure conditions (1.22), we obtain (3.61) with $\omega=1$. Similarly if we replace " $O$ " by " $O$ " in (3.33), we obtain (3.64) with $\omega=1$. Accordingly we may still arrive at our desired gradient estimates, (3.55) and (3.56), if $\alpha$ can be chosen sufficiently large, in which case we can still satisfy (3.19) for $\alpha$, provided $\eta$ is replaced by $\eta-1$ in (3.58) and

$$
\underset{\Omega \cap B_{R}}{\operatorname{osc}} u=M_{0}^{+}-M_{0}^{-} \leq \theta:=\frac{1}{K} \log \left(1+\frac{a_{1}}{16 \alpha}\right) .
$$

Note that in case (ii), we cannot satisfy the further restriction $\alpha K \eta \leq \frac{1}{16}$, for large $\alpha$, so here we use condition (3.54) to control the term $F^{i j} u_{i j}$ in (3.44). We remark that when $A$ is regular such a control can be alternatively achieved through a barrier [13].

To end the proof of Theorem 3.1, we give the key construction of the cut-off function at boundary.

Construction of cut-off function at boundary. We fix a point $y \in \partial \Omega$, which we may take to be the origin, and a coordinate system so that $v(0)=e_{n}$. Suppose that in some ball $B_{\rho}=B_{\rho}(0), \Omega \cap B_{\rho}=\left\{x_{n}>h\left(x^{\prime}\right)\right\}, \partial \Omega \cap B_{\rho}=\left\{x_{n}=h\left(x^{\prime}\right)\right\}$, where $h \in C^{3}\left(\bar{B}_{\rho}^{\prime}\right), B_{\rho}^{\prime}=\left\{\left|x^{\prime}\right|<\rho\right\}$ and $x^{\prime}=\left(x_{1}, \ldots, x_{n-1}\right)$. By taking $\rho$ sufficiently small, we can assume,

$$
\chi(x):=\beta_{n}(x)-\sum_{i=1}^{n-1} \beta_{i}(x) D_{i} h\left(x^{\prime}\right) \geq 1-\delta
$$

for any fixed $\delta>0$, since $\beta_{n}(0)=1, D h(0)=0$. Now we consider a coordinate transformation $x \rightarrow \tilde{x}=\psi(x)$, where 


$$
\begin{aligned}
\tilde{x}_{i} & =x_{i}-\beta_{i}(x) \tilde{x}_{n}, \quad i=1, \ldots, n-1, \\
\tilde{x}_{n} & =\frac{1}{\chi(x)}\left(x_{n}-h\left(x^{\prime}\right)\right) .
\end{aligned}
$$

Again with $\rho$ sufficiently small, we have $\psi(0)=0, \psi\left(\partial \Omega \cap B_{\rho}\right)=\left\{\tilde{x}_{n}=0\right\}$, $D \psi(0)=I$ and det $D \psi>0$, so that in particular $\psi$ is invertible in $B_{\rho}$. Furthermore, if $\zeta \in C^{1}\left(\bar{\Omega} \cap B_{\rho}\right), \tilde{\zeta}=\zeta \circ \psi^{-1} \in C^{1}\left(\psi\left(\bar{\Omega} \cap B_{\rho}\right)\right)$, we obtain by calculation

$$
\begin{aligned}
D_{\tilde{x}_{n}} \tilde{\zeta} \circ \psi & =\sum_{i=1}^{n} \frac{\partial \zeta}{\partial x_{i}} \frac{\partial x_{i}}{\partial \tilde{x}_{n}} \\
& =\sum_{i=1}^{n-1} \beta_{i}(x) \frac{\partial \zeta}{\partial x_{i}}+\left(\chi(x)+\sum_{i=1}^{n-1} \beta_{i}(x) D_{i} h\left(x^{\prime}\right)\right) \frac{\partial \zeta}{\partial x_{n}} \\
& =\sum_{i=1}^{n-1} \beta_{i}(x) \frac{\partial \zeta}{\partial x_{i}}+\beta_{n}(x) \frac{\partial \zeta}{\partial x_{n}}=D_{\beta} \zeta, \quad \text { on } \partial \Omega \cap B_{\rho} .
\end{aligned}
$$

Hence if $\tilde{\zeta} \in C_{0}^{1}\left(\psi\left(\bar{\Omega} \cap B_{\rho}\right)\right)$ satisfies $D_{\tilde{x}_{n}} \tilde{\zeta}\left(\tilde{x}^{\prime}, 0\right)=0$, we have $D_{\beta} \zeta=0$ on $\partial \Omega \cap B_{\rho}$. With the help of (3.68), a boundary cut-off function $\zeta \in C^{0}\left(\bar{B}_{R}\right) \cap C^{2}\left(S_{\zeta}\right), 0 \leq$ $\zeta \leq 1$ satisfying (3.60) and (3.62) can be constructed. For a fixed point $y \in \partial \Omega$, which we may take to be the origin, we make the coordinate transformation $x \rightarrow \tilde{x}=\psi(x)$ as in (3.67). In the $\tilde{x}$-coordinate system, we can choose the function

$$
\tilde{\zeta}=1-\frac{|\tilde{x}|^{2}}{\tilde{R}^{2}},
$$

in $B_{\tilde{R}} \subset \psi\left(B_{\rho}\right)$, with $\tilde{\zeta}$ vanishing outside $B_{\tilde{R}}$. Then for $R=C \tilde{R} \leq \rho$, where $C$ depends on $\Omega$ and $\beta$, the function $\zeta=\tilde{\zeta} \circ \psi$ is the desired cut-off function satisfying the above properties (3.60) and (3.62). The estimate (3.56) for all $0<R<1$, then follows immediately.

Remark 3.4 Note that when $\beta=v$, (3.10) is similar to the corresponding function used for the gradient estimate of Neumann problems in [19], (and more recently for the $k$-Hessian equations in [30]). In our proof, we use the auxiliary functions (3.13) and (3.58), which are modifications of the auxiliary functions used in Section 3 of [25] for uniformly elliptic equations and for interior gradient bounds for $k$-Hessian equations in [39]. We remark that we can use alternative functions; in particular functions of the form $v=g \exp (\alpha \eta-\kappa \phi)$ and $v=\zeta^{2} g \exp (\alpha \eta-\kappa \phi)$, with appropriately chosen positive constants $\alpha$ and $\kappa$, in place of (3.13) and (3.58) respectively.

Remark 3.5 If we assume the matrix $A$ satisfies the following condition

$$
D_{z} A(x, z, p) \geq c|p|^{2} I
$$

as $|p| \rightarrow \infty, x \in \Omega,|z| \leq M, p \in \mathbb{R}^{n}$ for any $M>0$ and for some $c>0$, we can dispense with the uniformly regular assumption on $A$ in Theorem 1.3 and Theorem 3.1 and the proof is much simpler. More generally we can replace the exponent 2 on the 
right hand side of (3.70) by $m$ for $0 \leq m \leq 2$ provided the powers of $|p|$ in the growth conditions (1.22) are reduced by $2-m$. When the constant $c$ is sufficiently large, " $o$ " in (1.22) can be weakened to " $O$ ".

Remark 3.6 Using the cut-off function $\zeta$ constructed in the proof of Theorem 3.1, we can also extend the global second derivative estimates in Theorem 1.2 to local estimates in the case of semilinear boundary operators $\mathcal{G}$. As in Remark 2.1 we need only assume $\partial \Omega \cap B$ is uniformly $(\Gamma, A, G$ )-convex with respect to $u$ for some ball $B=B_{R}(y)$ of radius $R$, centred at $y \in \bar{\Omega}$. Under the hypotheses of Theorem 1.2, with $\Omega$ replaced by $\Omega \cap B$ and $\partial \Omega$ replaced by $\partial \Omega \cap B$, we then obtain for semilinear $\mathcal{G}$, in place of (1.18),

$$
\left|D^{2} u(y)\right| \leq C\left(1+R^{-2}\right)
$$

where $C$ is now a constant depending on $F, A, B, G, \Omega, \beta_{0}$ and $|u|_{1 ; \Omega \cap B}$. A more detailed argument is presented in [15], where we consider local boundary regularity for appropriate classes of weak solutions.

Remark 3.7 We remark that the uniformly regular definition (1.20) (1.21) of the matrix $A$, can also be equivalently formulated as follows, namely

$$
\lambda(x, u, p)=\inf _{\substack{|\xi|=|\eta|=1, \xi \cdot \eta=0}} A_{i j}^{k l}(x, u, p) \xi_{i} \xi_{j} \eta_{k} \eta_{l} \geq \lambda_{0}>0
$$

and

$$
\Lambda(x, u, p)=\sup _{|\xi|=|\eta|=1}\left|A_{i j}^{k l}(x, u, p) \xi_{i} \xi_{j} \eta_{k} \eta_{l}\right| \leq \mu_{0} \lambda(x, u, p)
$$

for $x \in \Omega,|u| \leq M$, for positive constants $\lambda_{0}$ and $\mu_{0}$, depending on $M$. Then the estimates (1.24), (3.55) and (3.56) also hold for $A$ satisfying (3.72), (3.73), $\mathcal{F}$ and $B$ satisfying (i) of Theorems 1.3 and 3.1 .

\subsection{Hölder estimates}

In this subsection, we will prove a Hölder estimate for admissible functions $u$ of the augmented Hessian equation (1.1) in the cones $\Gamma_{k}$ for $k>n / 2$, when $A \geq O\left(|p|^{2}\right) I$. For $M[u] \in \Gamma_{k}$ for $n / 2<k \leq n$, we have

$$
F_{k}(M[u])>0, \quad \text { in } \Omega,
$$

where the operator $F_{k}=\left(S_{k}\right)^{\frac{1}{k}}$. Here the condition $A \geq O\left(|p|^{2}\right) I$ is interpreted as the natural quadratic structure condition from below, namely

$$
A(x, z, p) \geq-\mu_{0}\left(1+|p|^{2}\right) I
$$


for all $x \in \Omega,|z| \leq M, p \in \mathbb{R}^{n}$, and some positive constant $\mu_{0}$ depending on the constant $M$. The one-sided quadratic condition (3.75) has already been used for the gradient estimate in the Monge-Ampère case, for the Dirichlet problem in [17] and the Neumann and oblique problems in [16].

Lemma 3.1 Let $u \in C^{2}(\Omega)$ satisfy $M[u] \in \Gamma_{k}$ for $n / 2<k \leq n$ where the matrix $A$ satisfies (3.75). Then for any ball $B_{R}=B_{R}(y)$, with centre $y \in \bar{\Omega}, x \in \Omega_{R}:=B_{R} \cap \Omega$ and $\alpha=2-n / k$, we have the estimate

$$
|u(x)-u(y)| \leq C|x-y|^{\alpha}\left(R^{-\alpha} \underset{\Omega_{R}}{\operatorname{osc}} u+1\right),
$$

provided one of the following holds:

(i) $B_{R} \subset \Omega, u \in C^{0}\left(\bar{\Omega}_{R}\right)$ and the constant $C$ depends on $n, k, \mu_{0}$, osc $u$ and diam $\Omega$;

(ii) $\Omega \in C^{2}$ is convex, $u \in C^{1}\left(\bar{\Omega}_{R}\right)$ and $C$ depends additionally on $\Omega$ and $\inf _{B_{R} \cap \partial \Omega} D_{\nu} u$;

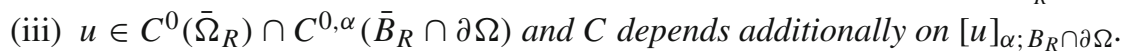

Proof First we consider the interior case (i). For any ball $B_{R}=B_{R}(y) \subset \Omega$, we need to compare the following two functions in $B_{R}$,

$$
v(x)=e^{K(u(x)-u(y))}+\frac{a}{2}|x-y|^{2}-1,
$$

and

$$
\Phi(x)=c|x-y|^{\alpha}, \quad \alpha=2-\frac{n}{k}
$$

where $n / 2<k \leq n, K, a$ and $c$ are positive constants to be determined. By direct calculation, we first observe that

$$
F_{k}\left(D^{2} \Phi\right)=0, \quad \text { for } x \neq y \text {. }
$$

Denoting $\tilde{\Phi}=\Phi+\frac{\epsilon}{2}|x-y|^{2}$ for some positive constant $\epsilon<1$, then the perturbation function $\tilde{\Phi}$ of $\Phi$ satisfies $D^{2} \tilde{\Phi} \in \Gamma_{k}$ in $B_{R}$. Fixing a constant $\rho<\min \{R, 1\}$, it is readily checked that $F_{k}\left(D^{2} \tilde{\Phi}\right)$ is a strictly decreasing function with respect to $|x-y|$ for $x \in B_{R} \backslash B_{\rho}$, and hence

$$
F_{k}\left(D^{2} \tilde{\Phi}\right)<\left.F_{k}\left(D^{2} \tilde{\Phi}\right)\right|_{x \in \partial B_{\rho}} \leq C(n, k, c)\left(\frac{\epsilon}{\rho^{n(k-1) / k}}\right)^{\frac{1}{k}}, \quad \text { for } x \in B_{R} \backslash B_{\rho},
$$

where $B_{\rho}:=B_{\rho}(y), C(n, k, c)$ is a positive constant depending on $n, k$ and $c$. By introducing

$$
S_{k}^{i j}:=S_{k}^{i j}\left(D^{2} \tilde{\Phi}\right)=\frac{\partial S_{k}\left(D^{2} \tilde{\Phi}\right)}{\partial \tilde{\Phi}_{i j}}, \quad \text { and } F_{k}^{i j}:=F_{k}^{i j}\left(D^{2} \tilde{\Phi}\right)=\frac{\partial F_{k}\left(D^{2} \tilde{\Phi}\right)}{\partial \tilde{\Phi}_{i j}}
$$


we have

$$
S_{k}^{i j}=k F_{k}^{k-1}\left(D^{2} \tilde{\Phi}\right) F_{k}^{i j}
$$

We also denote $\mathscr{T}_{S_{k}}=\operatorname{trace}\left(S_{k}^{i j}\right)=(n-k+1) S_{k-1}$. Since $D^{2} \tilde{\Phi} \in \Gamma_{k}$ in $B_{R}$, we have $\left\{F_{k}^{i j}\right\}>0$ and $\left\{S_{k}^{i j}\right\}>0$ in $B_{R}$.

For our desired comparison of the functions $v$ in (3.77) and $\Phi$ in (3.78) on $B_{R}$, we shall first compare them on $B_{R} \backslash B_{\rho}$ for a fixed $\rho$, and then send $\rho$ to 0 in the end. For convenience of later discussion, we now introduce some notation and fix some constants in advance. We denote

$$
\omega_{R}=\underset{B_{R}}{\operatorname{osc}} u, \quad \tilde{K}_{M}=K e^{K \omega_{R}}, \quad \tilde{K}_{m}=K e^{-K \omega_{R}},
$$

and

$$
\delta(n, k)= \begin{cases}1 /(n-k+1), & n / 2<k<n, \\ 1 / n, & k=n .\end{cases}
$$

We can fix the constant $K$ large such that $K>\mu_{0} / \delta(n, k)$, and fix the constant $a$ such that $a>1+\mu_{0} \tilde{K}_{M}$. For fixed $K$, by choosing

$$
c \geq R^{\frac{n}{k}}\left[R^{-2}\left(e^{K \omega_{R}}-1\right)+\frac{a}{2}\right],
$$

we have $v-\Phi \leq 0$ on $\partial B_{R}$. Now the constant $c$ has been fixed as well. For fixed $\rho$ and $c$, by choosing $\epsilon$ sufficiently small such that the quantity on the right hand side of (3.80) is sufficiently small, we can have

$$
F_{k}\left(D^{2} \tilde{\Phi}\right)<\tilde{K}_{m} \inf _{\Omega} F_{k}(M[u]), \quad \text { for } x \in B_{R} \backslash B_{\rho},
$$

where $\tilde{K}_{m}$ is the constant defined in (3.83). If $v-\Phi$ attains its maximum over $\overline{B_{R} \backslash B_{\rho}}$ at a point $x_{0} \in B_{R} \backslash B_{\rho}$, then we have $D(v-\Phi)=0$ and $D^{2}(v-\Phi) \leq 0$ at $x_{0}$, namely

$$
u_{i}\left(x_{0}\right)=\tilde{K}^{-1}\left[-a+\alpha c\left|x_{0}-y\right|^{-\frac{n}{k}}\right]\left[\left(x_{0}\right)_{i}-y_{i}\right], \text { for } i=1, \ldots, n,
$$

and

$$
S_{k}^{i j} D_{i j}(v-\Phi) \leq 0, \quad \text { at } x_{0}
$$

where $\tilde{K}=K e^{K\left(u\left(x_{0}\right)-u(y)\right)}$. Without loss of generality, by rotation of the coordinates, we can assume $x_{0}-y=\left(\left(x_{0}\right)_{1}-y_{1}, 0, \ldots, 0\right)$. From (3.86), we have $D u\left(x_{0}\right)=$ $\left(u_{1}\left(x_{0}\right), 0, \ldots, 0\right)$. By calculation, we have

$$
D^{2} \Phi\left(x_{0}\right)=\alpha c\left|x_{0}-y\right|^{-\frac{n}{k}} \operatorname{diag}\left(1-\frac{n}{k}, 1, \ldots, 1\right)
$$


which has a negative eigenvalue $\Phi_{11}\left(x_{0}\right)$ when $n / 2<k<n$ and has a null eigenvalue $\Phi_{11}\left(x_{0}\right)$ when $k=n$. Correspondingly, for small $\epsilon$, the perturbed Hessian $D^{2} \tilde{\Phi}\left(x_{0}\right)=$ $D^{2} \Phi\left(x_{0}\right)+\epsilon I$ is diagonal, and has a negative eigenvalue $\tilde{\Phi}_{11}\left(x_{0}\right)$ when $n / 2<k<n$ and has a least positive eigenvalue $\tilde{\Phi}_{11}\left(x_{0}\right)$ when $k=n$. Note also that the matrices $\left\{F_{k}^{i j}\right\}$ and $\left\{S_{k}^{i j}\right\}$ are diagonal at $x_{0}$. From the properties of the $k$-Hessian operator and the Monge-Ampère operator, we have

$$
S_{k}^{11} \geq \delta(n, k) \mathscr{T}_{S_{k}}
$$

where $\delta(n, k)$ is defined in (3.84).

By our choices of the constants $K$ and $a$, from $w_{i j}=u_{i j}-A_{i j}$, (3.75), (3.85), (3.82), (3.89), and the concavity and homogeneity of $F_{k}$, we have, at $x_{0}$,

$$
\begin{aligned}
S_{k}^{i j} D_{i j}(v-\Phi)= & S_{k}^{i j}\left[K \tilde{K} u_{i} u_{j}+\tilde{K} A_{i j}+(a-\epsilon) \delta_{i j}+\tilde{K} w_{i j}-\tilde{\Phi}_{i j}\right] \\
\geq & K \tilde{K} S_{k}^{11} u_{1}^{2}+S_{k}^{i j}\left[-\mu_{0} \tilde{K}\left(1+|D u|^{2}\right) \delta_{i j}+(a-\epsilon) \delta_{i j}\right] \\
& +k F_{k}^{k-1}\left(D^{2} \tilde{\Phi}\right) F_{k}^{i j}\left[\tilde{K} w_{i j}-\tilde{\Phi}_{i j}\right] \\
\geq & \tilde{K}_{m}\left(K \delta(k, n)-\mu_{0}\right) \mathscr{T}_{S_{k}}|D u|^{2}+\left(a-\epsilon-\mu_{0} \tilde{K}_{M}\right) \mathscr{T}_{S_{k}} \\
& +k F_{k}^{k-1}\left(D^{2} \tilde{\Phi}\right)\left[\tilde{K}_{m} F_{k}(M[u])-F_{k}\left(D^{2} \tilde{\Phi}\right)\right] \\
> & 0 .
\end{aligned}
$$

The contradiction from (3.87) and (3.90) shows that $v-\Phi$ must take its maximum over $\overline{B_{R} \backslash B_{\rho}}$ at the boundary $\partial B_{R}$ or $\partial B_{\rho}$. Therefore, we have

$$
v-\Phi \leq \max \left\{0, \sup _{B_{\rho}}(v-\Phi)\right\}, \quad \text { for } x \in \overline{B_{R} \backslash B_{\rho}}
$$

We observe that we can choose $\epsilon$ as small as we want in (3.90). Letting $\epsilon \rightarrow 0$ in (3.90), the inequality (3.91) can hold for $\rho$ as small as we want. Sending $\rho \rightarrow 0$ and using the forms of $v$ in (3.77) and $\Phi$ in (3.78), the right hand side of (3.91) tends to 0 . Correspondingly, we have from (3.91),

$$
v \leq \Phi, \quad \text { for } x \in \bar{B}_{R}
$$

namely

$$
u(x)-u(y) \leq \frac{1}{K} \log \left(1+c|x-y|^{\alpha}\right), \quad \text { for } x \in \bar{B}_{R},
$$

and hence assertion (i) follows by controlling $c$ from above, for example by estimating, $c \leq R^{-\alpha} \omega_{R} \tilde{K}_{M}+\frac{a}{\alpha}$.

Remark 3.8 In the above argument, we use the perturbation $\tilde{\Phi}=c|x-y|^{\alpha}+\frac{\epsilon}{2}|x-y|^{2}$ of the function $\Phi=c|x-y|^{\alpha}$. We remark that there are alternative perturbations that can be used here. For instance, we can choose a perturbation in the form, $\tilde{\Phi}=$ $c\left(|x-y|^{2}+\epsilon^{2}\right)^{\frac{\alpha}{2}}$ for small $\epsilon$. 
If we consider admissible functions $u$ of equation (1.1) in the cones $\Gamma_{k}$ for $k>n / 2$ satisfying various boundary conditions, we can also have similar comparison in (3.93) locally near the boundary. For the Neumann case (ii), we suppose $B_{R}$ intersects $\partial \Omega$, with $\Omega_{R}$ convex and $D_{\nu} u>-\kappa$ on $\partial \Omega \cap B_{R}$, where $\kappa$ is a nonnegative constant. Defining $\tilde{u}=u-\kappa \phi$, where $\phi$ is a negative defining function of $\Omega$, as in Sects. 3.1 and 3.2, satisfying $\phi<0$ in $\Omega, \phi=0$ on $\partial \Omega$ and $D_{\nu} \phi=-1$ on $\partial \Omega$, then we have $D_{\nu} \tilde{u}>0$ on $\partial \Omega \cap B_{R}$. Using $\tilde{u}$ in place of $u$ in (3.77), we then compare the replaced function $v$ in (3.77) and the function $\Phi$ in (3.78) on $\bar{\Omega}_{R}$. Similar to the interior case, we begin with our discussion on $\overline{\left(B_{R} \backslash B_{\rho}\right) \cap \Omega}$ for a fixed $\rho<\min \{R, 1\}$, where $B_{\rho}:=B_{\rho}(y)$. If the maximum of $v-\Phi$ takes its maximum over $\overline{\left(B_{R} \backslash B_{\rho}\right) \cap \Omega}$ at a point $x_{0} \in\left(B_{R} \backslash B_{\rho}\right) \cap \Omega$, by choosing a larger constant $a$ depending additionally on $\kappa,|\phi|_{2 ; \Omega}$, we can obtain the same inequality as in (3.90) and get a contradiction with (3.87). Therefore, the possibilities that maximum point $x_{0}$ of $v-\Phi$ occurs are on $\left(B_{R} \backslash B_{\rho}\right) \cap \partial \Omega, \partial B_{R} \cap \Omega$ or $\partial B_{\rho} \cap \Omega$. If $x_{0} \in\left(B_{R} \backslash B_{\rho}\right) \cap \partial \Omega$, from the convexity of $\Omega_{R}$, we have $\left(x_{0}-y\right) \cdot v\left(x_{0}\right) \leq 0$. Then we have, at $x_{0}$,

$$
\begin{aligned}
0 & \geq D_{v}(v-\Phi)\left(x_{0}\right) \\
& =K e^{K\left(\tilde{u}\left(x_{0}\right)-\tilde{u}(y)\right)} D_{\nu} \tilde{u}\left(x_{0}\right)+\left[a-\alpha c\left|x_{0}-y\right|^{-\frac{n}{k}}\right]\left(x_{0}-y\right) \cdot v\left(x_{0}\right) \\
& >0
\end{aligned}
$$

by using $D_{\nu} \tilde{u}>0$ on $\partial \Omega$, and choosing the constant $c$ large such that $c \geq a R^{\frac{n}{k}} / \alpha$. Then (3.94) leads to a contradiction and excludes the case that the maximum of $v-\Phi$ occurs at $\left(B_{R} \backslash B_{\rho}\right) \cap \partial \Omega$. By fixing the defining function $\phi$ such that $\phi>-1 / \kappa$, we have $\kappa(\phi(x)-\phi(y))>-1$ for $x \in \partial B_{R} \cap \Omega$ and $y \in \partial \Omega$. With this property of the defining function, now by choosing $c$ larger again such that

$$
c \geq R^{\frac{n}{k}}\left[R^{-2}\left(e^{K\left(\omega_{R}+\kappa \operatorname{osc} \phi\right)}-1\right)+\frac{a}{2}\right]
$$

we have $v-\Phi \leq 0$ on $\partial B_{R} \cap \Omega$. Similarly to (3.91) of the interior case, with $\mu_{0}$ and $K$ appropriately adjusted, we now have

$$
v-\Phi \leq \max \left\{0, \sup _{B_{\rho} \cap \Omega}(v-\Phi)\right\}, \quad \text { for } x \in \overline{\left(B_{R} \backslash B_{\rho}\right) \cap \Omega} .
$$

Therefore, by successively passing $\epsilon$ and $\rho$ to 0 , the same inequality (3.93) holds on $\bar{\Omega}_{R}$ and hence assertion (ii) is proved.

Finally for Dirichlet boundary values, as in case (iii), we suppose again that $B_{R}$ intersects $\partial \Omega$ and $u \in C^{0}\left(\bar{\Omega}_{R}\right) \cap C^{0, \alpha}\left(\bar{B}_{R} \cap \partial \Omega\right)$ so that

$$
|u(x)-u(y)| \leq \kappa|x-y|^{\alpha},
$$

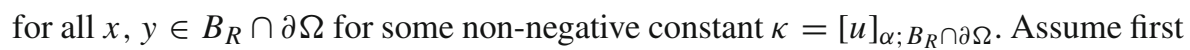
that the centre $y \in \partial \Omega$. Then proceeding as in the previous case we need to compare $v$ and $\Phi$ on $\left(B_{R} \backslash B_{\rho}\right) \cap \partial \Omega$. Accordingly we now obtain 


$$
\begin{aligned}
v-\Phi & =e^{K(u(x)-u(y))}-1+\frac{a}{2}|x-y|^{2}-c|x-y|^{\alpha} \\
& \leq \kappa K e^{K \omega_{R}^{\prime}}|x-y|^{\alpha}+\frac{a}{2}|x-y|^{2}-c|x-y|^{\alpha} \\
& \leq 0, \quad \text { for } x \in\left(B_{R} \backslash B_{\rho}\right) \cap \partial \Omega,
\end{aligned}
$$

by taking $c$ larger such that

$$
c \geq \kappa K e^{K \omega_{R}^{\prime}}+\frac{a}{2} R^{2-\alpha},
$$

where $\omega_{R}^{\prime}=\underset{\partial \Omega \cap B_{R}}{\operatorname{osc}} u$. With $K$ and $c$ also chosen as in case (i), with $B_{R}$ replaced by $\Omega_{R}$, we arrive again at (3.93) on $\bar{\Omega}_{R}$.

The general case, $y \in \Omega$, in case (iii), now follows by combining the case, $y \in \partial \Omega$ with the interior estimate, (3.76) in case (i), as in Theorem 8.29 in [7].

For convex domains, Lemma 3.1 extends the gradient bound, Lemma 3.2 in [16], for the case $k=n$. More generally it provides a modulus of continuity estimate for solutions of (1.1) that are admissible in $\Gamma_{k}$ for $k>n / 2$. Combining with the local gradient estimate in Theorem 3.1, the estimate (3.55) can hold by extending " $o$ " to " $O$ " in (1.22) and (3.33). For a convex domain $\Omega$, the estimate (3.56) can still hold for the semilinear Neumann problems in case (ii) of Theorem 3.1 by extending " $o$ " to " $O$ " in (1.22) and (3.33).

\section{Existence and applications}

In this section, we present some existence results for classical solutions based on our first and second derivative a priori estimates for admissible solutions for the oblique boundary value problem (1.1)-(1.2). We also give various examples of equations and boundary conditions satisfying our conditions and also show that our theory can be extended to embrace $C^{1,1}$ solutions of degenerate equations.

\subsection{Existence theorems}

We assume that $\underline{u}$ and $\bar{u}$ in $C^{2}(\Omega) \cap C^{1}(\bar{\Omega})$ are respectively an admissible subsolution and supersolution of the boundary value problem (1.1)-(1.2), satisfying the inequalities (1.25) and (1.26), with $\mathcal{F}$ satisfying $\mathrm{F} 1$ and $\mathcal{G}$ oblique. Under the assumptions $A$, $B$ and $G$ are non-decreasing in $z$, with at least one of them strictly increasing, if $u \in C^{2}(\Omega) \cap C^{1}(\bar{\Omega})$ is an admissible solution of the problem (1.1)-(1.2), by the comparison principle, we have

$$
\underline{u} \leq u \leq \bar{u}, \quad \text { in } \bar{\Omega} .
$$

For our purposes here we note that the comparison principle, as formulated in Lemma 3.1 in [16], extends automatically to operators $\mathcal{F}$ satisfying F1. Then (4.1) provides the solution bound and the interval $\mathcal{I}=[\underline{u}, \bar{u}]$ for the convexity definition (1.16). 
With the a priori estimates up to second order, we can formulate existence results for the classical admissible solutions of the oblique boundary value problems (1.1)(1.2). We consider first the case when the matrix $A$ is strictly regular and the boundary operator $\mathcal{G}$ is semilinear.

Theorem 4.1 Assume that $F$ satisfies conditions F1-F4 in the cone $\Gamma \subset \mathcal{P}_{n-1}, \Omega$ is a bounded $C^{3,1}$ domain in $\mathbb{R}^{n}, A \in C^{2}\left(\bar{\Omega} \times \mathbb{R} \times \mathbb{R}^{n}\right)$ is strictly regular in $\bar{\Omega}$, $B>a_{0}, \in C^{2}\left(\bar{\Omega} \times \mathbb{R} \times \mathbb{R}^{n}\right), \mathcal{G}$ is semilinear and oblique, with $\beta \in C^{2,1}(\partial \Omega)$, $\varphi \in C^{2,1}(\partial \Omega \times \mathbb{R}), \underline{u}$ and $\bar{u}, \in C^{2}(\Omega) \cap C^{1}(\bar{\Omega})$ are respectively an admissible subsolution and a supersolution of the oblique boundary value problem (1.1)-(1.2) with $\Omega$ uniformly $(\Gamma, A, G)$-convex with respect to the interval $\mathcal{I}=[\underline{u}, \bar{u}]$. Assume also that $A, B$ and $\varphi$ are non-decreasing in $z$, with at least one of them strictly increasing, and that $A$ and $B$ satisfy the quadratic growth conditions (1.22), with $B \geq O$ (1) if $a_{0}=-\infty$. Assume either F5 $5^{+}$holds or $B$ is independent of $p$. Then if one of the following further conditions is satisfied:

(i) A is uniformly regular, $\mathcal{F}$ satisfies $F 5$, with $b=\infty$ and $B-p \cdot D_{p} B \leq o\left(|p|^{2}\right)$ in (1.22);

(ii) $\beta=v, \mathcal{F}$ is orthogonally invariant and satisfies $F 7$ and either (a) $A=o\left(|p|^{2}\right)$ in (1.22) or (b) $\Gamma \subset \Gamma_{k}$ with $k>n / 2$ and $\Omega$ is convex,

(iii) $\Gamma=\Gamma_{n}$ and A satisfies (3.75) in place of (1.22),

there exists a unique admissible solution $u \in C^{3, \alpha}(\bar{\Omega})$ of the boundary value problem (1.1)-(1.2), for any $\alpha<1$.

Proof First we note that the uniqueness follows directly from the comparison principle, using our monotonicity condtions on $A, B$ and $G$. To prove the existence assertion, under the assumptions of Theorem 4.1, we have solution bounds (4.1), gradient estimates (1.24) from Sect. 3 and second derivative estimates (1.18) by Theorem 1.2. Note that the gradient estimate when $\Gamma=\Gamma_{k}$ with $k>n / 2$ and $\Omega$ is convex in case (ii) is obtained by combining the local gradient estimate in Theorem 3.1 and the Hölder estimate in Lemma 3.1; (see the last paragraph of Sect. 3). From the uniformly elliptic theory $[25,26,35]$, we can then derive a global second derivative Hölder estimate

$$
|u|_{2, \alpha ; \Omega} \leq C
$$

for admissible solutions $u \in C^{4}(\Omega) \cap C^{3}(\bar{\Omega})$ of the semilinear oblique boundary value problem (1.1)-(1.2) for $\alpha \in(0,1)$. With the $C^{2, \alpha}$ estimate, by choosing the subsolution $\underline{u}$ as an initial solution, we can employ the classical method of continuity, Theorem 17.22 and Theorem 17.28, in [7] to derive the existence of an admissible solution $u \in C^{2, \alpha}(\bar{\Omega})$. In order to preserve our subsolution and supersolution inequalities and guarantee the uniform a priori estimates, we initially assume that $A, B \in C^{2, \gamma}(\bar{\Omega} \times$ $\left.\mathbb{R} \times \mathbb{R}^{n}\right)$ and $\underline{u} \in C^{4, \gamma}(\bar{\Omega})$ for some $\gamma \in(0,1)$ and consider the family of problems:

$$
\begin{aligned}
& \mathcal{F}[u]=B(\cdot, u, D u)+(1-\sigma)\{\mathcal{F}[\underline{u}]-B(\cdot, \underline{u}, D \underline{u})\}, \quad \text { in } \Omega, \\
& \mathcal{G}[u]=(1-\sigma) \mathcal{G}[\underline{u}], \quad \text { on } \partial \Omega,
\end{aligned}
$$

for $0 \leq \sigma \leq 1$. Then any admissible solution $u \in C^{2, \alpha}(\bar{\Omega})$ of (4.3) satisfies $u \in$ $C^{4, \gamma}(\bar{\Omega}) \cap C^{3, \gamma}(\bar{\Omega})$ and the estimates (4.1), (4.2) uniformly in $\sigma$. Note that for this we 
only need the boundary data $\partial \Omega \in C^{3, \gamma}, \beta, \varphi \in C^{2, \gamma}$. Since $\underline{u}$ is the unique solution of (4.3) at $\sigma=0$, we then obtain the existence of a unique solution $u \in C^{4}(\Omega) \cap C^{3}(\bar{\Omega})$ at $\sigma=1$.

To prove Theorem 4.1 as stated, we then need to suitably approximate $A, B$ and $\underline{u}$. Accordingly we let $A^{(m)}, B^{(m)} \in C^{2, \gamma}\left(\bar{\Omega} \times \mathbb{R} \times \mathbb{R}^{n}\right), m=1,2, \ldots$, satisfy $D^{\alpha} A^{(m)} \rightarrow$ $D^{\alpha} A, D^{\alpha} B^{(m)} \rightarrow D^{\alpha} B$, locally uniformly in $\bar{\Omega} \times \mathbb{R} \times \mathbb{R}^{n}$ for any multi-index $|\alpha| \leq 2$. To approximate $\underline{u}$, we assume initially $\underline{u}, \bar{u} \in C^{2}(\bar{\Omega})$ and let $\underline{u}^{(m)} \in C^{4, \gamma}(\bar{\Omega})$ satisfy $D^{\alpha} \underline{u}^{(m)} \rightarrow D^{\alpha} \underline{u}$ uniformly in $\Omega$ for all $|\alpha| \leq 2$, together with $\underline{u}^{(m)} \leq \underline{u}$ and $D \underline{u}^{(m)} \geq$ $D_{\beta} \underline{u}$ on $\partial \Omega$ for all $m=1,2, \ldots$. We now consider the approximating boundary value problems:

$\mathcal{F}^{(m)}[u]:=F\left[D^{2} u-A^{(m)}(\cdot, u, D u)\right]=B^{(m)}(\cdot, u, D u)$ in $\Omega, \quad \mathcal{G}[u]=0$ on $\partial \Omega$.

From the definition of $(\Gamma, A, G)$-convexity, we see that $\Omega$ will be uniformly $(\Gamma, A, G)$ convex with respect to an interval $\mathcal{I}_{\delta}=[\underline{u}-\delta, \bar{u}+\delta]$ for some $\delta>0$. Assuming first that either $A$ or $B$ is strictly increasing with respect to $z$, we then have, (using condition F5 in the first case), that $\underline{u}-\delta$ and $\bar{u}+\delta$ are respectively an admissible subsolution and a supersolution of the oblique boundary value problem (4.4) for $m$ sufficiently large and we thus obtain the estimate (4.2) for admissible solutions $u \in C^{4}(\Omega) \cap C^{3}(\bar{\Omega})$ of (4.4) for $m$ sufficiently large, with constants $\alpha$ and $C$ independent of $m$. Furthermore it also follows that $u^{(m)}-\delta \in C^{4, \gamma}$ is an admissible subsolution of (4.4) for $m$ sufficiently large so we also obtain the existence of a unique admissible solution $u^{(m)} \in C^{4, \gamma}(\Omega) \cap C^{3, \gamma}(\bar{\Omega})$ of (4.4) satisfying, by virtue of the linear Schauder theory [7, Chapter 6] and (4.2), the estimates

$$
\left|u^{(m)}\right|_{3, \alpha ; \Omega} \leq C,
$$

for any $\alpha<1$, and constant $C$ independent of $m$. Letting $m \rightarrow \infty$, we then establish Theorem 4.1 in the cases where $A$ or $B$ are strictly increasing, and $\underline{u} \in C^{2}(\bar{\Omega})$.

For the remaining case when $\varphi$ is strictly increasing with respect to $z$, or equivalently $G$ is strictly decreasing with respect to $z$, we use the uniform ellipticity of $\mathcal{F}$ with respect to $\underline{u}, \bar{u}$ and the strict monotonicity of $G$ to construct admissible subsolutions $\underline{v}$ and supersolutions $\bar{v}$ of (4.4) in the form

$$
\underline{v}=\underline{u}-\delta+\delta^{\prime} e^{K x_{1}}, \quad \bar{v}=\bar{u}+\delta-\delta^{\prime} e^{K x_{1}},
$$

for sufficiently large constant $K$, small $\delta^{\prime}$ satisfying $0<\delta^{\prime} e^{K x_{1}}<\delta / 2$ and sufficiently large $m$. Clearly $v^{(m)}=\underline{u}^{(m)}-\delta+\delta^{\prime} e^{K x_{1}} \in C^{4, \gamma}(\bar{\Omega})$ is also an admissible subsolution for sufficiently large $m$ so we are able to establish Theorem 4.1 also in this case. Finally to remove our conditions $\underline{u}, \bar{u} \in C^{2}(\bar{\Omega})$, we may replace $\Omega$ by a parallel approximating domain $\Omega_{\epsilon}=\{x \in \Omega \mid \operatorname{dist}(x, \partial \Omega)>\epsilon\}$ for $\epsilon>0$ sufficiently small, with $G$ extended to $\partial \Omega_{\epsilon} \times \mathbb{R} \times \mathbb{R}^{n}$ to be constant along normals to $\partial \Omega$. Letting $\epsilon \rightarrow 0$, we then obtain Theorem 4.1 it its full generality. 
With the above existence result for general operators, the existence for semilinear oblique problem (1.1)-(1.2) of the $k$-Hessian and Hessian quotient equations, $\mathcal{F}=F_{k, l}$ for $0 \leq l<k \leq n, k>1$ in Theorem 1.4, is just a special case. The conditions in cases (i), (ii) in Theorem 1.4 agree with those in (i), (ii) in Theorem 4.1, respectively. For case (iii) of Theorem 1.4, the gradient estimate follows from Lemma 3.2 in [16], while second derivative estimate is from Theorem 1.2. In the special case when $k=1$, equation (1.1) reduces to a quasilinear Poisson equation, as the matrix $A$ can then be absorbed in the scalar $B$ and considerably more general results for arbitrary smooth domains $\Omega$ follow from the classical Schauder theory [7]. In particular we need only assume the quadratic growth, $B=O\left(|p|^{2}\right)$ as $|p| \rightarrow \infty$, uniformly for $x \in \Omega,|z| \leq$ $M$ for any $M>0$, and under reduced smoothness hypotheses, $B \in C^{0, \alpha}\left(\bar{\Omega} \times \mathbb{R} \times \mathbb{R}^{n}\right)$, $\partial \Omega \in C^{1, \alpha}, \beta \in C^{1, \alpha}(\partial \Omega), \varphi \in C^{1, \alpha}(\partial \Omega \times \mathbb{R})$, we infer the existence of a unique classical solution $u \in C^{2, \alpha}(\bar{\Omega})$ of the semilinear oblique problem (1.1)-(1.2).

Using Lemma 4.1 in [16] and the nonlinear case in Theorem 1.2, we can extend Theorem 4.1 to cover nonlinear boundary operators in the case where $\Gamma$ is the positive cone $\Gamma_{n}$. For this we also need to assume that $\mathcal{G}$ is uniformly oblique in the sense that

$$
G_{p}(x, z, p) \cdot v \geq \beta_{0}, \quad\left|G_{p}(x, z, p)\right| \leq \sigma_{0}, \quad \text { on } \partial \Omega,
$$

for all $x \in \Omega,|z| \leq M, p \in \mathbb{R}^{n}$ and positive constants $\beta_{0}$ and $\sigma_{0}$, depending on the constant $M$. The following existence result, which is proved similarly to Theorem 4.1, extends the Monge-Ampère case, Theorem 4.2 in [16] as well as case (iii) in Theorem 1.4.

Theorem 4.2 Assume that $F$ satisfies conditions F1-F4 and F6 in the positive cone $\Gamma_{n}, \Omega$ is a bounded $C^{3,1}$ domain in $\mathbb{R}^{n}, A \in C^{2}\left(\bar{\Omega} \times \mathbb{R} \times \mathbb{R}^{n}\right)$ is strictly regular in $\bar{\Omega}$, $B>a_{0}, \in C^{2}\left(\bar{\Omega} \times \mathbb{R} \times \mathbb{R}^{n}\right), G \in C^{2,1}\left(\partial \Omega \times \mathbb{R} \times \mathbb{R}^{n}\right)$ is concave with respect to $p$ and uniformly oblique in the sense of (4.5), $\underline{u}$ and $\bar{u}, \in C^{2}(\Omega) \cap C^{1}(\bar{\Omega})$ are respectively an admissible subsolution and a supersolution of the oblique boundary value problem (1.1)-(1.2) with $\Omega$ uniformly $(A, G)$-convex with respect to the interval $\mathcal{I}=[\underline{u}, \bar{u}]$. Assume also that $A, B$ and $-G$ are non-decreasing in $z$, with at least one of them strictly increasing and A satisfies the quadratic growth condition (3.75). Assume either $\mathrm{F5}^{+}$holds or $B$ is independent of $p$. Then there exists a unique admissible solution $u \in C^{3, \alpha}(\bar{\Omega})$ of the boundary value problem (1.1)-(1.2), for any $\alpha<1$.

Remark 4.1 Without the monotonicity, subsolution and supersolution hypotheses in Theorems 4.1 and 4.2 we can still obtain the existence of possibly non-unique, admissible solutions of the oblique boundary value problem (1.1)-(1.2), using topological fixed point theorems, (cf. Theorem 11.6 in [7]), or degree theory, (as in [47]), instead of the method of continuity, provided we have a priori bounds for solutions of appropriate families such as (4.3), so that their ranges lie in fixed intervals $\mathcal{I}$, where $\partial \Omega$ is uniformly $(\Gamma, A, G)$-convex. Note also that the monotonicity conditions themselves may be relaxed somewhat to get the inequality (4.1). In particular we can strengthen the sub and super solution properties of $\underline{u}$ and $\bar{u}$ so that $D^{2} \underline{u} \geq A(x, z, D \underline{u}(x))$ and

$$
\begin{aligned}
F\left[D^{2} \underline{u}-A(x, z, D \underline{u}(x))\right] & \geq B(x, z, D \underline{u}(x)), & & \text { in } \Omega, \\
G(x, z, D \underline{u}(x)) & \geq 0, & & \text { on } \partial \Omega,
\end{aligned}
$$


whenever $z<\underline{u}(x)$, with one of the inequalities in (4.6) strict, and

$$
\begin{aligned}
F\left[D^{2} \bar{u}-A(x, z, D \bar{u}(x))\right] & \leq B(x, z, D \bar{u}(x)), & & \text { in } \Omega, \\
G(x, z, D \bar{u}(x)) & \leq 0, & & \text { on } \partial \Omega,
\end{aligned}
$$

whenever $z>\bar{u}(x), D^{2} \bar{u} \geq A(x, z, D \bar{u}(x))$, with one of (4.7) strict. As a special case, if $\underline{u}=-K, \bar{u}=K$ for some constant $K$, we get $|u| \leq K$; (see also [16], Sect. 3). Under this more general hypothesis we can then infer the existence of admissible solutions in Theorems 4.1 and 4.2.

Remark 4.2 By further approximating $G$ and $\Omega$ in the proof of Theorems 4.1 and 4.2, we need only assume $G \in C^{2}\left(\partial \Omega \times \mathbb{R} \times \mathbb{R}^{n}\right)$ and $\partial \Omega \in C^{3}$, (in accordance with the smoothness hypotheses of Theorems 1.2 and 1.3), to obtain admissible solutions $u \in C^{3, \alpha}(\Omega) \cap C^{2, \alpha}(\bar{\Omega})$, for all $\alpha<1$.

We can also avoid approximating $A$ and $B$ in the proof of Theorems 4.1 and 4.2 by using the linear $L^{p}$ regularity theory, ([7], Chapter 9), which implies, under our smoothness hypotheses on $A$ and $B$, that a $C^{2, \alpha}$ solution of (1.1)-(1.2) lies in the Sobolev space $W_{\text {loc }}^{4, p}(\Omega)$, for any $p<\infty$. Then we may carry out the derivation of the estimate (4.2) by applying the Aleksandrov-Bakel'man maximum principle to our differential inequalities, rather than the classical comparison arguments. Moreover through this approach or by further refinement of our approximation arguments, we need only assume $A, B \in C^{1,1}\left(\bar{\Omega} \times \mathbb{R} \times \mathbb{R}^{n}\right)$, (with our convexity conditions on $A$ holding a.e.), $G \in C^{1,1}\left(\partial \Omega \times \mathbb{R} \times \mathbb{R}^{n}\right)$ and $\partial \Omega \in C^{2,1}$ to obtain the estimate (4.2) and thereby the existence of a unique admissible solution $u \in C^{3, \alpha}(\Omega) \cap C^{2, \alpha}(\bar{\Omega})$, for all $\alpha<1$.

\subsection{Examples}

In this subsection, we present various examples of operators $\mathcal{F}$, matrix functions $A$ and associated oblique boundary operators $\mathcal{G}$ which satisfy our hypotheses.

Examples for $\mathcal{F}$. As already indicated in Sect. 1, our main examples are the $k$ Hessian operators and their quotients $F_{k, l}(0 \leq l<k \leq n)$, as considered in Theorem 1.4. For $0 \leq l<k \leq n, F_{k, l}$ satisfy F1-F5, F7 in $\Gamma_{k}$ with $a_{0}=0$. We remark that $b$ in F5 can be a positive constant or $+\infty$. For $l=0$, the corresponding $k$-Hessian operators $F_{k}, 2 \leq k \leq n$ satisfy F1-F4, F5 ${ }^{+}$and F7 in $\Gamma_{k}$ with $a_{0}=0$. In this case the operators $F_{k}$ only satisfy $\mathrm{F}^{+}$for finite $b$ but not infinite $b$. Note that the normalised Monge-Ampère operator in the form (det $)^{\frac{1}{n}}$ is also covered by $F_{k}$ when $k=n$. Another well known concave form of the Monge-Ampère operator is $\log (\mathrm{det})$, which satisfies $\mathrm{F} 1-\mathrm{F} 4, \mathrm{~F}^{+}$in $K^{+}$with $a_{0}=-\infty$. As stated in the introduction, the $k$-Hessian operators $F_{k}(k=1, \ldots, n)$ and the Hessian quotients $F_{n, l}(1 \leq l \leq n-1)$ satisfy F6 in the positive cone $K^{+}$. More generally, if $\mathcal{F}$ is an operator satisfying $\mathrm{F} 1$, $\mathrm{F} 2, \mathrm{~F} 3, \mathrm{~F}^{+}$, with finite $a_{0}$, then $\mathrm{F} 6$ holds in $K^{+}$, since

$$
\begin{aligned}
\mathcal{E}_{2} & \leq r \cdot F_{r}|r| \\
& \leq\left(F(r)-a_{0}\right)|r|
\end{aligned}
$$




$$
\begin{aligned}
& \leq\left(b-a_{0}\right)|r| \\
& \leq o(|r|) \mathscr{T}, \quad \text { as }|r| \rightarrow \infty,
\end{aligned}
$$

for $-\infty<a_{0}<F(r) \leq b<\infty$, where the property of $K^{+}$is used in the first inequality, (1.10) is used in the second inequality, and $\mathrm{F}^{+}$is used in the last two lines. Such a property was observed by Urbas $[47,50]$ for orthogonally invariant $\mathcal{F}$. Note that (4.8) here holds for non-orthogonally invariant $\mathcal{F}$ as well.

Also as mentioned in the introduction, when $a_{0}$ is finite, both conditions F4 and F5, (with $a=a_{0}$ ), follow from F1, F2 and F3. To show F5, (independently of F4), we may simply estimate, using F2 and (1.10), for $r_{0} \in \Gamma$ and $F(r)<b$,

$$
\begin{aligned}
\mathscr{T}(r) & \geq r_{0} \cdot F_{r} /\left|r_{0}\right| \\
& \geq\left(F\left(r_{0}\right)-F(r)+r \cdot F_{r}\right) /\left|r_{0}\right| \\
& \geq\left(F\left(r_{0}\right)-b\right) /\left|r_{0}\right| \geq \delta_{0},
\end{aligned}
$$

for some constant $\delta_{0}$, depending on $F$ and $b$, if $F\left(r_{0}\right)$ is sufficiently large. To show F4, we first note from (1.10) that $F(t r)$ is nondecreasing in $t$ for $t>0$ so if the point $r$ lies on the ray from the vertex 0 through $r_{0} \in \Gamma$, we have $F(t r) \geq F\left(r_{0}\right)$ for all $t \geq\left|r_{0}\right| /|r|$. Otherwise we consider the plane spanned by the straight lines joining $r$ and $r_{0} \in \Gamma$ to the vertex of $\Gamma$. Letting $a_{0}=0$ without loss of generality, and using F2, it then follows that for given $\epsilon \in(0,1)$, there exists some $t>0$, such that $F(t r)>(1-\epsilon) F\left(r_{0}\right)$ and hence $\mathrm{F} 4$ follows by choosing $r_{0}$ so that $F\left(r_{0}\right)$ is sufficiently large. Note also that for these arguments, we only need $F$ nondecreasing in $\Gamma$.

Instead of the elementary symmetric functions $S_{k}$, we may also consider functions $P_{k}$, which are products of $k$ sums of eigenvalues, namely

$$
P_{k}[r]:=P_{k}(\lambda(r))=\prod_{i_{1}<\cdots<i_{k}} \sum_{s=1}^{k} \lambda_{i_{s}}(r), \quad k=1, \ldots, n,
$$

defined in the cones

$$
\mathcal{P}_{k}=\left\{r \in \mathbb{S}^{n} \mid \sum_{s=1}^{k} \lambda_{i_{s}}(r)>0\right\},
$$

where $i_{1}, \ldots, i_{k} \subset\{1, \ldots, n\}, \lambda(r)=\left(\lambda_{1}(r), \ldots, \lambda_{n}(r)\right)$ denote the eigenvalues of the matrix $r \in \mathcal{P}_{k}$. In differential geometry, there is a large amount of literature dealing with $k$-convex hypersurfaces, where the notion $k$-convexity of a hypersurface, originating from $[33,34]$, is that the sum of any $k$-principal curvatures at each point is positive. Clearly the associated operators in (4.10) interpolate between the Laplacian, $k=n$, and the Monge-Ampère operator, $k=1$. We then obtain another group of examples satisfying the hypotheses of Theorem 1.2, namely the normalised functions

$$
\tilde{F}_{k}:=\left(P_{k}\right)^{\frac{1}{C_{n}^{k}}}, \quad C_{n}^{k}=\frac{n !}{k !(n-k) !}, \quad 1 \leq k \leq n,
$$


which are homogeneous of degree one and satisfy F1-F5 in $\mathcal{P}_{k}$ with $a_{0}=0$. Note that the associated operators also interpolate between the Laplacian $\tilde{\mathcal{F}}_{n}=\mathcal{F}_{1}$ and the normalised Monge-Ampère operator $\tilde{\mathcal{F}}_{1}=\mathcal{F}_{n}$ and that the concavity $\mathrm{F} 2$ follows from the arithmetic-geometric mean inequality, similarly to the Monge-Ampère case, $k=1$, (or as a consequence by virtue of the general property that concave functions of linear functions are also concave). For $1 \leq k \leq n-1$, the functions $\tilde{F}_{k}$ also satisfy $\mathrm{v}$ in $\mathcal{P}_{k}$ and F6 in the positive cone $K^{+}$. Using the property that at most $k-1$ of $\lambda_{1}(r), \ldots, \lambda_{n}(r)$ can be negative, we also see that $\tilde{F}_{k}$ satisfies F7 in $\mathcal{P}_{k}$. Furthermore from Theorems 1.2 and 1.3, it follows that we can substitute $\tilde{F}_{k}$ for $F_{k}$ and $\mathcal{P}_{k}$ for $\Gamma_{k}$ in cases (i) and (ii)(a) of Theorem 1.4. In the next subsection we will also introduce degenerate versions of these operators.

We also have further examples originating from geometric applications, given by functions,

$$
F_{k,-\alpha}[r]:=F_{k,-\alpha}(\lambda(r))=\left\{\sum_{i_{1}<\cdots<i_{k}}\left[\sum_{s=1}^{k} \lambda_{i_{s}}(r)\right]^{-\alpha}\right\}^{-\frac{1}{\alpha}}, \quad \alpha>0
$$

also defined in the cone $\mathcal{P}_{k}$ for $k=1, \ldots, n$. When $\alpha=k=1, F_{k,-\alpha}$ coincides with the Hessian quotient $F_{n, n-1}$ and if $\kappa=\left(\kappa_{1}, \ldots, \kappa_{n}\right)$ denotes the principal curvatures of a hypersurface in $\mathbb{R}^{n+1}$, then $F_{1,-1}[\kappa]$ is its harmonic curvature while $F_{1,-2}[\kappa]$ is the inverse of the length of the second fundamental form; see [6]. The associated operators are homogeneous and satisfy F1-F5 and F7 in $\mathcal{P}_{k}$ with $a_{0}=0$ and either finite or infinite $b$ in F5.

The operators in the above examples are all orthogonally invariant. We also have examples of operators $\mathcal{F}$ which are not orthogonally invariant. For instance, let us consider a set $V=\left\{Q_{1}, \ldots, Q_{m}\right\}$, where $Q_{i}, i=1, \ldots, m$ are nonsingular matrices and $m>1$ is a finite integer. We can define an operator of the form

$$
F_{k, V}[r]=\min _{Q \in V} F_{k}\left(Q r Q^{-1}\right), \quad \text { for } k=1, \ldots, n,
$$

in the cone $\Gamma_{k, V}=\left\{r \in \mathbb{S}^{n} \mid F_{j}\left(Q r Q^{-1}\right)>0, \forall Q \in V, j=1, \ldots, k\right\}$. Then the operator in (4.14) provides an example, which is non-orthogonally invariant, but still satisfies our assumptions $\mathrm{F} 1-\mathrm{F} 5^{+}$and F7. Note that since $F_{k, V}$ is a concave function in $r$, it has first and second order derivatives almost everywhere in $\Gamma_{k, V}$ so that the differential inequalities in (1.4), (1.5), as well as condition $\mathrm{F} 5^{+}$, hold in this sense. We can also consider the case of infinite $V$ and replace $F_{k}$ by other functions. The resulting Bellman type augmented Hessian operators can then be treated by smooth approximation as in the $k=1$ case, (see for example [7]); and we would obtain the existence of $C^{2, \alpha}(\bar{\Omega})$ solutions, for some $\alpha>0$ in Theorems 1.4 and 4.1. More generally if we drop the smoothness condition $F \in C^{2}(\Gamma)$, then we still obtain existence of $C^{2, \alpha}(\bar{\Omega})$ solutions, for some $\alpha>0$, in Theorems 1.4, 4.1 and Remarks $4.1,4.2$. Here we need the more general $C^{2, \alpha}(\bar{\Omega})$ estimate for concave fully nonlinear uniformly elliptic equations from [35]; see also [15]. 
Examples for A. Examples of strictly regular matrix functions arising in optimal transportation and geometric optics can be found for example in $[11,28,31,42,45]$. Typically there is not a natural association with oblique boundary operators, except for those coming from the second boundary value problem to prescribe the images of the associated mappings, so that second derivative estimates may depend on gradient restrictions in accordance with Remark 1.2. Moreover the relevant equations typically involve constraints so that we are also in the situation of Remark 1.3. Both these situations will be further examined in ensuing work. However we will give some examples satisfying our hypotheses, where oblique boundary operators arise naturally through our domain convexity conditions.

Our first examples extend those coming from the conformal deformations of manifolds with boundary; (as for example in $[3,19,22]$ ). We introduce a class of matrix functions of the form

$$
A(x, z, p)=\frac{1}{2} a_{k l}(x, z) p_{k} p_{l} I-a_{0}(x, z) p \otimes p,
$$

where $a_{k l}, a_{0} \in C^{2}(\bar{\Omega} \times \mathbb{R})$ and the matrix $\left\{a_{k l}\right\}>0$ in $\bar{\Omega} \times \mathbb{R}$. Clearly for any vectors $\xi, \eta \in \mathbb{R}^{n}$, we have

$$
\begin{aligned}
A_{i j}^{k l} \xi_{i} \xi_{j} \eta_{k} \eta_{l} & =\left(a_{s t} \delta_{s k} \delta_{t l} \delta_{i j}-2 a_{0} \delta_{i k} \delta_{j l}\right) \xi_{i} \xi_{j} \eta_{k} \eta_{l} \\
& =|\xi|^{2} a_{k l} \eta_{k} \eta_{l}-2 a_{0}(\xi \cdot \eta)^{2} \\
& \geq \lambda_{1}|\xi|^{2}|\eta|^{2}-2 a_{0}(\xi \cdot \eta)^{2}
\end{aligned}
$$

where $\lambda_{1}>0$ denotes the minimum eigenvalue of $\left\{a_{k l}\right\}$, so that $A$ is strictly regular in $\bar{\Omega}$. Moreover $A$ is uniformly regular, with (1.21) satisfied with $\lambda_{0}=\inf \lambda_{1}, \bar{\lambda}_{0}=$ $2 \sup a_{0}^{+}$, where the infimum and supremum are taken over $\Omega \times(-M, M)$. For $A$ given by (4.15), the corresponding $A$-curvature matrix on $\partial \Omega$ for $(1.15)$ is given by

$$
K_{A}[\partial \Omega](x, z, p)=-\delta v(x)+a_{k l}(x, z) p_{k} v_{l}(x)(I-v(x) \otimes v(x)),
$$

where $v$ is the unit inner normal to $\partial \Omega$ and $\delta$ denotes the tangential gradient. Consequently the quasilinear boundary operator $\mathcal{G}$, given by (1.17) with $\beta_{k}=a_{k l} v_{l}$, will be oblique, satisfying $\beta \cdot v \geq \lambda_{0}$ on $\partial \Omega$ and $\Omega$ is uniformly $(\Gamma, A, G)$-convex with respect to $u$ if and only if

$$
-\delta v+\varphi(\cdot, u)(I-v \otimes v)+\mu_{0} v(x) \otimes v(x) \in \Gamma,
$$

for some constant $\mu_{0}>0$, possibly depending on $u$. Accordingly our uniform convexity condition is independent of the gradient variables. In the orthogonally invariant case, letting

$$
\tilde{\Gamma}=\lambda(\Gamma)=\left\{\lambda \in \mathbb{R}^{n} \mid \lambda_{1}, \ldots, \lambda_{n} \text { are eigenvalues of some } r \in \Gamma\right\}
$$

denote the corresponding cone to $\Gamma$ in $\mathbb{R}^{n},(4.18)$ is equivalent to $\left(\tilde{\kappa}, \mu_{0}\right) \in \tilde{\Gamma}$, where $\tilde{\kappa}_{i}=\kappa_{i}+\varphi, i=1, \ldots, n-1$, and $\kappa=\left(\kappa_{1}, \ldots, \kappa_{n-1}\right)$ denotes the principal curvatures 
of $\partial \Omega$. In particular for the cones $\Gamma_{k},(4.18)$ is equivalent to $\tilde{\kappa} \in \tilde{\Gamma}_{k-1}$, that is $S_{j}(\tilde{\kappa})>0$ for $1 \leq j \leq k-1$.

Conformal geometry. The application to conformal geometry concerns the special case $a_{i j}=\delta_{i j}, a_{0}=1$ in (4.15), that is

$$
A(p)=\frac{1}{2}|p|^{2} I-p \otimes p
$$

with the associated semilinear Neumann condition,

$$
D_{\nu} u=\varphi(x, u), \quad \text { on } \partial \Omega,
$$

and is related to the fully nonlinear Yamabe problem with boundary, where $A_{\tilde{g}}=$ $e^{2 u} M[u]$ is the Schouten tensor of the conformal deformation $\tilde{g}=e^{-2 u} g_{0}$ and $g_{0}$ denotes the standard metric on $\mathbb{R}^{n}$. If $F$ is positive homogenous of degree one, satisfying F3 with $a_{0}=0, \tilde{\varphi}$ is a positive function on $\Omega$ and $\tilde{h}$ a function on $\partial \Omega$, then the problem of finding a conformal metric $\tilde{g}$ on $\Omega$ such that $F\left(A_{\tilde{g}}\right)=\tilde{\varphi}$, with mean curvature $\tilde{h}$ on $\partial \Omega$, is equivalent to solving the semilinear Neumann problem,

$$
\begin{array}{cl}
\mathcal{F}[u]:=F(M[u])=\tilde{\varphi} e^{-2 u}, & \text { in } \Omega, \\
D_{\nu} u=\tilde{h} e^{-u}-h_{0}, & \text { on } \partial \Omega,
\end{array}
$$

where $h_{0}$ denotes the mean curvature of $\partial \Omega$ with respect to $g_{0}$. With $\Omega, \tilde{\varphi}$ and $\tilde{h}$ sufficiently smooth, (4.21) satisfies the hypotheses of the second derivative estimate, Theorem 1.2, if $F$ also satisfies F1 and F2 and $\Omega$ satisfies (4.18) with $\varphi=\tilde{h} e^{-u}-h_{0}$. Note that our restriction $r \leq \operatorname{trace}(r) I$ on $\Gamma$ implies that $\tilde{h}>0$. However (4.18) does provide some relaxation of the umbilic condition in [19] and related papers, possibly depending on solution upper bounds, and can be extended to more general Riemannian manifolds with boundary, (taking account of Remarks 2.1 and 3.6), as well as to more general boundary curvatures [43]. In particular for the cones, $\Gamma_{2}$ and $\mathcal{P}_{n-1}$, the convexity condition (4.18) is equivalent to $\tilde{h}>0$, since $\delta \cdot v=(1-n) h_{0}$ so no geometric conditions are needed. Note that for the local gradient bound, Theorem 3.1, we only need $F$ to satisfy F1 to fulfil the hypotheses of case (i) (and no geometric restrictions on $\Omega$ ). Such bounds are already established for the special case (4.19) in $[3,19]$. Since the functions $B$ and $\varphi$ are not monotone increasing in $z$ we would need though a priori solution bounds to get existence and this is still an unresolved issue in the non-umbilic case; (see [23] for recent $L^{\infty}$ estimates in the umbilic case).

Optimal transportation and geometric optics. In optimal transportation problems, the matrix $A$ is generated by a cost function $c \in C^{2}(\mathcal{D})$, where $\mathcal{D}$ is a domain in $\mathbb{R}^{n} \times \mathbb{R}^{n}$, through the relation

$$
A(x, p)=c_{x x}(x, Y(x, p))
$$


where the mapping $Y \in C^{1}(\mathcal{U})$, for some domain $\mathcal{U} \subset \mathbb{R}^{n} \times \mathbb{R}^{n}$, is given as the unique solution of

$$
c_{x}(x, Y)=p .
$$

Here we assume conditions A1, A2 as in [31,45] to guarantee the unique solvability of $Y$ from (4.23). The strict regularity was introduced as condition A3 in [31]; (see also [40]). More generally the matrices $A$ arise from prescribed Jacobian equations [41] where now the mapping $Y \in C^{1}(\mathcal{U})$ is given for a domain $\mathcal{U} \subset \mathbb{R}^{n} \times \mathbb{R} \times \mathbb{R}^{n}$ satisfying $\operatorname{det} Y_{p} \neq 0 \operatorname{in} \mathcal{U}$ and the matrix $A$ is given by

$$
A(x, z, p)=Y_{p}^{-1}\left(Y_{x}+Y_{z} \otimes p\right)
$$

Mappings $Y$ in geometric optics can also be unified through a notion of generating function [42], which extends that of a cost function to permit the $z$ dependence in $Y$ and provides symmetric matrices in (4.24). For further information and particular examples of strictly regular matrices $A$, the reader is referred to $[11,28,31,42,45]$ and the references therein. As mentioned above in most of these examples there are not natural relationships with semilinear oblique boundary operators so that the situation in Remarks 1.2 and 1.3 is applicable. The natural boundary condition is the prescription of the image $\Omega^{*}$ of the mapping $T:=Y(\cdot, u, D u)$ on $\Omega$, which implies a boundary condition which is oblique with respect to admissible functions $[41,45]$. Once the obliqueness is estimated we are in the situation of Theorem 1.2 and moreover our domain convexity conditions there originate from those used in the optimal transportation and more generally; (see [28,41,45]).

Accordingly we just mention here some examples which fit simply with our hypotheses. First the logarithm cost function, given by $c(x, y)=\frac{1}{2} \log |x-y|$ for $x \neq y$, also generates our example (4.19), [45]. From geometric optics we have the example coming from the reflection of a parallel beam to a flat target $[28,42]$,

$$
A(x, z, p)=\frac{1}{2 z}\left(|p|^{2}-1\right) I
$$

for $z>0$. Here there is a constraint, namely $u>0$, which is readily handled by taking a logarithm or assuming the subsolution $\underline{u}>0$ in $\bar{\Omega}$. Then for a semilinear Neumann boundary condition of the form

$$
D_{\nu} u=u \varphi(\cdot, u)
$$

we obtain again that $\Omega$ is uniformly $(\Gamma, A, G)$-convex with respect to $u$ if and only if (4.18) holds.

Admissible functions. Quadratic functions of the form $u_{0}=c_{0}+\frac{1}{2} \epsilon\left|x-x_{0}\right|^{2}$, will be admissible for the matrices (4.15) and for arbitrary constants $c_{0}$, points $x_{0} \in \Omega$ and 
sufficiently small $\epsilon$. In general for matrices $A$ arising in optimal transportation and geometric optics the existence of admissible functions is proved in [11].

Nonlinear boundary operators. The capillarity type operators, given by

$$
G(x, z, p)=p \cdot v-\theta(x) \sqrt{1+|p|^{2}}-\varphi(x, z),
$$

would satisfy our hypotheses for $0<\theta<1$ on $\partial \Omega$. Furthermore for $A$ in the form (4.15) with $\left\{a_{i j}\right\}=I$, condition (4.18) would at least imply that that $\Omega$ is uniformly ( $\Gamma, A, G$ )-convex with respect to $u$. Note that here and quite generally we cannot have $\varphi(\cdot, u) \geq 0$ everywhere on $\partial \Omega$ for an admissible function so the basic capillarity condition is ruled out by our concavity condition which requires $\theta>0$.

\subsection{Degenerate equations}

In this subsection, we consider the extension of our results to degenerate elliptic equations and in particular apply the classical existence results, Theorems 4.1 and 4.2 , to yield the existence of $C^{1,1}$ admissible solutions for the oblique boundary value problems. We shall use the following assumption, in place of $\mathrm{F} 1$, to describe the degenerate ellipticity:

F1 $^{-} F$ is non-decreasing in $\Gamma$, namely

$$
F_{r}:=F_{r_{i j}}=\left\{\frac{\partial F}{\partial r_{i j}}\right\} \geq 0, \text { in } \Gamma,
$$

and $\mathscr{T}(r):=\operatorname{trace}\left(F_{r}\right)>0$ in $\Gamma$.

Then for $\Gamma \subset \Gamma_{1}$, using an elliptic regularisation as in [37], we define for a constant $\epsilon \geq 0, F_{1}(r)=\operatorname{trace}(r)>0$, approximating operators and cones,

$$
F^{\epsilon}(r)=F\left(r+\epsilon F_{1}(r) I\right), \quad \Gamma^{\epsilon}=\left\{r \in \mathbb{S}^{n} \mid r+\epsilon F_{1}(r) I \in \Gamma\right\} .
$$

Clearly $F^{\epsilon}$ satisfies the ellipticity condition $\mathrm{F} 1$ in the cone $\Gamma^{\epsilon}$, for $\epsilon>0$ and is also uniformly elliptic there with

$$
\epsilon \mathscr{T}\left(r+\epsilon F_{1}(r) I\right) I \leq F_{r}^{\epsilon} \leq(1+\epsilon) \mathscr{T}\left(r+\epsilon F_{1}(r) I\right) I
$$

Moreover if $F$ also satisfies any of conditions $\mathrm{F} 2$ to $\mathrm{F}^{+}$or $\mathrm{F} 7$, then $F^{\epsilon}$ satisfies the same condition in $\Gamma^{\epsilon}$ with relevant constants independent of $\epsilon$, as $\epsilon$ tends to 0 . Consequently we may replace $F$ by $F^{\epsilon}$ and the operator $\mathcal{F}$ by $\mathcal{F}^{\epsilon}$, for sufficiently small $\epsilon \geq 0$ in the quasilinear boundary operator cases of our Hessian and gradient estimates in Sects. 2 and 3. To get the lower second derivative bounds in Theorem 1.1 we can simply use $\mathscr{T}^{\epsilon}(r):=\operatorname{trace}\left(F_{r}^{\epsilon}\right)>0$ in $\Gamma_{\epsilon}$, while for the lower tangential bounds in Theorem 1.2 we now have, from our restriction $\Gamma \in \mathcal{P}_{n-1}$ in the quasilinear case, 


$$
M[u] \leq(1+n \epsilon) \mathcal{F}_{1}[u] I,
$$

so that we arrive again at an estimate of the form (2.64). Note that we only need sufficiently small $\epsilon$ for the quasilinear case of Theorem 1.2. Clearly we could have assumed the weaker condition $\mathrm{F}^{-}$at the outset for our derivative estimates in Sects. 2 and 3 but it is not feasible then to consider solutions with smooth second derivatives. By approximation we now obtain from Theorem 4.1 the following extension to $C^{1,1}(\bar{\Omega})$ solvability of degenerate equations. Here a function $u \in C^{1,1}(\Omega)$ is admissible if $M[u] \in \Gamma$ almost everywhere in $\Omega$ and is a solution of Eq. (1.1) if it is a solution almost everywhere in $\Omega$.

Corollary 4.1 In the hypotheses of Theorem 4.1 assume that condition $F 1$ is weakened to condition $F^{-}, \partial \Omega \in C^{3}, \beta \in C^{2}(\partial \Omega), \varphi \in C^{2}(\partial \Omega \times \mathbb{R})$ with the subsolution and supersolution conditions strengthened so that at least one of the inequalities (1.25) holds strictly in $\Omega$. Then there exists an admissible solution $u \in C^{1,1}(\bar{\Omega})$ of the boundary value problem, (1.1)-(1.2).

Proof Assume first that $\mathcal{F}[\bar{u}]<B(\cdot, u, D u)$ in $\Omega$ so that assuming, initially that $\underline{u}, \bar{u} \in C^{2}(\bar{\Omega})$, (as in the proof of Theorem 4.1), $\bar{u}$ will be a supersolution of (1.1)(1.2), with $B$ replaced by $B-\delta$, for some sufficiently small positive constant $\delta$. We claim then that $\bar{u}$ and $\underline{u}$ are respectively supersolution and admissible subsolution of the boundary value problem (1.1)-(1.2) for $\mathcal{F}$ replaced by $\mathcal{F}^{\epsilon}$ for sufficiently small $\epsilon$, depending on $\bar{u}$ and $\delta$. To prove this we first define the sets

$$
\Omega_{\epsilon}^{\prime}=\left\{x \in \bar{\Omega} \mid M[\bar{u}](x)+\epsilon F_{1}(M[\bar{u}](x)) I \in \Gamma_{\epsilon}\right\}, \quad K_{\epsilon}=\left\{x \in \bar{\Omega}_{\epsilon}^{\prime} \mid \mathcal{F}^{\epsilon}[\bar{u}] \geq a\right\},
$$

where $a$ is constant satisfying $a_{0}<a<B(\cdot, \bar{u}, D \bar{u})$ in $\Omega$. Then $K_{\epsilon}$ is a decreasing family of compact subsets of $\bar{\Omega}$ approaching $K_{0}$ as $\epsilon$ approaches zero. Consequently $K_{\epsilon} \subset \Omega_{0}^{\prime}$ for sufficiently small $\epsilon$. By the concavity F2, (and assuming initially $\underline{u} \in$ $C^{2}(\bar{\Omega})$ as in the proof of Theorem 4.1), we then have, in $K_{\epsilon}$,

$$
\mathcal{F}^{\epsilon}[\bar{u}] \leq \mathcal{F}[\bar{u}]+\epsilon \mathcal{F}_{1}[\bar{u}] \mathscr{T}(M[\bar{u}]) \leq B(\cdot, \bar{u}, D \bar{u})
$$

for sufficiently small $\epsilon$ depending on $\bar{u}$ and $\delta$. Clearly $\mathcal{F}^{\epsilon}[\bar{u}] \leq B(\cdot, \bar{u}, D \bar{u})$ in $\Omega_{\epsilon}^{\prime}-K_{\epsilon}$ so that $\bar{u}$ is a supersolution of the equation, $\mathcal{F}^{\epsilon}=B$, for sufficiently small $\epsilon$. Next it follows immediately from the degenerate ellipticity $\mathrm{F} 1^{-}$, that $\underline{u}$ is an admissible subsolution, for any $\epsilon \geq 0$, so that our claim is proved.

From Theorem 4.1, (Remark 4.2), and Theorems 1.1, 1.2 and 1.3, there exists a unique solution $u_{\epsilon} \in C^{2}(\bar{\Omega})$ of the problem (1.1)-(1.2) with $\mathcal{F}=\mathcal{F}^{\epsilon}$ for sufficiently small positive $\epsilon$, together with the a priori estimates

$$
\left|u_{\epsilon}\right|_{2 ; \Omega} \leq C
$$

with constant $C$ independent of $\epsilon$. Hence there exists a subsequence $u_{\epsilon_{k}}$ and a function $u \in C^{1,1}(\bar{\Omega})$ such that

$$
u_{\epsilon_{k}} \rightarrow u \quad \text { in } C^{1, \alpha}(\bar{\Omega}), \quad \forall \alpha \in(0,1), \quad \text { as } \epsilon_{k} \rightarrow 0 .
$$


From the stability property of viscosity solutions $[4,5]$, it is readily seen that $u \in$ $C^{1,1}(\bar{\Omega})$ is an admissible solution of the problem (1.1)-(1.2).

Finally in the case that $\underline{u}$ is a strict subsolution of (1.1), we replace $B$ by $B+\delta$ for sufficiently small positive $\delta$ and let $\delta \rightarrow 0$.

To illustrate the application of Corollary 4.1, we consider the degenerate elliptic operators $\mathfrak{M}_{k}$, given by functions

$$
\mathfrak{m}_{k}(r)=\min \left\{\sum_{s=1}^{k} \lambda_{i_{s}}(r)\right\},
$$

for $k=1, \ldots, n, i_{1}, \ldots, i_{k} \subset\{1, \ldots, n\}$, in the cones $\mathcal{P}_{k}$ introduced in (4.11). Namely, $\mathfrak{M}_{k}[u]:=\mathfrak{m}_{k}(M[u])$. As for the examples (4.14), the functions $\mathfrak{m}_{k}$ for $k<n$ are not $C^{2}$ but will still satisfy conditions F1 ${ }^{-}, \mathrm{F} 2, \mathrm{~F} 3, \mathrm{~F} 4, \mathrm{~F} 5$ and F7, with $a_{0}=0$, in $\mathcal{P}_{k}$ almost everywhere. As well $\mathfrak{m}_{k}$ is positive homogeneous of degree one. The functions $\mathfrak{m}_{k}$ are also related to our examples (4.13) since $\mathfrak{m}_{k}=F_{k,-\infty}=\lim _{\alpha \rightarrow \infty} F_{k,-\alpha}$. More explicitly the normalised functions $\tilde{F}_{k,-\alpha}:=\left(C_{n}^{k}\right)^{\frac{1}{\alpha}} F_{k,-\alpha}$ are monotone decreasing in $\alpha$ and satisfy the inequalities

$$
\mathfrak{m}_{k} \leq \tilde{F}_{k,-\alpha} \leq\left(C_{n}^{k}\right)^{\frac{1}{\alpha}} \mathfrak{m}_{k}
$$

in $\mathcal{P}_{k}$. We also note that when $k=n, \mathfrak{M}_{n}$ is the Poisson operator $\mathcal{F}_{1}$.

By suitable approximation of the "minimum" function we then obtain from Corollary 4.1 the following analogue of Theorem 1.4.

Corollary 4.2 Let $\mathcal{F}=\mathfrak{M}_{k}$, for some $k=1, \ldots, n-1, \Omega$ a bounded $C^{3}$ domain in $\mathbb{R}^{n}, A \in C^{2}\left(\bar{\Omega} \times \mathbb{R} \times \mathbb{R}^{n}\right)$ strictly regular in $\bar{\Omega}, B>0, \in C^{2}\left(\bar{\Omega} \times \mathbb{R} \times \mathbb{R}^{n}\right)$, $\mathcal{G}$ semilinear and oblique with $\beta \in C^{2}(\partial \Omega), \varphi \in C^{2}(\partial \Omega \times \mathbb{R})$ satisfying (1.23). Assume that $\underline{u}$ and $\bar{u}, \in C^{2}(\Omega) \cap C^{1}(\bar{\Omega})$ are respectively an admissible subsolution of (1.1)-(1.2) and supersolution of (1.1)-(1.2), with at least one of them strict, and $\Omega$ is uniformly $\left(\mathcal{P}_{k}, A, G\right)$-convex with respect to the interval $\mathcal{I}=[\underline{u}, \bar{u}]$. Assume also that $A, B$ and $\varphi$ are non-decreasing in $z$, with at least one of them strictly increasing, A satisfies the quadratic growth conditions (1.22) and $B$ is independent of $p$. Then if one of the following further conditions is satisfied:

(i) A is uniformly regular;

(ii) $\beta=v$ and $A=o\left(|p|^{2}\right)$ in (1.22);

(iii) $k=1$ and $A \geq O\left(|p|^{2}\right)$ I in place of (1.22),

there exists an admissible solution $u \in C^{1,1}(\bar{\Omega})$ of the boundary value problem (1.1)(1.2).

We remark that we may also prove Corollary 4.2 directly from Theorem 4.1 by approximating $\mathfrak{m}_{k}$ by $F_{k,-\alpha}$ for large $\alpha$. Furthermore the function $\mathfrak{m}_{1}$ satisfies condition F6 in $\mathbb{S}^{n}$ since, from its orthogonal invariance, we have

$$
\mathcal{E}_{2}=\left[\mathfrak{m}_{1}(r)\right]^{2} \leq \max \left\{a^{2}, b^{2}\right\} \leq o(|r|) \mathscr{T}, \quad \text { as }|r| \rightarrow \infty,
$$


for $r \in \mathbb{S}^{n}, a \leq \mathfrak{m}_{1}(r) \leq b$ and using $\mathscr{T}=1$. Clearly, $\mathfrak{m}_{1}$ satisfies F6 in the positive cone $K^{+}$. In this case we obtain, by approximation by $F_{1,-\alpha}$, from Theorem 4.2 the following existence of $C^{1,1}$ admissible solutions for the oblique boundary value problem (1.1)-(1.2) with $\mathcal{F}=\mathfrak{M}_{1}$ and nonlinear $\mathcal{G}$.

Corollary 4.3 Let $\mathcal{F}=\mathfrak{M}_{1}, \Omega$ a bounded $C^{3}$ domain in $\mathbb{R}^{n}, A \in C^{2}\left(\bar{\Omega} \times \mathbb{R} \times \mathbb{R}^{n}\right)$ strictly regular in $\bar{\Omega}, B>0, \in C^{2}\left(\bar{\Omega} \times \mathbb{R} \times \mathbb{R}^{n}\right), G \in C^{2}\left(\partial \Omega \times \mathbb{R} \times \mathbb{R}^{n}\right)$ is concave with respect to $p$ and uniformly oblique in the sense of (4.5). Assume that $\underline{u}$ and $\bar{u}, \in C^{2}(\Omega) \cap C^{1}(\bar{\Omega})$ are respectively an admissible subsolution of (1.1)-(1.2) and supersolution of (1.1)-(1.2) with at least one of them strict and $\Omega$ is uniformly $(A, G)$ convex with respect to the interval $\mathcal{I}=[\underline{u}, \bar{u}]$. Assume also that $A, B$ and $-G$ are non-decreasing in $z$, with at least one of them strictly increasing, A satisfies the quadratic growth conditions (3.75) and $B$ is independent of $p$. Then there exists an admissible solution $u \in C^{1,1}(\bar{\Omega})$ of the boundary value problem (1.1)-(1.2).

Proof First we note that the approximations $F^{\epsilon}$ defined in (4.29) do not satisfy F6 for $\epsilon>0$. Turning to the approximations $F_{1,-\alpha}$, with $\alpha \geq 1$, we have

$$
\mathcal{E}_{2}=\frac{\sum_{i=1}^{n}\left[\lambda_{i}(r)\right]^{1-\alpha}}{\sum_{i=1}^{n}\left[\lambda_{i}(r)\right]^{-1-\alpha}} \mathscr{T} \leq n\left[\mathfrak{m}_{1}(r)\right]^{2} \mathscr{T} \leq n^{1+\frac{2}{\alpha}}\left[F_{1,-\alpha}(r)\right]^{2} \mathscr{T},
$$

by (4.35), so that F6 is satisfied in $\Gamma_{n}$ for $F_{1,-\alpha}(r) \leq b<\infty$. Now we apply Theorem 4.2 to the normalised functions $\tilde{F}_{1,-\alpha}$ and as $\alpha \rightarrow \infty$, we conclude the existence of an admissible solution $u \in C^{1,1}(\bar{\Omega})$ of the boundary value problem (1.1)-(1.2), similarly to the proof of Corollary 4.1. For this we also observe that from the inequalities (4.35) we have automatically that $\underline{u}$ and $\bar{u}$ are respectively an admissible subsolution and supersolution of (1.1)-(1.2), with $F=\tilde{F}_{1,-\alpha}$, for $\left(n^{\frac{1}{\alpha}}-\right.$ 1) $\mathfrak{m}_{1}(M[\bar{u}]) \leq \delta$.

Note that if we use the functions $F_{1,-\alpha}$, instead of their normalisations, in the proof of Corollary 4.3 we can cover the case that $\underline{u}$ is an admissible strict subsolution of (1.1)-(1.2), without having to modify $B$. Using the approximations $F_{k,-\alpha}$ in the proof of Corollary 4.2, we can similarly adjust the proof of Corollary 4.2.

We remark that the functions $F_{1,-\alpha}$, with $0<\alpha<1$, also satisfy F6 in $\Gamma_{n}$ for $F_{1,-\alpha}(r) \leq b<\infty$, since by a simple modification of (4.37) we have

$$
\mathcal{E}_{2} \leq n|r|^{1-\alpha}\left[\mathfrak{m}_{1}(r)\right]^{1+\alpha} \mathscr{T} \leq n^{2+\frac{1}{\alpha}}|r|^{1-\alpha}\left[F_{1,-\alpha}(r)\right]^{1+\alpha} \mathscr{T} \leq o(|r|) \mathscr{T},
$$

as $|r| \rightarrow \infty$.

Also the solutions in Corollaries 4.1, 4.2 and 4.3 will be unique if either $A$ or $B$ are strictly increasing, in which case the strictness of the subsolution or supersolution condition is not explicitly needed in their hypotheses. When only $\varphi$ or $-G$ is strictly increasing we need to utilize appropriate barrier constructions, as considered in Section 2 of [13], to prove uniqueness. In particular if $\underline{u} \in C^{2}(\bar{\Omega})$ is a strict admissible subsolution of (1.1), the uniqueness, along with the more general comparison principles, follows from Part (ii) of Lemma 2.1 in [13]. 
To conclude this section we also point out that our estimates and techniques also embrace degenerate elliptic equations where $B \geq a_{0}>-\infty$, with weakly admissible solutions $u \in C^{1,1}(\Omega)$ now satisfying $M[u] \in \bar{\Gamma}$, a.e. in $\Omega$, using property F5 with $a=a_{0}$, (which as shown in Sect. 4.2 is also a consequence of conditions $\mathrm{F}^{-}$, $\mathrm{F} 2$ and F3). As remarked in Sect. 1, we can also assume $a_{0}=0$ in this case and, as shown there, concave one-homogeneous functions $F$ will be typical examples. In particular the constants $C$ in the estimates (1.14), (1.18), (1.24), (2.23), (2.49) and (3.55) will not depend on a lower bound for $B(\cdot, u, D u)$, (provided in the cases where $\mathrm{F}^{+}$is assumed, we also assume $\left.a=a_{0}>-\infty\right)$. Our resultant existence results, Corollaries 4.1, 4.2 and 4.3 now extend similarly, under the strictness of the subsolution $\underline{u}$, in their hypotheses and as above, the resultant $C^{1,1}$ solutions are unique. Note that in the totally degenerate case, $B=0$ in $\Omega$, an admissible function will furnish a suitable strict subsolution, (after subtraction of a sufficiently large constant), when $-G$ is strictly increasing. More details of these arguments, as well as extensions to $\underline{u}$ and $\bar{u} \in C^{1,1}$, will be provided in conjunction with our treatment of weak solutions in [15].

\subsection{Final remarks}

The oblique boundary value problem (1.1)-(1.2) for augmented Hessian equations is natural in the classical theory of fully nonlinear elliptic equations. In this paper and its sequel [13], we have treated this problem in a very general setting. Through a priori estimates, we have established the classical existence theorems under appropriate domain convexity hypotheses for both (i) strictly regular $A$ and semilinear or concave $\mathcal{G}$, and (ii) regular $A$ and uniformly concave $\mathcal{G}$. Our emphasis in this paper is the case (i), since the case (ii) is already known in the context of the second boundary value problems of Monge-Ampère equations $[49,50]$ and optimal transportation equations $[45,51]$. In case (i), the boundary conditions can be any oblique conditions, including the special case of the Neumann problem, while the operators embrace a large class including the Monge-Ampère operator, $k$-Hessian operators and their quotients, as well as degenerate and non-orthogonally invariant operators.

In part II [13] we treat the case of regular matrices $A$ which includes the basic Hessian equation case, where $A=0$ or more generally where $A$ is independent of the gradient variables. A fundamental tool here is the extension of our barrier constructions for Monge-Ampère operators in $[11,17]$ to general operators; (see Remarks in Section 2 of [16]). In general as indicated by the Pogorelov example [47,52], we cannot expect second derivative estimates for arbitrary linear oblique boundary conditions and moreover the strict regularity of $A$ is critical for our second derivative estimates in Sect. 2. We remark though that our methods in this paper, as further developed in [13], also show that the strict regularity can be replaced by not so natural, strong monotonicity conditions with respect to the solution variable on either the matrix $A$ or the boundary function $\varphi$, that is either $A_{z}$ or $\varphi_{z}$ is sufficiently large, and the latter would include the case when $A=0$, in agreement with the Monge-Ampère case in $[46,47,52]$. For Monge-Ampère type operators, we are able to derive the second derivative bound for semilinear Neumann boundary value problem when $A$ is just 
regular, under additional assumption of the existence of an admissible supersolution $\bar{u}$, satisfying $\operatorname{det}(M[\bar{u}]) \leq B(\cdot, \bar{u}, D \bar{u})$ in $\Omega$ and $D_{v} \bar{u}=\varphi(\cdot, \bar{u})$ on $\partial \Omega$; (see Jiang et al. [16]). This is an extension of the fundamental result in [27] for the standard Monge-Ampère operator, (although the supersolution hypothesis is not needed in [27] and more generally when $D_{p x} A=0$ and $D_{p z} A=0$ [16]). For the semilinear oblique problem for standard $k$-Hessian equations, the known results due to Trudinger [36] and Urbas [47], where the second derivative estimates for Neumann problem in balls, and for oblique problem in general domains in dimension two respectively were studied. Recently the Neumann problem for the standard $k$-Hessian equation has been studied in uniformly convex domains in [29]. However, it would be reasonable to expect there are corresponding second derivative estimates for admissible solutions of the Neumann problem for $k$-Hessian equations in uniformly $(k-1)$-convex domains. More generally, the second derivative estimate for admissible solutions of the Neumann problem of the augmented $k$-Hessian equations, with only regular $A$, in uniformly $\left(\Gamma_{k}, A, G\right)$-convex domains is still an open problem.

In Sect. 3, we have established the gradient estimate for augmented Hessian equations in the cones $\Gamma_{k}$ when $k>n / 2$ under structure conditions for $A$ and $B$ corresponding to the natural conditions of Ladyzhenskaya and Ural'tseva for quasilinear elliptic equations [7,21]. The gradient estimate under natural conditions is also known for $k=1$ and is a special case of the uniformly elliptic case [7,25]. Therefore, it would be interesting to prove gradient estimates, (interior and global), for both oblique and Dirichlet boundary value problems under natural conditions for the remaining cases for operators in the cones $\Gamma_{k}$ when $2 \leq k \leq n / 2$, and in particular for the basic Hessian operators $F_{k}$ when $2 \leq k \leq n / 2$, which also enjoy $L^{p}$ gradient estimates for $p<n k /(n-k)$ [44]. In [14], we apply our gradient estimates here and general barrier constructions in Section 2 of [13] to study the classical Dirichlet problem for general augmented Hessian equations with only regular matrix functions $A$. Here as well as our conditions on $F$ in case (ii) of Theorem 1.1, for global second derivative estimates we also need to assume orthogonal invariance and the existence of an appropriate subsolution, as in our previous papers $[17,18]$. Our barrier constructions in [13] also permit some relaxation of the conditions on $F$ in the regular case, as already indicated in Remark 3.2.

As pointed out in Remark 1.2, our domain convexity conditions require some relationship between the matrix $A$ and the boundary operator $\mathcal{G}$. If we drop these from our hypotheses, we can still infer the existence of classical solutions of the Eq. (1.1) which are globally Lipschitz continuous and satisfy the boundary condition (1.2) in a weak viscosity sense [4] so that our domain convexity conditions become conditions for boundary regularity. This situation is further amplified in [15], with extensions as well to strong solutions in the degenerate cases. Further investigations involve the examination of the sharpness of our boundary convexity conditions for global second derivative bounds. A preliminary result here for the conformal geometry application is already given in [24]. When these degenerate we would still expect to have regularity in the umbilic case, as in $[3,19]$, which would embrace for example the case $\tilde{h}=0$ in (4.21).

Note As indicated above, this paper is the first in a series of papers on oblique boundary value problems for augmented Hessian equations and its original version was posted in 
late 2015 [12]. Its immediate sequel [13] and their application to the Dirichlet problem [14] were originally planned in 2015 as part of the present paper but separated in order not to delay for too long the propagation of the main result, Theorem 1.2 and its ramifications.

Open Access This article is distributed under the terms of the Creative Commons Attribution 4.0 International License (http://creativecommons.org/licenses/by/4.0/), which permits unrestricted use, distribution, and reproduction in any medium, provided you give appropriate credit to the original author(s) and the source, provide a link to the Creative Commons license, and indicate if changes were made.

\section{References}

1. Caffarelli, L., Nirenberg, L., Spruck, J.: The Dirichlet problem for nonlinear second-order elliptic equations III: functions of eigenvalues of the Hessians. Acta Math. 155, 261-301 (1985)

2. Caffarelli, L., Nirenberg, L., Spruck, J.: Nonlinear second-order elliptic equations V. The Dirichlet problem for Weingarten hypersurfaces. Commun. Pure Appl. Math. 41, 47-70 (1988)

3. Chen, S.-Y.S.: Boundary value problems for some fully nonlinear elliptic equations. Calc. Var. Partial Differ. Equ. 30(1), 1-15 (2007)

4. Crandall, M.G., Ishii, H., Lions, P.L.: User's guide to viscosity solutions of second order partial differential equations. Bull. Am. Math. Soc. 27(1), 1-67 (1992)

5. Evans, L.C.: A convergence theorem for solutions of nonlinear second-order elliptic equations. Indiana Univ. Math. J. 27, 875-887 (1978)

6. Gerhardt, G.: Closed Weingarten hypersurfaces in Riemannian manifolds. J. Differ. Geom. 43, 612-641 (1996)

7. Gilbarg, D., Trudinger, N.S.: Elliptic Partial Differential Equations of the Second Order, 2nd edn. Springer, Berlin (1983). (reprinted 2001)

8. Guan, B.: Second order estimates and regularity for fully nonlinear elliptic equations on Riemannian manifolds. Duke Math. J. 163, 1491-1524 (2014)

9. Ivochkina, N.: Solution of the Dirichlet problem for some equations of Monge-Ampère type. Mat. Sb. 128, 403-415 (1985). (English translation in Math. USSR-Sb. 56(403-415), (1987))

10. Ivochkina, N., Trudinger, N.S., Wang, X.-J.: The Dirichlet problem for degenerate Hessian equations. Commun. Partial Differ. Equ. 26, 859-882 (2004)

11. Jiang, F., Trudinger, N.S.: On Pogorelov estimates in optimal transportation and geometric optics. Bull. Math. Sci. 4, 407-431 (2014)

12. Jiang, F., Trudinger, N.S.: Oblique Boundary Value Problems for Augmented Hessian Equations I, preprint (2015). arXiv:1511.08935

13. Jiang, F., Trudinger, N.S.: Oblique boundary value problems for augmented Hessian equations II. Nonlinear Anal. 154, 148-173 (2017)

14. Jiang, F., Trudinger, N.S.: On the Dirichlet problem for general augmented Hessian equations, preprint (2017)

15. Jiang, F., Trudinger, N.S.: Oblique boundary value problems for augmented Hessian equations III, preprint (2018)

16. Jiang, F., Trudinger, N.S., Xiang, N.: On the Neumann problem for Monge-Ampère type equations. Can. J. Math. 68, 1334-1361 (2016)

17. Jiang, F., Trudinger, N.S., Yang, X.-P.: On the Dirichlet problem for Monge-Ampère type equations. Calc. Var. Partial Differ. Equ. 49, 1223-1236 (2014)

18. Jiang, F., Trudinger, N.S., Yang, X.-P.: On the Dirichlet problem for a class of augmented Hessian equations. J. Differ. Equ. 258, 1548-1576 (2015)

19. Jin, Q., Li, A., Li, Y.Y.: Estimates and existence results for a fully nonlinear Yamabe problem on manifolds with boundary. Calc. Var. Partial Differ. Equ. 28, 509-543 (2007)

20. Krylov, N.V.: On the general notion of fully nonlinear second-order elliptic equations. Trans. Am. Math. Soc. 347, 857-895 (1995)

21. Ladyzhenskaya, O., Ural'tseva, N.: Linear and Quasilinear Elliptic Equations. Academic Press, New York (1968) 
22. Li, A., Li, Y.Y.: A fully nonlinear version of the Yamabe problem on manifolds with boundary. J. Eur. Math. Soc. 8(2), 295-316 (2006)

23. Li, Y.Y., Nguyen, L.: A fully nonlinear version of the Yamabe problem on locally conformally flat manifolds with umbilic boundary. Adv. Math. 251, 87-110 (2014)

24. Li, Y.Y., Nguyen, L.: Counterexamples to $C^{2}$ boundary estimates for a fully nonlinear Yamabe problem on manifolds with boundary. Adv. Nonlinear Stud. 12, 783-797 (2012)

25. Lieberman, G.M., Trudinger, N.S.: Nonlinear oblique boundary value problems for nonlinear elliptic equations. Trans. Am. Math. Soc. 295, 509-546 (1986)

26. Lions, P.-L., Trudinger, N.S.: Linear oblique derivative problems for the uniformly elliptic HamiltonJacobi-Bellman equation. Math. Z. 191, 1-15 (1986)

27. Lions, P.-L., Trudinger, N.S., Urbas, J.: The Neumann problem for equations of Monge-Ampère type. Commun. Pure Appl. Math. 39, 539-563 (1986)

28. Liu, J., Trudinger, N.S.: On classical solutions of near field reflection problems. Discrete Cont. Dyn. Syst. 36(2), 895-916 (2016)

29. Ma, X.-N., Qiu, G.: The Neumann Problem for Hessian Equations, preprint (2015). arXiv:1508.00196

30. Ma, X.-N., Qiu, G., Xu, J.: Gradient estimates on Hessian equations for Neumann problem. Sci. Sin. Math. (Chinese) 46(8), 1117-1126 (2016)

31. Ma, X.-N., Trudinger, N.S., Wang, X.-J.: Regularity of potential functions of the optimal transportation problem. Arch. Ration. Mech. Anal. 177, 151-183 (2005)

32. Pogorelov, A.V.: The Minkowski Multidimensional Problem. Wiley, New York (1978)

33. Sha, J.-P.: p-convex Riemannian manifolds. Invent. Math. 83, 437-447 (1986)

34. Sha, J.-P.: Handlebodies and p-convexity. J. Differ. Geom. 25, 353-361 (1987)

35. Trudinger, N.S.: Boundary value problem for fully nonlinear elliptic equations. Proc. Centre Math. Anal. Aust. Natl. Univ. 8, 65-83 (1984)

36. Trudinger, N.S.: On degenerate fully nonlinear elliptic equations in balls. Bull. Aust. Math. Soc. 35, 299-307 (1987)

37. Trudinger, N.S.: The Dirichlet problem for the prescribed curvature equations. Arch. Ration. Mech. Anal. 111, 153-179 (1990)

38. Trudinger, N.S.: On the Dirichlet problem for Hessian equations. Acta Math. 175, 151-164 (1995)

39. Trudinger, N.S.: Weak solutions of Hessian equations. Commun. Partial Differ. Equ. 22, 1251-1261 (1997)

40. Trudinger, N.S.: Recent developments in elliptic partial differential equations of Monge-Ampère type. Proc. Int. Cong. Math. Madrid 3, 291-302 (2006)

41. Trudinger, N.S.: On the prescribed Jacobian equation, Gakuto Intl. Series, Math. Sci. Appl. 20, Proc. Intl. Conf. for the 25th Anniversary of Viscosity Solutions, 243-255 (2008)

42. Trudinger, N.S.: On the local theory of prescribed Jacobian equations. Discrete Cont. Dyn. Syst. 34(4), 1663-1681 (2014)

43. Trudinger, N.S.: From optimal transportation to conformal geometry, Conference talks (2016-2018)

44. Trudinger, N.S., Wang, X.-J.: Hessian measures II. Ann. Math. 150, 579-604 (1999)

45. Trudinger, N.S., Wang, X.-J.: On the second boundary value problem for Monge-Ampère type equations and optimal transportation. Ann. Scuola Norm. Sup. Pisa Cl. Sci VIII, 143-174 (2009)

46. Urbas, J.: The oblique derivative problem for equations of Monge-Ampère type in two dimensions. Proc. Centre Math. Anal. Aust. Natl. Univ. 12, 171-195 (1987)

47. Urbas, J.: Nonlinear oblique boundary value problems for Hessian equations in two dimensions. Ann. Inst. Henri Poincare-Analyse Non Linear 12, 507-575 (1995)

48. Urbas, J.: On the second boundary value problem for equations of Monge-Ampère type. J. Reine angew. Math. 487, 115-124 (1997)

49. Urbas, J.: Oblique boundary value problems for equations of Monge-Ampère type. Calc. Var. Partial Differ. Equ. 7, 19-39 (1998)

50. Urbas, J.: The second boundary value problem for a class of Hessian equations. Commun. Partial Differ. Equ. 26, 859-882 (2001)

51. von Nessi, G.T.: On the second boundary value problem for a class of modified-Hessian equations. Commun. Partial Differ. Equ. 35, 745-785 (2010)

52. Wang, X.-J.: Oblique derivative problems for the equations of Monge-Ampère type. Chin. J. Contemp. Math. 13, 13-22 (1992) 
Publisher's Note Springer Nature remains neutral with regard to jurisdictional claims in published maps and institutional affiliations. 